\title{
The Galactic WN stars
}

\section{Spectral analyses with line-blanketed model atmospheres versus stellar evolution models with and without rotation ${ }^{\star}$}

\author{
W.-R. Hamann, G. Gräfener, and A. Liermann
}

\begin{abstract}
Lehrstuhl Astrophysik der Universität Potsdam, Am Neuen Palais 10, 14469 Potsdam, Germany e-mail: wrh@astro.physik.uni-potsdam.de
\end{abstract}

Received 20 February 2006 / Accepted 6 June 2006

\begin{abstract}
Context. Very massive stars pass through the Wolf-Rayet (WR) stage before they finally explode. Details of their evolution have not yet been safely established, and their physics are not well understood. Their spectral analysis requires adequate model atmospheres, which have been developed step by step during the past decades and account in their recent version for line blanketing by the millions of lines from iron and iron-group elements. However, only very few WN stars have been re-analyzed by means of line-blanketed models yet.

Aims. The quantitative spectral analysis of a large sample of Galactic WN stars with the most advanced generation of model atmospheres should provide an empirical basis for various studies about the origin, evolution, and physics of the Wolf-Rayet stars and their powerful winds.

Methods. We analyze a large sample of Galactic WN stars by means of the Potsdam Wolf-Rayet (PoWR) model atmospheres, which account for iron line blanketing and clumping. The results are compared with a synthetic population, generated from the Geneva tracks for massive star evolution.

Results. We obtain a homogeneous set of stellar and atmospheric parameters for the Galactic WN stars, partly revising earlier results. Conclusions. Comparing the results of our spectral analyses of the Galactic WN stars with the predictions of the Geneva evolutionary calculations, we conclude that there is rough qualitative agreement. However, the quantitative discrepancies are still severe, and there is no preference for the tracks that account for the effects of rotation. It seems that the evolution of massive stars is still not satisfactorily understood.
\end{abstract}

Key words. stars: mass-loss - stars: winds, outflows - stars: Wolf-Rayet - stars: atmospheres - stars: early-type - stars: evolution

\section{Introduction}

Very massive stars pass through the Wolf-Rayet (WR) stage before they finally explode as supernovae or, possibly, $\gamma$-ray bursts. The WR stars are important sources of ionizing photons, momentum, and chemical elements. However, the evolution of massive stars has not yet been safely established, and their physics is not well understood.

The empirical knowledge about WR stars is hampered by difficulties with their spectral analysis. Adequate model atmospheres, which account for the non-LTE physics and their supersonic expansion, have been developed step by step during the past decades. In a previous paper we presented a comprehensive analysis of the Galactic WN stars from their helium, hydrogen, and nitrogen spectra (Hamann \& Koesterke 1998, hereafter quoted as Paper I). The major deficiency of this generation of model atmospheres was its neglect of line blanketing by the millions of lines from iron and iron-group elements. Hillier \& Miller (1998) were the first to include this important effect in their code CMFGEN. The line-blanketed version of the Potsdam Wolf-Rayet (PoWR) model atmosphere code became available with Gräfener et al. (2002).

The improved models provide a much better fit to the observed spectra, and hence lead to more reliable determination

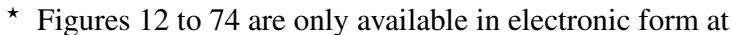
http://www. edpsciences.org of the stellar parameters. For many WN stars, a substantial revision of previous results can be expected. However, only very few WN stars have been re-analyzed so far by means of lineblanketed models (Herald et al. 2001; Marchenko et al. 2004). In the present paper we now re-analyze all Galactic WN stars for which observed spectra are available to us.

The best way to analyze a larger sample of stars systematically is first to establish "model grids", i.e. sets of model atmospheres and synthetic spectra that cover the relevant range of parameters. For WN stars we have already prepared such grids of iron-line blanketed models (Hamann \& Gräfener 2004), which will be used in the present paper.

The main objective of analyzing the Galactic WN stars is to understand the evolution of massive stars. The empirical results from our previous comprehensive analyses were in conflict with the evolutionary calculations. However, with the application of the more advanced models, we will revise the empirical stellar parameters. Moreover, the evolutionary models have also been significantly improved recently, since the effects of stellar rotation are now taken into account (Meynet \& Maeder 2003). Hence, the question of whether stellar evolution theory still conflicts with the empirical stellar parameters should be reassessed. For this purpose, we use these new evolutionary tracks for generating synthetic stellar populations and compare them with the results from our analyses of the Galactic WN sample. 
The paper is organized as follows. In Sect. 2 we briefly characterize the applied model atmospheres. The program stars and observational data are introduced in Sect. 3. The spectral analyses and their results are given in Sect. 4, together with a long list of detailed comments on individual WN stars. Our empirical results are compared with evolutionary models in Sect. 5, especially by means of a population synthesis.

\section{The models}

The Potsdam Wolf-Rayet (PoWR) model atmospheres (see Hamann \& Gräfener 2004, and references therein, for more details) are based on the "standard" assumptions (spherically symmetric, stationary mass-loss). The velocity field is pre-specified in the standard way. For the supersonic part we adopt the usual $\beta$-law, with the terminal velocity $v_{\infty}$ being a free parameter. The exponent $\beta$ is set to unity throughout this work. In the subsonic region, the velocity field is defined such that a hydrostatic density stratification is approached.

The "stellar radius" $R_{*}$, which is the inner boundary of our model atmosphere, corresponds by definition to a Rosseland optical depth of 20 . The "stellar temperature" $T_{*}$ is defined by the luminosity $L$ and the stellar radius $R_{*}$ via the Stefan-Boltzmann law; i.e. $T_{*}$ denotes the effective temperature referring to the radius $R_{*}$.

Wind inhomogeneities ("clumping") are now accounted for in a first-order approximation, assuming that optically thin clumps fill a volume fraction $f_{\mathrm{V}}$ while the interclump space is void. Thus the matter density in the clumps is higher by a factor $D=f_{\mathrm{V}}^{-1}$, compared to an un-clumped model with the same parameters. $D=4$ is assumed throughout this paper. The Doppler velocity $v_{\mathrm{D}}$, which reflects random motions on small scales ("microturbulence"), is set to $100 \mathrm{~km} \mathrm{~s}^{-1}$.

The major improvement of the models compared to those applied in Paper I is the inclusion of line blanketing by iron and other iron-group elements. About $10^{5}$ energy levels and $10^{7}$ line transitions between those levels are taken into account in the approximation of the "superlevel" approach.

The PoWR code was used to establish two grids of WN-type models (Hamann \& Gräfener 2004). One grid is for hydrogenfree stars, the other one contains $20 \%$ of hydrogen (per mass). Internet access to the synthetic spectra is provided to the community ${ }^{1}$.

Complex model ions of $\mathrm{H}, \mathrm{He}, \mathrm{N}$ III-N V and C III-C IV are taken into account in our models. The total number of NonLTE levels is 261, including 72 iron superlevels. Due to the neglect of N II, the corresponding lines that might be detectable in the coolest WNL stars are not included in our synthetic spectra. Because of the unsettled questions about a proper treatment of dielectronic recombination, this process is not taken into account.

The analyses described in the present paper mainly rely on the PoWR grid models. In addition, individual models were calculated for some stars with special settings for their hydrogen abundance and terminal wind velocity.

\section{Program stars, observational data}

The VIIth Catalogue of Galactic Wolf-Rayet Stars (van der Hucht 2001, hereafter "the WR Catalog") lists

\footnotetext{
${ }^{1}$ http://www.astro.physik. uni-potsdam.de/PoWR.html
}

227 stars in total. Among them, 127 stars $^{2}$ belong to the WN sequence considered here, while the others belong to the WC sequence or are of intermediate spectral type (WN/WC).

Many of the WN stars in the catalog are highly reddened and therefore invisible or at least very faint in visual light. Our study started with 74 WN stars for which we had sufficient spectral observations at our disposal. As we restrict the present analyses to single-star spectra, well-established binaries with composed spectra (such as WR 139 alias V444 Cyg) have been omitted from the beginning.

But so far our sample still comprised many stars that have been suspected of binarity, on more or less sound basis. We considered all these cases in detail, checking the literature and inspecting our spectral fits for indications of binarity (cf. Hamann \& Gräfener 2006). For 11 objects we finally conclude that they are indeed binaries, where the companion of the WN stars contributes more than $15 \%$ of the total light in the visual. Those stars were excluded from the present single-star analyses. They are marked in Table 2 as "composite spectrum", the reasoning given below in the "Comments on individual stars" (Sect. 4.3).

Among the remaining 63 stars (see Table 2), which we are going to analyze in the present paper, there are still four stars that are most likely close binaries, but the light contamination from the non-WN companion can be neglected. These stars are marked by the superscript ${ }^{b}$ at their WR number in Table 2, Col. (1), indicating their possible origin from closebinary evolution. Note that six stars of our present sample (WR 28, WR 63, WR 71, WR 85, WR 94, WR 107) have not been analyzed before.

The observational material is mostly the same as used in our previous papers and as was published in our atlas of $\mathrm{WN}$ spectra (see Hamann et al. 1995 for more details). These spectra were taken at the "Deutsch-Spanisches Astronomisches Zentrum (DSAZ)", Calar Alto, Spain, and at the European Southern Observatory (ESO), La Silla, Chile. For four stars (WR 6, WR 22, WR 24, WR 78) we can employ high-resolution optical spectra from the ESO archive obtained with UVES. All optical spectra are not flux-calibrated and therefore were normalized to the continuum "by eye" before being compared with normalized synthetic spectra.

Additionally, we retrieved IUE low-resolution spectra from the archive for all program stars when available. As these spectra are flux-calibrated, we can fully exploit this information. We divide the flux-calibrated observation by the (reddened) model continuum and thus obtain the comparison plot with the normalized line spectrum of the model (cf. Fig. 1, upper panel).

For fitting the spectral energy distribution, we use the IUE flux and narrow-band visual photometry ( $v, b$ bands). In addition we employ the homogeneous set of infrared photometry $(J, H$, $K$ bands) from the 2MASS survey that is available now. This extension of the wavelength range turns out to be very useful for a precise determination of the interstellar absorption, especially for highly reddened stars without UV observation.

The spectral types given in Table 2, Col. (2), are taken basically from the WR catalog. For WNE stars we append "-w" or "-s" to the classification, indicating whether the emission lines are weak or strong, respectively. The criterion is whether the

\footnotetext{
2 Actually one star less, as we believe that WR 109 (classified in the WR catalog as WN5h+?) is erroneously counted here as a WN star. The typical brightness of a WN star would imply an implausible large distance $(D M=17.4 \mathrm{mag})$, placing it beyond our Galaxy. From the binary period and the optical flickering, it has been concluded already (Steiner et al. 1988, 1999; Steiner \& Diaz 1998) that WR 109 is a cataclysmic variable $(\mathrm{CV})$.
} 

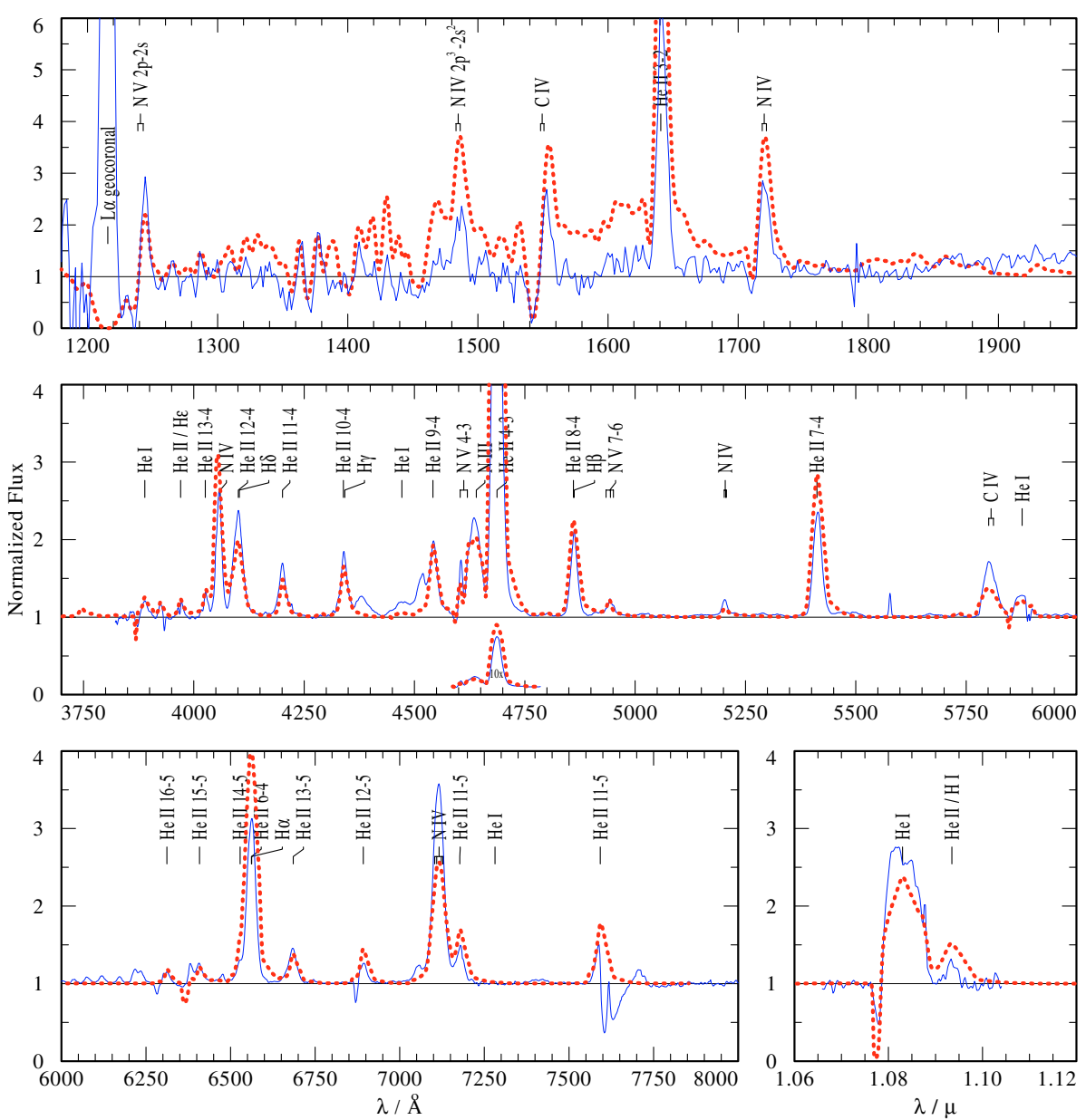

Fig. 1. Spectrum of WR 61 (solid line), together with the WNE grid model 09-14 $\left(T_{*}=63 \mathrm{kK}, \log \left(R_{\mathrm{t}} / R_{\odot}\right)=0.7\right.$, dotted line). The IUE spectrum (top panel) has been divided by the reddened model continuum (see Fig. 3), while the optical and the near-IR spectra were normalized by eye.

equivalent width of the He II line at $5412 \rightarrow \mathrm{A}$ is smaller or larger than $37 \rightarrow \mathrm{A}$ as introduced in earlier papers.

In the older classification schemes, there was a clear dichotomy between early and late WN subtypes (WNE and WNL, respectively). The early subtypes (subtype numbers up to WN6) generally showed no hydrogen, while the late subtypes (WN7 and higher) comprised the stars with rather high hydrogen abundance. With the revised classification scheme used in the WR catalog, this dichotomy is unfortunately mixed up. Now the WN6 subtype also includes a few stars that are typical members of the WNL group (namely WR 24, WR 25, and WR 85, as evident from their high hydrogen abundance and their whole spectra appearance, cf. the atlas of WR spectra from Hamann et al. 1995). Vice versa, a few stars classified as WN7 or WN8 actually belong to the WNE subclass (namely WR 55, WR 63, WR 74, WR 84, WR 91, WR 100, WR 120, and WR 123). We indicate these crosswise memberships to the WNL or WNE class in parentheses behind the spectral subtype in Col. (2) of Table 2. When discussing mean parameters of the subclasses, we assign these stars accordingly.

\section{The analyses}

\subsection{The line spectrum}

As has been described in many of our previous papers, the spectroscopic parameters of a Wolf-Rayet type stellar atmosphere are the stellar temperature $T_{*}$ and a second parameter that is related to the mass-loss rate and that we define in the form of the "transformed radius"

$R_{\mathrm{t}}=R_{*}\left[\frac{v_{\infty}}{2500 \mathrm{~km} \mathrm{~s}^{-1}} / \frac{\dot{M} \sqrt{D}}{10^{-4} M_{\odot} \mathrm{yr}^{-1}}\right]^{2 / 3}$.

For a given fixed chemical composition and a given stellar temperature $T_{*}$, synthetic spectra from Wolf-Rayet model atmospheres of different mass-loss rates, stellar radii, and terminal wind velocities yield almost the same emission-line equivalent widths, if they agree in their "transformed radius" $R_{\mathrm{t}}$. Note that the "spectroscopic parameters" $T_{*}$ and $R_{\mathrm{t}}$ do not involve the stellar distance.

Therefore the model grids (Hamann \& Gräfener 2004) are calculated with stepwise varied $\log T_{*}$ and $\log R_{\mathrm{t}}$, while all other parameters are kept fixed. Two grids have been established, one for WNE stars (without hydrogen, $v_{\infty}=1600 \mathrm{~km} \mathrm{~s}^{-1}$ ) and one for WNL stars $\left(20 \%\right.$ hydrogen, $\left.v_{\infty}=1000 \mathrm{~km} \mathrm{~s}^{-1}\right)$. For the analyses, we now plot the (normalized) observed spectrum for all stars, together with a grid model, selecting the best-fitting one. For a couple of stars we calculate individual models with suitably adapted parameters (hydrogen abundance, terminal wind speed). A typical line fit is shown in Fig. 1. We provide the fits for our whole sample as online material.

The parameters $T_{*}$ and $R_{\mathrm{t}}$ of the best-fitting model are tabulated in Table 2, Cols. (3) and (4) for all program stars. Plotting these parameters in Fig. 2 reveals that the cooler, late-type WN stars generally have thin winds and show atmospheric hydrogen, 


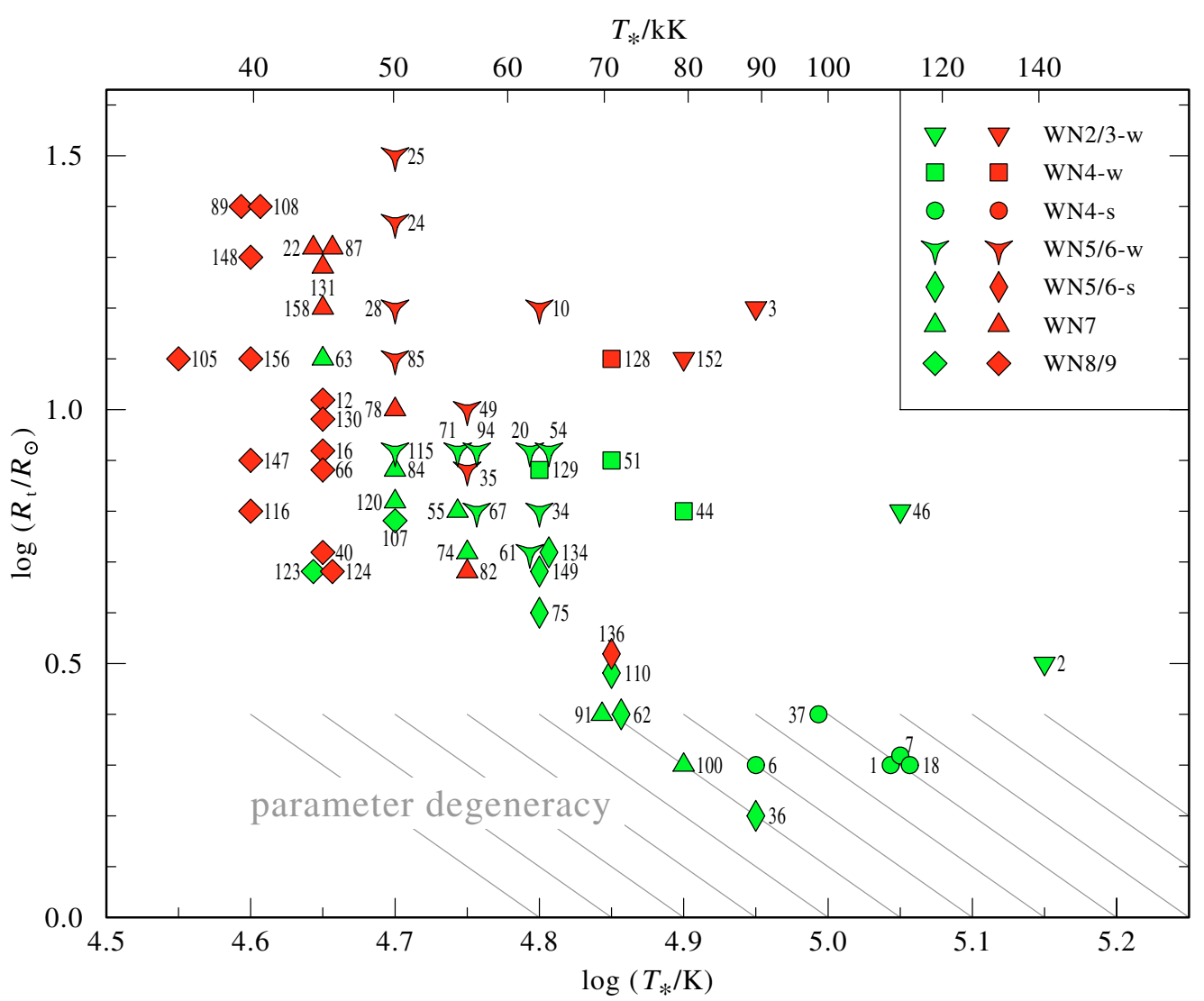

Fig. 2. Parameters of the Galactic WN stars (labels: WR-Numbers). Shown is the "transformed radius" $R_{\mathrm{t}}$ (see text) versus the stellar temperature $T_{*}$. Dark/red filled symbols refer to stars with detectable hydrogen, while light/green filled symbols denote hydrogen-free stars. The symbol shapes are coding for the spectral subtype (see inlet). In the lower, hatched part of the diagram the winds are very thick, and the parameter space is degenerate such that any stars located in this part can be arbitrarily shifted parallel to the grey lines.

while the hotter, early-type stars (WN2 ... WN6) have thicker winds and are mostly hydrogen-free.

The WNL stars populate a temperature strip between 40 and $50 \mathrm{kK}$. In comparison to Paper I, the WNL stars became about $10 \mathrm{kK}$ hotter. The WNE stars scatter over a wide range of stellar temperatures $(50 \ldots 140 \mathrm{kK})$. These temperatures have also been revised upwards in many cases compared to Paper I. The higher stellar temperatures are a consequence of the line-blanketing effects that are now taken into account in the models. The $R_{\mathrm{t}}$ values are not much different from Paper I. Note that these results are not systematically affected by the introduction of clumping, as the clumping parameter is included in the definition of the transformed radius (Eq. (1)), and models with same $R_{\mathrm{t}}$ show very similar spectra.

A few early-type WN stars with strong lines (suffix -s) fall into the domain of parameter degeneracy, where the whole emergent spectrum is formed in rapidly expanding layers. For stars with $\log \left(R_{\mathrm{t}} / R_{\odot}\right)<0.4$, the spectra depend mainly on the product $R_{\mathrm{t}} T_{*}^{2}$, but hardly on the individual values of these two parameters. Hence the location of those stars in the $R_{\mathrm{t}}-T_{*}$-plane can be shifted arbitrarily along the grey lines in Fig. 2 without spoiling the line fit. Note that along these lines the mass-loss rate is constant (in a model grid with fixed luminosity). The reason for this degeneracy is that spherically expanding atmospheres with $v(r)=$ const. and the same ratio $L / \dot{M}^{4 / 3}$ are homologues.

The terminal wind velocities (Table 2, Col. (5)) are taken from Paper I in most cases. For a number of stars we revised $v_{\infty}$ after closer inspection of our line fits. For those stars that were analyzed in detail in the meantime, we adopt $v_{\infty}$ from these sources:

WR 108: Crowther et al. (1995a);

WR 22, WR 24, WR 25, WR 78,

WR 120, WR 123, WR 124, WR 156: Crowther et al. (1995b);

WR 128, WR 152: Crowther et al. (1995d);

WR 16, WR 40: Herald et al. (2001).

The hydrogen abundance, given in Table 2 Col. (6) in terms of mass fractions $X_{\mathrm{H}}$, is also taken from Paper I or from the detailed analyses quoted in the previous paragraph. For three stars that are new in the sample, $X_{\mathrm{H}}$ is estimated for the first time (WR 28, WR 63, WR 85). If hydrogen abundances have been revised with respect to Paper I, this is mentioned in each individual case in Sect. 4.3.

\subsection{The spectral energy distribution}

Now we exploit the information provided by the spectral energy distribution (SED). We fit the whole SED from the UV (if IUE observations are available) to infrared wavelengths. The IR photometry $(J, H, K)$ can be found for all program stars in the 2MASS catalog. This information turns out to be extremely useful, especially for highly reddened objects. Visual photometry is taken from the WR catalog. Note that the monochromatic magnitudes $b, v$ as defined by Smith (1968), are used for WR photometry. The color excess in this system is related to the $E_{B-V}$ in the Johnson system by $E_{B-V}=1.21 E_{b-v}$. 


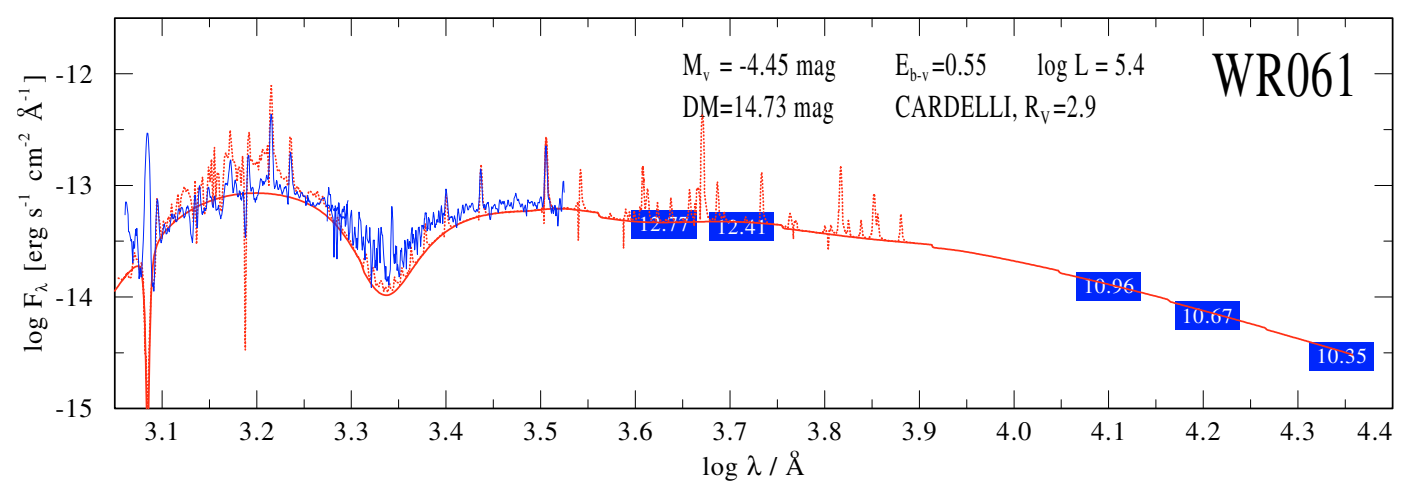

Fig. 3. Spectral energy distribution (SED). The IUE observation (noisy line) and photometry in $b, v, J, H$ and $K$ (blocks labelled with magnitude) is compared with the reddened flux (smooth line: model continuum; dotted line: model spectrum with lines) from the WNE grid model 09-14 $\left(T_{*}=63 \mathrm{kK}, \log \left(R_{\mathrm{t}} / R_{\odot}\right)=0.7\right)$. Reddening parameters and $\log L$ are suitably adjusted. The adopted absolute magnitude of $M_{v}=-4.45$ mag for the WN5 subtype implies a distance modulus of $D M=14.73 \mathrm{mag}$.

The flux from the model (as selected by the line fit described in the previous subsection) is plotted together with the observation. An example is shown in Fig. 3, while corresponding plots for the whole sample of stars are included in the online material. In order to achieve a fit, $E_{b-v}$ is suitably adjusted by hand (Table 2, Col. (7)). By default, the reddening law from Seaton (1979) is applied. It is augmented by interstellar $\mathrm{L} \alpha$ absorption in the UV and by the data from Moneti et al. (2001) in the IR. In cases where Seaton's law does not allow us to reproduce the observed SED satisfactorily, we try to improve the fit with the help of anomalous reddening. The reddening laws from Cardelli et al. (1989) and from Fitzpartick (1999) provide the adjustable parameter $R_{V}=A_{V} / E_{B-V}$, while in Seaton's law $R_{V}=3.1$ is fixed. Our optimum choice for the reddening law and $R_{V}$ (if applicable) is given in Table 2, Col. (8). Similar anomalous reddening often concerns a whole group of neighboring stars, e.g. the Car OB1 members WR 22, WR 24, and WR 25.

The chosen reddening implies extinction at the $v$ band $\left(A_{v}=\right.$ $E_{b-v}\left(1.21 R_{V}+0.36\right)$, cf. Lundstroem \& Stenholm 1984). Hence, when the distance modulus $D M$ of the object is known (see below for the discussion of distances), the absolute visual magnitude $M_{v}$ follows from the observed (apparent) magnitude $v$ via $M_{v}=v-D M-A_{v}$. Vice versa, if $D M$ is not known, it follows from an adopted absolute visual magnitude $M_{v}$.

The model flux is diluted according to the distance modulus $D M$ and reddened according to the adopted reddening law and parameter(s). At the first attempt, the model flux will not match the observed flux level. Thanks to the (approximate) scalability of the models (while the transformed radius is kept constant), the luminosity can be suitably adjusted (remember that all grid models are calculated for $\log L / L_{\odot}=5.3$ ).

The obtained stellar luminosity, radius, and mass-loss rate thus depend on the adopted stellar distance. Fortunately, about one third of the program stars are members of open clusters or associations. Those stars are identified in Table 2 by an arrow that points from Col. (9) to the right. For a few stars their unclear membership is discussed individually in Sect. 4.3.

The distance moduli for the cluster/association members (Table 2, Col. (9)) are taken from the WR catalog. Compared to Paper I, some of these distances have been revised significantly (as also discussed in Sect. 4.3 for the individual stars). The new values are not always more plausible than the older ones, but their discussion is beyond the scope of this paper. The resulting stellar parameters can be easily scaled when different distances are assumed.

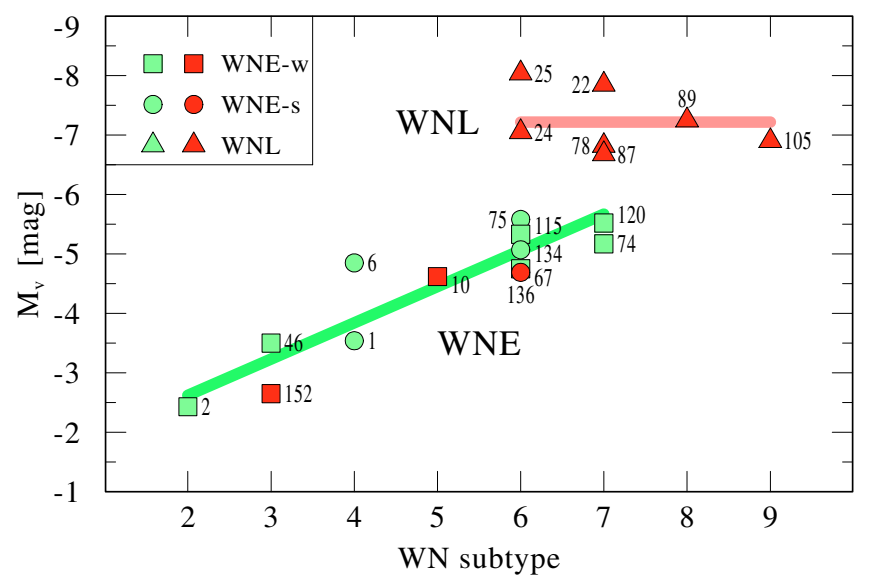

Fig. 4. Absolute visual magnitudes of Galactic WN stars with known distance. The dark (red) symbols refer to stars with detectable hydrogen, while symbols with light (green) filling stand for hydrogen-free stars. The thick lines indicate the relations which we adopted for stars of unknown distance.

Table 1. Absolute magnitudes of the different WN subtypes, as adopted for stars of unknown distance.

\begin{tabular}{lc}
\hline \hline Subtype & $M_{\mathrm{v}}[\mathrm{mag}]$ \\
\hline hydrogen-free $(W N E)$ & \\
WN2 & -2.62 \\
WN3 & -3.23 \\
WN4 & -3.84 \\
WN5 & -4.45 \\
WN6 & -5.06 \\
WN7 & -5.67 \\
with hydrogen $(W N L)$ & \\
WN6-9 & -7.22 \\
\hline
\end{tabular}

The absolute visual magnitude $M_{v}$ shows a nice correlation with the WN subtype (Fig. 4). However, it is important to distinguish between hydrogen-rich and hydrogen-poor stars because the WNE and WNL subclasses overlap in the WN6 and WN7 subtype according to the current classification scheme, as discussed in Sect. 3. All WNL stars seem to have a similar brightness, while for the WNE there is roughly a linear relation between $M_{v}$ and subtype number. On this basis we adopt a "typical" absolute magnitude for each subtype, as given in Table 1, and apply this calibration for those stars for which the distance is not known. For these stars, the arrow between Cols. (9) and (10) 
Table 2. Parameters of the Galactic single WN stars.

\begin{tabular}{|c|c|c|c|c|c|c|c|c|c|c|c|c|c|c|}
\hline WR & Spectral subtype & $\begin{array}{r}T_{*} \\
{[\mathrm{kK}]} \\
(3) \\
\end{array}$ & $\begin{array}{r}\log R_{\mathrm{t}} \\
{\left[R_{\odot}\right]} \\
(4) \\
\end{array}$ & $\begin{array}{c}v_{\infty} \\
{\left[\mathrm{km} \mathrm{s}^{-1}\right]} \\
(5)\end{array}$ & $\begin{array}{r}X_{\mathrm{H}} \\
{[\%]} \\
(6) \\
\end{array}$ & $\begin{array}{r}E_{b-v} \\
{[\mathrm{mag}]} \\
(7) \\
\end{array}$ & $\begin{array}{l}\mathrm{Law}^{a} \\
R_{V} \\
(8) \\
\end{array}$ & $\begin{array}{c}D M \\
{[\mathrm{mag}]} \\
(9) \\
\end{array}$ & $\begin{array}{r}M_{v} \\
{[\mathrm{mag}]} \\
(10)\end{array}$ & $\begin{array}{c}R_{*} \\
{\left[R_{\odot}\right]} \\
(11) \\
\end{array}$ & $\begin{array}{r}\log \dot{M}^{\dagger} \\
{\left[M_{\odot} / \mathrm{yr}\right]} \\
(12) \\
\end{array}$ & $\begin{array}{l}\log L \\
{\left[L_{\odot}\right]} \\
(13) \\
\end{array}$ & $\begin{array}{l}\frac{\dot{M} v_{\infty}}{L / c} \\
(14) \\
\end{array}$ & $\begin{array}{r}M \\
{\left[M_{\odot}\right]} \\
(15) \\
\end{array}$ \\
\hline 1 & WN4-s & 112.2 & 0.3 & 1900 & 0 & 0.67 & $\mathrm{~S}$ & 11.3 & $\rightarrow-3.54$ & 1.33 & -4.7 & 5.4 & 7.7 & 15 \\
\hline 2 & WN2-w & 141.3 & 0.5 & 1800 & 0 & 0.44 & C 3.0 & 12.0 & $\rightarrow-2.43$ & 0.89 & -5.3 & 5.45 & 1.7 & 16 \\
\hline 3 & WN3h-w & 89.1 & 1.2 & 2700 & 20 & 0.35 & C 3.4 & 12.4 & $\leftarrow-3.23$ & 2.65 & -5.4 & 5.6 & 1.2 & 19 \\
\hline 6 & WN4-s & 89.1 & 0.3 & 1700 & 0 & 0.12 & $\mathrm{~S}$ & 11.3 & $\rightarrow-4.85$ & 2.65 & -4.3 & 5.6 & 11.0 & 19 \\
\hline 7 & WN4-s & 112.2 & 0.3 & 1600 & 0 & 0.53 & $\mathrm{~S}$ & 13.4 & $\leftarrow-3.84$ & 1.41 & -4.7 & 5.45 & 5.3 & 16 \\
\hline 10 & WN5ha-w & 63.1 & 1.2 & 1100 & 25 & 0.58 & C 3.1 & 13.3 & $\rightarrow-4.62$ & 5.61 & -5.3 & 5.65 & 0.6 & 20 \\
\hline $12^{b}$ & $\mathrm{WN} 8 \mathrm{~h}+?$ & 44.7 & 1.0 & 1200 & 27 & 0.80 & C 3.7 & 14.4 & $\leftarrow-7.22$ & 21.1 & -4.1 & 6.2 & 2.7 & 44 \\
\hline 16 & WN8h & 44.7 & 0.9 & 650 & 25 & 0.55 & C 3.4 & 13.2 & $\leftarrow-7.22$ & 19.9 & -4.3 & 6.15 & 1.2 & 41 \\
\hline 18 & WN4-s & 112.2 & 0.3 & 1800 & 0 & 0.75 & C 3.6 & 11.5 & $\leftarrow-3.84$ & 1.49 & -4.6 & 5.5 & 6.5 & 17 \\
\hline 20 & WN5-w & 63.1 & 0.9 & 1200 & 0 & 1.28 & $\mathrm{~S}$ & 13.8 & $\leftarrow-4.45$ & 5.29 & -4.7 & 5.6 & 5.4 & 19 \\
\hline 21 & WN5 + O4-6 & \multicolumn{13}{|c|}{ - composite spectrum - } \\
\hline $22^{b}$ & WN7h + O9III-V & 44.7 & 1.3 & 1785 & 44 & 0.35 & C 3.8 & 12.55 & $\rightarrow-7.85$ & 31.5 & -4.2 & 6.55 & 1.8 & 74 \\
\hline 24 & WN6ha-w (WNL) & 50.1 & 1.35 & 2160 & 44 & 0.24 & C 3.1 & 12.55 & $\rightarrow-7.05$ & 19.9 & -4.4 & 6.35 & 1.7 & 54 \\
\hline $25^{b}$ & WN6h-w + ? (WNL) & 50.1 & 1.5 & 2480 & 53 & 0.63 & C 4.5 & 12.55 & $\rightarrow-8.04$ & 33.4 & -4.3 & 6.8 & 1.0 & 110 \\
\hline 28 & WN6(h)-w & 50.1 & 1.2 & 1200 & 20 & 1.20 & S & 13.1 & $\leftarrow-5.06$ & 8.89 & -5.0 & 5.65 & 1.3 & 20 \\
\hline 31 & WN4 + O8V & \multicolumn{13}{|c|}{ - composite spectrum - } \\
\hline 34 & WN5-w & 63.1 & $0.8^{1}$ & 1400 & 0 & 1.18 & $\mathrm{~S}$ & 14.1 & $\leftarrow-4.45$ & 4.72 & -4.7 & 5.5 & 4.0 & 17 \\
\hline 35 & WN6h-w & 56.2 & 0.9 & 1100 & 22 & 1.15 & $\mathrm{~S}$ & 14.2 & $\leftarrow-5.06$ & 6.67 & -4.8 & 5.6 & 2.3 & 19 \\
\hline 36 & WN5-s & 89.1 & 0.2 & 1900 & 0 & 1.00 & $\mathrm{~S}$ & 13.9 & $\leftarrow-4.45$ & 1.88 & -4.3 & 5.3 & 23.0 & 13 \\
\hline 37 & WN4-s & 100.0 & 0.4 & 2150 & 0 & 1.63 & S & 13.2 & $\leftarrow-3.84$ & 1.88 & -4.6 & 5.5 & 9.3 & 17 \\
\hline 40 & WN8h & 44.7 & 0.7 & 650 & 23 & 0.40 & C 3.4 & 13.3 & $\leftarrow-7.22$ & 17.7 & -4.1 & 6.05 & 2.5 & 35 \\
\hline 44 & WN4-w & 79.4 & 0.8 & 1400 & 0 & 0.62 & C 3.6 & 13.9 & $\leftarrow-3.84$ & 3.15 & -5.0 & 5.55 & 1.9 & 18 \\
\hline 46 & WN3p-w & 112.2 & 0.8 & 2300 & 0 & 0.30 & F 3.6 & 13.05 & $\rightarrow-3.50$ & 2.11 & -5.1 & 5.8 & 1.6 & 25 \\
\hline 47 & $\mathrm{WN} 6+\mathrm{O} 5 \mathrm{~V}$ & \multicolumn{13}{|c|}{ - composite spectrum - } \\
\hline 49 & WN5(h)-w & 56.2 & 1.0 & 1450 & 25 & 0.80 & $\mathrm{~S}$ & 15.0 & $\leftarrow-4.45$ & 5.29 & -5.0 & 5.4 & 3.2 & 15 \\
\hline 51 & WN4-w & 70.8 & 0.9 & 1500 & 0 & 1.40 & $\mathrm{~S}$ & 12.9 & $\leftarrow-3.84$ & 3.75 & -5.0 & 5.5 & 2.3 & 17 \\
\hline 54 & WN5-w & 63.1 & 0.9 & 1500 & 0 & 0.82 & $\mathrm{~S}$ & 14.1 & $\leftarrow-4.45$ & 5.29 & -4.8 & 5.6 & 3.0 & 19 \\
\hline 55 & WN7 (WNE-w) & 56.2 & 0.8 & 1200 & 0 & 0.65 & C 3.6 & 13.5 & $\leftarrow-5.67$ & 8.39 & -4.4 & 5.8 & 3.5 & 25 \\
\hline 61 & WN5-w & 63.1 & 0.7 & 1400 & 0 & 0.55 & C 2.9 & 14.7 & $\leftarrow-4.45$ & 4.21 & -4.7 & 5.4 & 5.9 & 15 \\
\hline 62 & WN6-s & 70.8 & 0.4 & 1800 & 0 & 1.73 & $\mathrm{~S}$ & 12.2 & $\leftarrow-5.06$ & 3.54 & -4.2 & 5.45 & 19.0 & 16 \\
\hline 63 & WN7 (WNE-w) & 44.7 & 1.1 & 1700 & 0 & 1.54 & C 3.1 & 12.2 & $\leftarrow-5.67$ & 11.2 & -4.6 & 5.65 & 5.3 & 20 \\
\hline 66 & WN8(h) & 44.7 & 0.9 & 1500 & 5 & 1.00 & $\mathrm{~S}$ & 14.8 & $\leftarrow-7.22$ & 19.9 & -3.9 & 6.15 & 6.2 & 41 \\
\hline 67 & WN6-w & 56.2 & 0.8 & 1500 & 0 & 1.05 & S & 12.6 & $\rightarrow-4.75$ & 5.29 & -4.6 & 5.4 & 6.8 & 15 \\
\hline 71 & WN6-w & 56.2 & 0.9 & 1200 & - & 0.38 & F 2.5 & 14.0 & $\leftarrow-5.06$ & 7.06 & -4.7 & 5.65 & 2.7 & 20 \\
\hline 74 & WN7 (WNE-w) & 56.2 & 0.7 & 1300 & 0 & 1.50 & $\mathrm{~S}$ & 13.0 & $\rightarrow-5.17$ & 5.29 & -4.6 & 5.4 & 7.2 & 15 \\
\hline 75 & WN6-s & 63.1 & 0.6 & 2300 & 0 & 0.93 & S & 13.0 & $\rightarrow-5.58$ & 5.94 & -4.1 & 5.7 & 19.0 & 22 \\
\hline 78 & WN7h & 50.1 & 1.0 & 1385 & 11 & 0.47 & S & 11.5 & $\rightarrow-6.82$ & 16.7 & -4.2 & 6.2 & 2.6 & 44 \\
\hline 82 & WN7(h) & 56.2 & 0.7 & 1100 & 20 & 1.00 & $S$ & 15.5 & $\leftarrow-7.22$ & 14.9 & -4.0 & 6.3 & 3.1 & 51 \\
\hline 84 & WN7 (WNE-w) & 50.1 & 0.9 & 1100 & 0 & 1.45 & $\mathrm{~S}$ & 13.3 & $\leftarrow-5.67$ & 8.89 & -4.6 & 5.65 & 3.2 & 20 \\
\hline 85 & WN6h-w (WNL) & 50.1 & 1.1 & 1400 & 40 & 0.82 & C 3.5 & 14.1 & $\leftarrow-7.22$ & 21.1 & -4.2 & 6.4 & 1.7 & 59 \\
\hline 87 & WN7h & 44.7 & 1.3 & 1400 & 40 & 1.70 & S & 12.3 & $\rightarrow-6.68$ & 18.8 & -4.6 & 6.1 & 1.4 & 38 \\
\hline 89 & WN8h & 39.8 & 1.4 & 1600 & 20 & 1.58 & S & 12.3 & $\rightarrow-7.25$ & 26.5 & -4.5 & 6.2 & 1.7 & 44 \\
\hline 91 & WN7 (WNE-s) & 70.8 & 0.4 & 1700 & 0 & 2.12 & $\mathrm{~S}$ & 13.2 & $\leftarrow-5.67$ & 5.00 & -4.0 & 5.75 & 14.2 & 23 \\
\hline 94 & WN5-w & 56.2 & 0.9 & 1300 & - & 1.49 & C 3.4 & 10.1 & $\leftarrow-4.45$ & 6.67 & -4.7 & 5.6 & 3.2 & 19 \\
\hline 100 & WN7 (WNE-s) & 79.4 & 0.3 & 1600 & 0 & 1.50 & $\mathrm{~S}$ & 13.0 & $\leftarrow-5.67$ & 3.97 & -4.1 & 5.75 & 12.6 & 23 \\
\hline 105 & WN9h & 35.5 & 1.1 & 800 & 17 & 2.15 & $\mathrm{~S}$ & 11.0 & $\rightarrow-6.90$ & 21.1 & -4.5 & 5.8 & 2.2 & 25 \\
\hline 107 & WN8 & 50.1 & 0.8 & 1200 & - & 1.41 & C 3.7 & 14.6 & $\leftarrow-7.22$ & 16.7 & -4.0 & 6.2 & 3.9 & 44 \\
\hline 108 & WN9h & 39.8 & 1.4 & 1170 & 27 & 1.00 & $\mathrm{~S}$ & 13.3 & $\leftarrow-7.22$ & 25.1 & -4.6 & 6.15 & 1.0 & 41 \\
\hline 110 & WN5-s & 70.8 & 0.5 & 2300 & 0 & 0.90 & C 3.5 & 10.6 & $\leftarrow-4.45$ & 3.15 & -4.3 & 5.35 & 23.2 & 14 \\
\hline 115 & WN6-w & 50.1 & 0.9 & 1280 & 0 & 1.50 & $\mathrm{~S}$ & 11.5 & $\rightarrow-5.33$ & 8.89 & -4.5 & 5.65 & 4.3 & 20 \\
\hline
\end{tabular}

in Table 2 points to the left, i.e. from $M_{v}$ to $D M$ corresponding to the flow of information.

Of course, the "typical" brightness for the WNL stars must be taken with care. The three stars in the Car OB1 association WR 22, WR 24, and WR 25 might be especially bright; at least, their hydrogen abundance is outstandingly large. Without these three stars, the mean $M_{v}$ of the remaining four WNL stars would be $-6.91 \mathrm{mag}$, i.e. $0.31 \mathrm{mag}$ fainter than adopted here (Table 1).
In Fig. 5 we plot the position of our program stars in the Galactic plane, taking the distance and the Galactic longitude (and neglecting the Galactic latitude, which is small). The location of the WN stars along the nearby spiral arms looks plausible; at least there is no obvious outlier to indicate that its distance modulus is drastically wrong.

Now with the distance modulus fixed for all program stars, we can establish the SED fits and determine the appropriate 
Table 2. continued.

\begin{tabular}{|c|c|c|c|c|c|c|c|c|c|c|c|c|c|c|}
\hline WR & Spectral subtype & $\begin{array}{r}T_{*} \\
{[\mathrm{kK}]} \\
(3)\end{array}$ & $\begin{array}{r}\log R_{\mathrm{t}} \\
{\left[R_{\odot}\right]} \\
(4)\end{array}$ & $\begin{array}{c}v_{\infty} \\
{\left[\mathrm{km} \mathrm{s}^{-1}\right]} \\
(5)\end{array}$ & $\begin{array}{r}X_{\mathrm{H}} \\
{[\%]} \\
(6) \\
\end{array}$ & $\begin{array}{r}E_{b-v} \\
{[\mathrm{mag}]} \\
(7)\end{array}$ & $\begin{array}{l}\mathrm{Law}^{a} \\
R_{V} \\
(8)\end{array}$ & $\begin{array}{c}D M \\
{[\mathrm{mag}]} \\
(9)\end{array}$ & $\begin{array}{r}M_{v} \\
{[\mathrm{mag}]} \\
(10)\end{array}$ & $\begin{array}{c}R_{*} \\
{\left[R_{\odot}\right]} \\
(11) \\
\end{array}$ & $\begin{array}{r}\log \dot{M}^{\dagger} \\
{\left[M_{\odot} / \mathrm{yr}\right]} \\
(12)\end{array}$ & $\begin{array}{l}\log L \\
{\left[L_{\odot}\right]} \\
(13)\end{array}$ & $\begin{array}{l}\frac{\dot{M} v_{\infty}}{L / c} \\
(14)\end{array}$ & $\begin{array}{r}M \\
{\left[M_{\odot}\right]} \\
(15)\end{array}$ \\
\hline 116 & WN8h & 39.8 & 0.8 & 800 & 10 & 1.75 & $\mathrm{~S}$ & 13.4 & $\leftarrow-7.22$ & 21.1 & -4.0 & 6.0 & 3.9 & 33 \\
\hline 120 & WN7 (WNE-w) & 50.1 & 0.8 & 1225 & 0 & 1.25 & S & 12.7 & $\rightarrow-5.52$ & 8.39 & -4.4 & 5.6 & 5.7 & 19 \\
\hline 123 & WN8 (WNE-w) & 44.7 & 0.7 & 970 & 0 & 0.75 & C 2.8 & 15.7 & $\leftarrow-7.22$ & 17.7 & -4.0 & 6.05 & 5.5 & 35 \\
\hline 124 & WN8h & 44.7 & 0.7 & 710 & 13 & 1.08 & C 2.9 & 14.6 & $\leftarrow-7.22$ & 16.7 & -4.1 & 6.0 & 3.0 & 33 \\
\hline 127 & $\mathrm{WN} 3+09.5 \mathrm{~V}$ & \multicolumn{13}{|c|}{ - composite spectrum - } \\
\hline 128 & WN4(h)-w & 70.8 & 1.1 & 2050 & 16 & 0.32 & C 3.6 & 12.9 & $\leftarrow-3.84$ & 3.54 & -5.2 & 5.45 & 2.2 & 16 \\
\hline 129 & WN4-w & 63.1 & 0.9 & 1320 & 0 & 0.85 & $\mathrm{~S}$ & 13.6 & $\leftarrow-3.84$ & 3.75 & -5.1 & 5.3 & 2.8 & 13 \\
\hline 130 & WN8(h) & 44.7 & 1.0 & 1000 & 12 & 1.46 & S & 13.8 & $\leftarrow-7.22$ & 22.1 & -4.2 & 6.25 & 1.8 & 47 \\
\hline 131 & WN7h & 44.7 & 1.3 & 1400 & 20 & 1.15 & S & 14.9 & $\leftarrow-7.22$ & 23.7 & -4.4 & 6.3 & 1.3 & 51 \\
\hline 133 & WN5 + O9I & \multicolumn{13}{|c|}{ - composite spectrum - } \\
\hline 134 & WN6-s & 63.1 & 0.7 & 1700 & 0 & 0.47 & C 3.4 & 11.2 & $\rightarrow-5.07$ & 5.29 & -4.4 & 5.6 & 7.8 & 19 \\
\hline 136 & WN6(h)-s & 70.8 & 0.5 & 1600 & 12 & 0.45 & $\mathrm{~S}$ & 10.5 & $\rightarrow-4.69$ & 3.34 & -4.5 & 5.4 & 10.9 & 15 \\
\hline 138 & WN5-w + B? & \multicolumn{13}{|c|}{ - composite spectrum - } \\
\hline 139 & WN5 + O6II-V & \multicolumn{13}{|c|}{ - composite spectrum - } \\
\hline 141 & WN5-w + O5V-III & \multicolumn{13}{|c|}{ - composite spectrum - } \\
\hline 147 & WN8(h) + B0.5V & 39.8 & 0.9 & 1000 & 5 & 2.85 & $\mathrm{~S}$ & 10.4 & $\leftarrow-7.22$ & 29.8 & -3.8 & 6.3 & 3.6 & 51 \\
\hline $148^{b}$ & $\mathrm{WN} 8 \mathrm{~h}+\mathrm{B} 3 \mathrm{IV} / \mathrm{BH}$ & 39.8 & 1.3 & 1000 & 15 & 0.83 & C 3.0 & 14.4 & $\leftarrow-7.22$ & 26.5 & -4.5 & 6.2 & 1.0 & 44 \\
\hline 149 & WN5-s & 63.1 & 0.7 & 1300 & 0 & 1.42 & $\mathrm{~S}$ & 13.3 & $\leftarrow-4.45$ & 3.97 & -4.7 & 5.35 & 5.2 & 14 \\
\hline 151 & WN4 + O5V & \multicolumn{13}{|c|}{ - composite spectrum - } \\
\hline 152 & WN3(h)-w & 79.4 & 1.1 & 2000 & 13 & 0.50 & C 3.2 & 12.2 & $\rightarrow-2.65$ & 2.23 & -5.5 & 5.25 & 1.7 & 12 \\
\hline 155 & WN6 + 09II-Ib & \multicolumn{13}{|c|}{ - composite spectrum - } \\
\hline 156 & WN8h & 39.8 & 1.1 & 660 & 27 & 1.22 & $\mathrm{~S}$ & 13.3 & $\leftarrow-7.22$ & 23.7 & -4.5 & 6.1 & 0.9 & 38 \\
\hline 157 & WN5-w (+B1II) & \multicolumn{13}{|c|}{ - composite spectrum - } \\
\hline 158 & WN7h + Be? & 44.7 & 1.2 & 900 & 30 & 1.08 & $\mathrm{~S}$ & 14.3 & $\leftarrow-7.22$ & 25.1 & -4.5 & 6.35 & 0.7 & 54 \\
\hline
\end{tabular}

${ }^{a}$ Column (8) Applied reddening law: $\mathrm{S}=$ Seaton (1979), $\mathrm{C}=$ Cardelli et al. (1989), $\mathrm{F}=$ Fitzpatrick (1999); for the last two, the given number is the adopted $R_{V}$.

${ }^{b}$ Binary system in which the non-WR component contributes more than $15 \%$ of the flux in the visual.

${ }^{\dagger}$ Mass-loss rates are for an adopted clumping factor of $D=4$.

scaling factor of the model flux. Thus we obtain the stellar luminosity $L$ and, by means of Eq. (1), the mass-loss rate $\dot{M}$ and the stellar radius $R_{*}$ as given in Cols. (11)-(13) of Table 2 .

Compared to the results of Paper I, the luminosities obtained now are significantly larger, especially for the WNL stars. This is only partly due to the $M_{v}$ calibration discussed above. More important, this is a consequence of the higher stellar temperatures that we derive now with the line-blanketed model atmospheres. The mass-loss rates became lower than in Paper I, simply because of the adopted clumping with $D=4$.

The most characteristic property of Wolf-Rayet stars is their strong mass loss. Figure 6 shows the empirical mass-loss rate obtained for our sample. The WR mass-loss rates are key ingredients in various astrophysical models, e.g. of stellar evolution or galactic evolution. In recent years, the empirical mass-loss formula from Nugis \& Lamers (2000) has become the most popular. We plot their relation in Fig. 6 for comparison. The WNE stars should be described by the upper line, while most WNL stars should lie between that relation and the lower line, according to their hydrogen abundance between zero and $40 \%$ (mass fraction). For WNE stars, the formula roughly gives the observed mass-loss rates. But the scatter is large, and there is actually not much of a correlation between $\dot{M}$ and $L$. The same holds for the WNL stars. Even worse, most observed mass-loss rates of the latter are significantly lower than described by the Nugis \& Lamers formula. We conclude that more parameters (in addition to $L$ and $X_{\mathrm{H}}$ ) are involved in controlling the mass loss from WN stars.
Note that the empirical mass-loss rates scale inversely with the square root of the adopted clumping contrast, i.e. $\dot{M} \propto D^{-1 / 2}$. Our choice of $D=4$ is rather conservative. There are indications that the clumping is actually even stronger, and hence the massloss rates might still be over-estimated generally by a factor of 2 or 3.

We also calculate the ratio between the wind momentum and the momentum of the radiation field, $\eta=\dot{M} v_{\infty} c / L$ (Col. (14)). This number cannot exceed unity for a radiation-driven wind if each photon can only be scattered (or absorbed) by one spectral line. Models accounting for multiple scattering are not bound to that limit. The first fully self-consistent hydrodynamical model for a Wolf-Rayet star has been presented only recently by Gräfener \& Hamann (2005). Their model for the WC5 prototype star WR 111 exceeds the single-scattering limit moderately $(\eta=2.54)$. WNL models presently under construction (Gräfener \& Hamann, in preparation) achieve "efficiency numbers" $\eta$ between 1 and 2, typically. Our empirical values for $\eta$ are quite different for the different subclasses; the arithmetic mean for the WNE-w and WNL subclasses is 3.4 and 2.2, respectively. This is nearly reached by the self-consistent models (the remaining difference might be attributed to incomplete opacities). However, the mean $\eta$ of the WNE-s subclass is 12.5, which is much higher than any of our radiation-driven models can explain so far.

Remember that in Paper I we arrived at much higher values for $\eta(9,9$, and 29 for the WNL, WNE-w, and WNEs subclass, respectively). The smaller "efficiency numbers" result from the combined effect of higher luminosities (due to line-blanketing) 


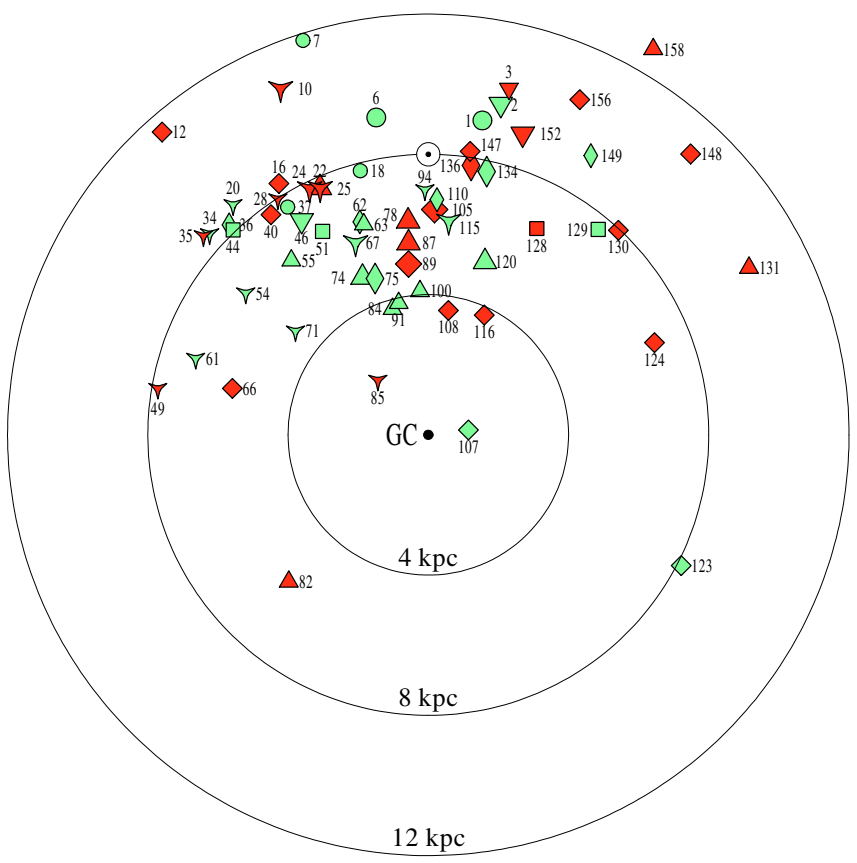

Fig. 5. Galactic position of the analyzed WN stars. The meaning of the symbols is the same as in Fig. 2. Stars with distances known from cluster/association membership are represented by bigger symbols, while smaller symbols rely on our $M_{v}$ calibration of the WN subtypes. The sun $(\odot)$ and the Galactic Center ("GC") are indicated.

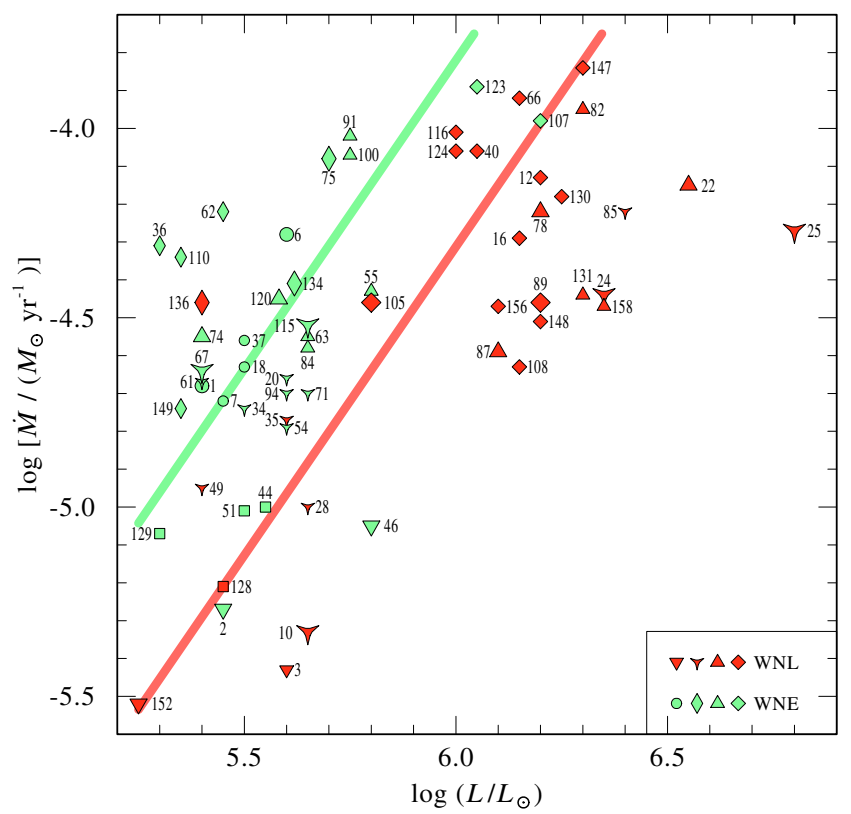

Fig. 6. Empirical mass-loss rate versus luminosity for the Galactic WN stars. Dark (red) filled symbols refer to stars with detectable hydrogen (WNL), while light (green) filled symbols denote hydrogen-free stars (WNE). The thick straight lines correspond to the mass loss - luminosity relation proposed by Nugis \& Lamers (2000) for hydrogen-free WN stars (upper line) and for a hydrogen mass fraction of $40 \%$ (lower line).

and lower mass-loss rates (due to clumping). Even higher clumping contrast could perhaps reduce $\eta$ by another factor of two.

For completeness we derive a stellar mass from the luminosity by means of the mass-luminosity relation for helium stars from Langer (1989). Note that this relation might not be adequate for stars showing hydrogen.
The discussion of the luminosities is deferred to Sect. 5 in the context of stellar evolution. First we comment on the individual stars of our program, as far as they deserve special remarks.

\subsection{Comments on individual stars}

WR 1 belongs to the Cas OB7 association, for which the distance modulus has been revised from $D M=12.1 \mathrm{mag}$, as used in Paper I, to 11.3 mag (Garmany \& Stencel 1992).

WR 2 is marked as a visual binary in the WR catalog. But according to Hipparcos, the companion is at $13.8^{\prime \prime}$ separation and should therefore not contaminate the observed spectra.

A short glance at the atlas of Galactic WN spectra (Hamann et al. 1995) reveals that this weak-lined WN2 star is unique with respect to the shape of its line profiles. Such round profiles are not reproduced by any of our models, unless very rapid rotation is assumed. Figure 7 compares observed line profiles of WR 2 with model profiles before and after flux convolution (which is only a very crude approach to a rotating wind, admittedly) with $1900 \mathrm{~km} \mathrm{~s}^{-1}$ rotational velocity. Note that WR 2 , the only Galactic star classified as WN2, is the hottest $\left(T_{*}=140 \mathrm{kK}\right)$ and most compact $\left(R_{*}=0.89 R_{\odot}\right)$ star of our sample. A rotational velocity of $1900 \mathrm{~km} \mathrm{~s}^{-1}$ would imply that the star is spinning at its break-up limit.

WR 3 is classified as WN3+O4 in the WR catalog, but there is not much evidence for its binary status. Neither Massey \& Conti (1981) nor Marchenko et al. (2004) could confirm the previously claimed periodic radial velocity variation. Our spectral fit gives no evidence of a binary contamination. Thus we consider WR 3 as a single star.

From our subtype calibration of $M_{v}$ we infer a distance of $D M=12.36 \mathrm{mag}$, which is considerably smaller than the 13.17 mag obtained by Arnal \& Roger (1997) from studying the interstellar medium towards WR 3. If their distance is right, WR 3 would be unusually bright for its spectral subtype.

In contrast to Paper I, we find that a model with about $20 \%$ hydrogen gives a slightly better fit of the $\mathrm{H} / \mathrm{He}$ blends with $\mathrm{H} \alpha$ and $\mathrm{H} \beta$ than do hydrogen-free models, supporting the corresponding result of Marchenko et al. (2004). We therefore change the spectral type to WN3h-w.

The stellar temperature that we obtain $(89 \mathrm{kK})$ is somewhat higher than the $77 \mathrm{kK}$ given by Marchenko et al. (2004). We also obtain a higher luminosity (by $0.2 \mathrm{dex}$ ), due to the higher absolute visual magnitude that we adopt for this subtype.

WR 6 shows considerable photometric, spectral, and polarimetric variability with a period of about $3.7 \mathrm{~d}$; but this period is unstable, and there are also epochs without variations. Morel et al. (1997) presented arguments for why the variability cannot be attributed to the presence of a compact companion, but is instead due to the rotation of a structured wind. Its X-ray flux is strong for a single WN star, but too low for a high-mass X-ray binary and within the usual $L_{\mathrm{X}}-L_{\mathrm{bol}}$-relation for single $\mathrm{O}$ stars (Oskinova 2005). The radio spectrum is thermal (Dougherty \& Williams 2000). Hence we consider WR 6 as a single star.

WR 10 is designated as a visual binary, with spectral type WN5ha $(+\mathrm{A} 2 \mathrm{~V})$, in the WR catalog. However, the companion A star (for its spectrum, see Niemela et al. 1999) is at 3.7" separation and $1.7 \mathrm{mag}$ fainter (Hipparcos photometry). Therefore a spectral contamination should be small even when the companion is within the aperture of the instrument. Our models can fit the SED and the line spectrum perfectly. 

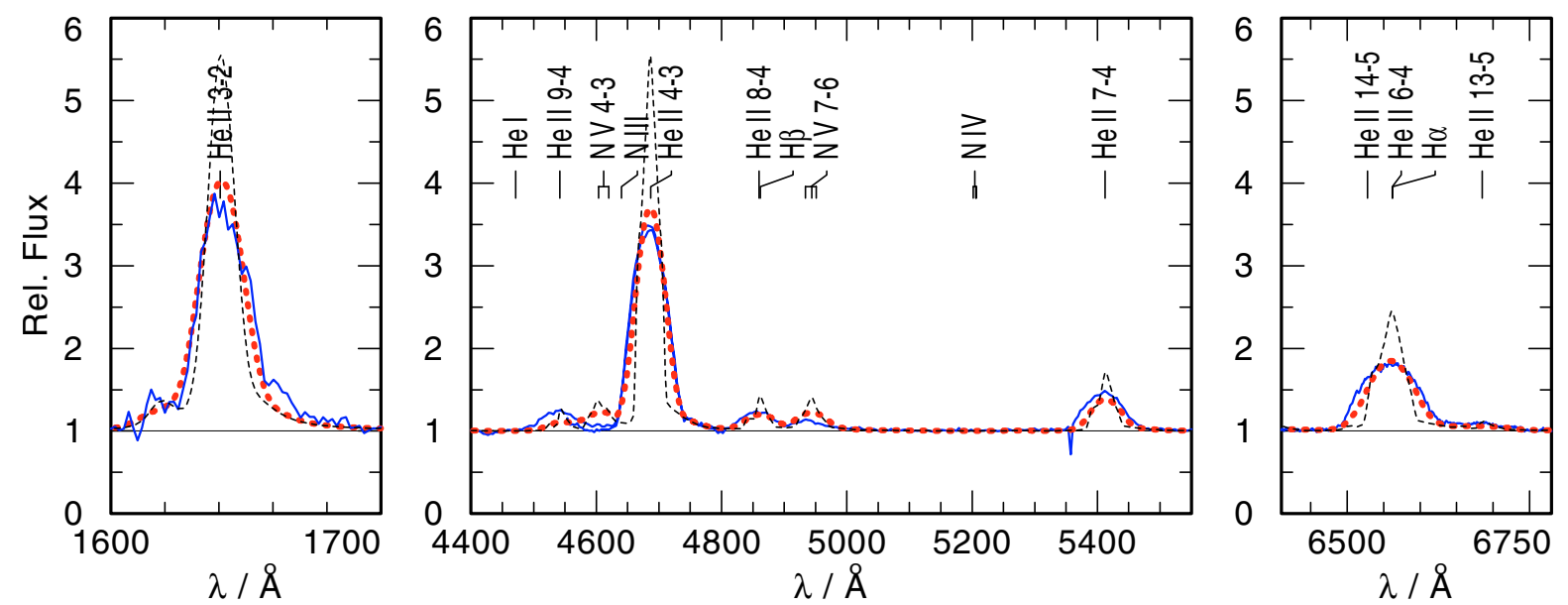

Fig. 7. Observed line profiles of WR 2 (solid line), compared to a model without rotation (dashed) and after convolution corresponding to $v_{\text {rot }} \sin i=$ $1900 \mathrm{~km} \mathrm{~s}^{-1}$ (dotted).

WR 12 is classified as WN8h+?, based on radial velocity variations (Niemela 1982; Rauw et al. 1996a) and the eclipse lightcurve (Lamontagne et al. 1996) with a period of $23.9 \mathrm{~d}$. However, no lines from the companion are seen (SB1). As our models reproduce the line spectrum and the SED (the latter with anomalous reddening), we assume that the spectrum is not contaminated, but the star might have evolved in a close binary system.

As the membership in the cluster Bo7 is only "possible" (Lundstroem \& Stenholm 1984), we instead assume the typical WN8 brightness for that star and deduce the spectroscopic distance. Our reddening estimate compares well with the average reddening of the cluster $\left(E_{b-v}=0.74 \mathrm{mag}\right)$, but the cluster distance $(D M=13.8 \mathrm{mag})$ would result in an $0.6 \mathrm{mag}$ fainter $M_{v}$ than the "typical" WN8 brightness from our subtype calibration.

WR 16 shows substantial, but not periodic photometric variability (Balona et al. 1989), and polarimetric evidence of a non-spherical outflow (Schulte-Ladbeck 1994). The thermal radio spectral index (Dougherty \& Williams 2000) and the nondetection of X-rays (Oskinova 2005) speak against binarity. WR 16 has been analyzed in detail with line-blanketed models by Herald et al. (2001). We adopt $v_{\infty}$ and $X_{\mathrm{H}}$ from that paper. Their analysis agrees well with our present results, except that they assume a different absolute visual magnitude for that spectral subtype.

WR 18 is considered by Lundstroem \& Stenholm (1984) as a "possible" member of the Car OB1 association $(D M=$ $12.55 \mathrm{mag}$ ). However, with the typical brightness from our subtype calibration, the star appears much closer $(D M=11.5 \mathrm{mag})$. Therefore we reject the membership. On the other hand, its reddening, $E_{b-v}=0.75 \mathrm{mag}$, is even higher than the typical reddening in Car OB1 (cf. the definite members WR 22, WR 24, and WR 25).

WR 21, classified as WN5 + O4-6, is a SB2 spectroscopic binary with a well-known period of $8.25 \mathrm{~d}$, showing weak O-star absorption features moving in antiphase to the WR emission lines (Niemela \& Moffat 1982). Lamontagne et al. (1996) use the eclipse lightcurve to derive that $84 \%$ of the visual brightness must be attributed to the $\mathrm{O}$ star companion. Therefore we must omit that star from our present analysis.

WR 22, classified as WN7h+O9III-V in the WR catalog, is definitely a SB2 binary with a period of $P=80.4 \mathrm{~d}$. The object has a thermal radio spectrum (Dougherty \& Williams 2000) and strong X-ray emission (Oskinova 2005). From the diluted O star absorption lines visible in the composite spectrum, Rauw et al. (1996b) estimate that the O star companion contributes about $1 / 8$ to the the flux in the visual. We ignore this contribution in our spectral analysis. The stellar temperature that we obtain is about $10 \mathrm{kK}$ higher than from previous, un-blanketed analyses (Paper I; Crowther et al. 1995b). We adopt the hydrogen abundance of $44 \%$ by mass from the latter work, while our Paper I arrived at a slightly lower value $(40 \%)$.

WR 22 is a definite member of Car OB1 (Lundstroem \& Stenholm (1984). The reddening parameters from our analysis conform closely with the anomalous reddening of that region. The distance of the Car OB 1 association has been increased considerably from $D M=12.1 \mathrm{mag}$ as used in Paper I to 12.55 mag (Massey \& Johnson 1993). With this revised distance, WR 22 becomes very luminous. Simple application of the mass-luminosity relation for helium stars yields a stellar mass of $74 M_{\odot}$, while from the binary orbit the mass of the WR component has been determined to $72 M_{\odot}$ (Rauw et al. 1996b) or $55 M_{\odot}$ (Schweickhardt et al. 1999).

WR 24 is also a Car OB 1 member, and the revised distance leads to a very high luminosity, as in the case of WR 22.

WR 25 is classified as WN6h+O4f in the WR catalog, but its binary status remains debated. The photometric and polarization variability is small and stochastic (Drissen et al. 1992). Raassen et al. (2003) found no X-ray variations over the history of X-ray astronomy. Only very recently, the detection of radial velocity variations with a period of about 200 days has been reported (Gamen \& Gosset, private communication).

The line spectrum of WR 25 can be perfectly matched by our single-star model, including the absorption features. However, the bright near-IR photometry makes it difficult to fit the SED. As already stated by Crowther et al. (1995b) this requires an anomalous reddening law with large parameter $R_{v}$. Note that this is also the case for the neighboring stars in Car OB1, WR 22 and WR 24, but less extreme (see Table 2). WR 25 is also affected by the upward revision of the distance to the Car OB1 association (see WR 22).

The stellar temperature we obtain is drastically higher $(50 \mathrm{kK})$ than found in the previous, un-blanketed analyses (Paper I: $36 \mathrm{kK}$, Crowther et al. 1995b: $31 \mathrm{kK}$ ). The consequence of the increased distance, high (anomalous) reddening and the 
revised stellar temperature is a luminosity of $\log L / L_{\odot}=6.8$, which would advance WR 25 to the most-luminous galactic WR star and to one of the most-luminous stars of our whole Galaxy. If WR 25 is indeed that luminous, its paramount X-ray brightness is just in line with the usual $L_{X}-L_{\text {bol }}$-relation for single O stars (Oskinova 2005). No conclusion can be drawn from the radio spectral index of WR 25 (Dougherty \& Williams 2000).

Alternatively, the high near-IR flux could be attributed to a cool companion. If a blackbody of $5 \mathrm{kK}$ is assumed to contribute to the near-IR, the SED can be fitted with much lower reddening, and the deduced stellar luminosity is only $\log L / L_{\odot}=5.8$ (see Hamann \& Gräfener 2006).

As revealed by observations with the Spitzer Space Telescope, the mid-IR $(\lambda>10 \mu \mathrm{m})$ spectrum of WR 25 is dominated by strong emission that cannot be attributed to the WR star (Barniske et al. 2006).

We decide to keep WR 25 in our analyzed sample, as the visual spectrum is not contaminated by a binary. However, reddening and derived luminosity are uncertain, and we leave the star out of the sample when comparing it with tracks for single-star evolution.

WR 28 has never been analyzed before. It is classified as WN6(h) + OB? in the WR catalog, but the binary suspicion seems to be based only on the weakness ("dilution") of the emission lines. As our models can reproduce the observed spectrum, we conclude that there is no evidence of binarity. For the $M_{v}$ calibration, we put WR 28 in the hydrogen-free WNE6-w subclass. Its hydrogen content is low, and adopting the high brightness of WNL stars would imply an implausible luminosity and distance for this star.

WR 31, classified as WN4 + O8V in the WR catalog, is an SB2 system (Gamen \& Niemela 1999) with a period of $4.8 \mathrm{~d}$, and it also has a visual companion that is 2.5 HIPPARCos magnitudes fainter at 0.6" separation. Lamontagne et al. (1996) use the eclipse lightcurve to derive that $72 \%$ of the visual brightness must be attributed to the $\mathrm{O}$ star companion. Therefore we must omit that star from our present analysis.

WR 35 is classified as WN6h + OB? in the WR catalog, but the suspicion of binarity seems to be based only on the weakness ("dilution") of the emission lines. As our models can reproduce the observed spectrum, we conclude that there is no evidence of binarity.

WR 36 is classified as WN5-6 + OB? in the WR catalog, but the suspicion of binarity seems to be based only on the weakness ("dilution") of the emission lines. As our models can reproduce the observed spectrum, we conclude that there is no evidence of binarity. The spectrum shows no N III lines, therefore we change the classification to WN5 in accordance with Smith et al. (1996).

WR 40 shows a large variability in all spectral bands and in polarimetry. Matthews \& Moffat (1994) conclude that the apparently chaotic variations are in fact harmonics of two periods. The variability might indicate the presence of a compact companion, but the absence of X-ray emission (Oskinova 2005) speaks against such assumption. Herald et al. (2001) have analyzed WR 40 with line-blanketed models, including the determination of various elemental abundances. We adopt $v_{\infty}$ and $X_{\mathrm{H}}$ from that paper. The stellar temperature they obtain is similar to our result. Their value for $\log L$ is lower because they assume a lower absolute magnitude for that subtype than our subtype calibration predicts.
WR 44 is classified as WN4 + OB? in the WR catalog, but the binary suspicion seems to be based only on the weakness ("dilution") of the emission lines. As our models can reproduce the observed spectrum, we conclude that there is no evidence of binarity.

WR 46 is classified as WN3p+OB? in the WR catalog. A radial velocity period of $0.31 \mathrm{~d}$ was found by Niemela et al. (1995) and later revised by Marchenko et al. (2000) to $0.329 \mathrm{~d}$. Both papers propose an evolved binary with a neutron star companion and an accretion disk. Additionally, Steiner \& Diaz (1998) found a photometric period of $7.46 \mathrm{~d}$. Veen et al. (2002) favor a nonradial pulsator instead of the binary scenario. In X-rays WR 46 is unusually bright for a WNE star, but this agrees with the usual $L_{\mathrm{X}}-L_{\mathrm{bol}}$-relation for single $\mathrm{O}$ stars (Oskinova 2005). The stellar spectrum does not appear to be composite. We consider WR 46 as a single star until the binary status is eventually confirmed. $v_{\infty}=2300 \mathrm{~km} \mathrm{~s}^{-1}$ is kept from Paper I, because our fits indicate that the $2450 \mathrm{~km} \mathrm{~s}^{-1}$ from Crowther et al. (1995d) is somewhat too high.

According to Tovmassian et al. (1996), WR 46 is a member of the Cru OB4.0 association, and therefore the $D M$ was slightly revised from $D M=13.0$ mag to $13.05 \mathrm{mag}$.

WR 47, classified as WN6 + O5V in the WR catalog, is an SB2 system (Gamen \& Niemela 1999) with a period of $6.2 \mathrm{~d}$. Lamontagne et al. (1996) derive from the eclipse lightcurve that $61 \%$ of the visual brightness must be attributed to the $\mathrm{O}$ star companion. Therefore we must omit that star from our present analysis.

WR 51 is classified as WN4 + OB? in the WR catalog, but the binary suspicion seems to be based only on the weakness ("dilution") of the emission lines. As our models can reproduce the observed spectrum, we conclude that there is no evidence of binarity.

The WR catalog lists WR 51 as a possible member of the Anon Cen OB association. However, Lundstroem \& Stenholm (1984) already noted that this star has much higher reddening. Our analysis yields $E_{b-v}=1.4$ while the average reddening in Anon Cen OB is $E_{b-v}=0.22$ (Lundstroem \& Stenholm 1984), thus confirming that WR 51 is a background object.

WR 63 has not been analyzed before. It is classified as WN7 + OB in the WR catalog, but the binary suspicion seems to be based only on the weakness ("dilution") of the emission lines and the presence of absorption features. As our models can reproduce the observed spectrum, we conclude that there is no evidence of binarity.

WR 66 is classified as WN8(h) + cc? in the WR catalog. From the observed variability, Antokhin et al. (1995) suggest the possibles existence of a compact companion spiraling in towards the WR star. In contrast, Rauw et al. (1996b) favor the non-radial pulsation aspect as proposed for many WN8. We conclude that the evidence of close binarity is insufficient. The star also has a visual companion, 1.05 HIPPARCOS magnitudes fainter and separated by $0.4^{\prime \prime}$.

Lundstroem \& Stenholm (1984) and the WR catalog list WR 66 as a possible member of the association Anon Cir OB1. While the reddening would be compatible, the distance module of Cir OB1 (12.57 mag) would lead to an atypically low brightness for a WN8 subtype. Therefore we instead consider WR 66 as a background object.

WR 67 is classified as WN6 + OB? in the WR catalog, but the binary suspicion seems to be based only on the weakness ("dilution") of the emission lines. As our models can reproduce the 
observed spectrum, we conclude that there is no evidence of binarity. WR 67 belongs to the Cir OB1 association, for which the distance has been slightly revised from $D M=12.8$ mag to $12.6 \mathrm{mag}$ (Lortet et al. 1987).

WR 71, classified as WN6 + OB? in the WR catalog, is suspected as a binary because of "diluted emission lines" and because Isserstedt et al. (1983) found a photometric and spectroscopic period of $7.69 \mathrm{~d}$. Balona et al. (1989) could confirm the photometric variability, but found large scatter from a periodicity. Marchenko et al. (1998b) could not even see any variability. We consider the evidences of binarity as not being sufficient. As we have no optical spectrum of WR 71 at our disposal, the analysis was based on the IUE data and no hydrogen abundance could be determined.

WR 78 is quoted here with a hydrogen abundance of $11 \%$ by mass from Crowther et al. (1995b), while our Paper I gives a slightly higher value $(15 \%)$.

WR 85 has not been analyzed before. It is classified as WN6h + OB? in the WR catalog, but the binary suspicion seems to be based only on the weakness ("dilution") of the emission lines. As our models can reproduce the observed spectrum, we conclude that there is no evidence of binarity. The VB (visual binary) noted in the WR catalog refers to a bright ( $V=6.5 \mathrm{mag}$ ) $\mathrm{G}$ star that is $15^{\prime \prime}$ apart and should not confuse the observed spectra.

WR 87 is classified as WN7h + OB in the WR catalog, but the binary suspicion seems to be based only on the weakness ("dilution") of the emission lines and spectral absorption features. As our models can reproduce the observed spectrum, we conclude that there is no evidence of binarity.

WR 89 is classified as WN8h + OB in the WR catalog, but the binary suspicion seems to be based only on the weakness ("dilution") of the emission lines and spectral absorption features. The radio spectral index is thermal (Dougherty \& Williams 2000). As our models can reproduce the observed spectrum, we conclude that there is no evidence of binarity. The visual companion ("VB") mentioned in the WR catalog is 10 " away and too faint to confuse the observation.

WR 94 is new in our sample. The observations available to us do not allow determination of the hydrogen abundance.

WR 105, one of the two WN9h stars in our sample, shows a non-thermal radio spectral index (Dougherty \& Williams 2000), but as there are no other indications of binarity we consider it as a single star.

WR 107 is new in our sample. The observations available to us do not allow determination of the hydrogen abundance.

WR 108 is classified as WN9h+OB in the WR catalog. However, no radial variations were found by Lamontagne et al. (1983). The binary suspicion seems to be based only on the weakness ("dilution") of the emission lines and spectral absorption features. As our spectral fit is perfectly able to reproduce the SED and the line spectrum, we conclude that there is no evidence of a luminous companion. The same conclusion was drawn by Crowther et al. (1995a), who presented a detailed analysis of that star. However, their un-blanketed analysis (as well as ours in Paper I) gave a significantly lower $T_{*}(\approx 30 \mathrm{kK})$ than the $40 \mathrm{kK}$ obtained in the present paper. Consequently, we obtain a higher luminosity. In agreement with Crowther et al. (1995a), we do not consider WR 108 as a member of Sgr OB1 $(D M=11.0 \mathrm{mag})$, but assume the $M_{v}$ from our subtype calibration. $v_{\infty}$ and $X_{\mathrm{H}}$ are taken from Crowther et al. (1995a).

WR 110 requires an anomalous reddening to fit the SED, which shows relatively high flux in the near-IR. Its X-ray flux is strong for a single WN star, but lies on the usual $L_{\mathrm{X}}-L_{\mathrm{bol}}$-relation for single O stars (Oskinova 2005). As there are no further indications of binarity, we consider the star as single. We classify this star as WN5 (WR catalog: WN5-6).

This star is listed in Lundstroem \& Stenholm (1984) as a possible member of the Sgr OB1 association ( $D M=11.0 \mathrm{mag})$. As we generally do not adopt such "possible" memberships, we consider the distance as unknown and employ the $M_{v}$ from our subtype calibration instead. However, it turns out that the obtained distance $(D M=10.6 \mathrm{mag})$ is similar to the $\mathrm{Sgr} \mathrm{OB} 1$ value. For the color excess $E_{b-v}$, we obtain $0.90 \mathrm{mag}$, while Lundstroem \& Stenholm (1984) give $0.6 \mathrm{mag}$ as average for that region of the association. Thus the question of the membership remains open.

WR 115 is classified as WN6 + OB? in the WR catalog, but the binary suspicion seems to be based only on the weakness ("dilution") of the emission lines. As our models can reproduce the observed spectrum, we conclude that there is no evidence of binarity. WR 115 belongs to the Ser OB1 association, for which the distance has been slightly revised from $D M=11.7$ mag to $11.5 \mathrm{mag}$ (Hillenbrand et al. 1993).

WR 120 is a "possible" member of the open cluster Do 33 (Lundstroem \& Stenholm 1984). Adopting the distance of that cluster yields an absolute visual magnitude for that star, which is in nice agreement with our subtype calibration. Moreover, we find a color excess $E_{b-v}$ of $1.25 \mathrm{mag}$, almost identical with the average cluster reddening (1.3 mag, Lundstroem \& Stenholm 1984). Thus WR 120 is most likely a true cluster member.

WR 123 is a WN8 star with unclear binary status. Moffat \& Shara (1986) report a photometric variability and two visual companions at $5^{\prime \prime}$ and $18^{\prime \prime}$ apart, which are too faint to cause the observed variations. Therefore they discuss the possibility of a compact companion. Alternatively, Marchenko et al. (1998b) and Marchenko \& Moffat (1998) attribute the variability to nonradial pulsations. We consider WR 123 as a single star.

WR 124 is of WN8h subtype and very similar in variability to WR 123. It also remains in our single-star sample as the evidence of binarity is weak. The hydrogen abundance from Paper I has been confirmed by Crowther et al. (1995b).

WR 127 is an SB2 binary system (WN3 + O9.5V) with a period of $9.6 \mathrm{~d}$. Lamontagne et al. (1996) use the eclipse lightcurve to derive that $58 \%$ of the visual brightness must be attributed to the $\mathrm{O}$ star companion. Therefore we must omit that star from our present analysis.

WR 128 is classified as WN4(h)+OB? in the WR catalog. Antokhin and Cherepashchuk (1985) found this star to be photometric variable with $P=3.871 \mathrm{~d}$ and eclipses in the $\mathrm{V}$ band. They concluded that a neutron star with an optical bright accretion disk accompanies the WR star. We consider WR 128 as a single star until a compact companion has been confirmed. The hydrogen abundance and $v_{\infty}$ in Table 2 are taken from the analysis by Crowther et al. (1995d).

WR 131 is classified as WN7h + OB in the WR catalog, but the binary suspicion seems to be based only on the weakness ("dilution") of the emission lines and the presence of absorption features. As our models can reproduce the observed spectrum, we conclude that there is no evidence of binarity. 
WR 133, classified as WN5 + O9I, is a SB2 binary system with a period of $112 \mathrm{~d}$. According to Underhill \& Hill (1994), the O star contributes between $11 \%$ and $70 \%$ to the total visual brightness. Because of this contamination we omit this star from the analyzed sample.

WR 134 is a WN6 with reported spectral variability on a period of $2.25 \mathrm{~d}$. Morel et al. (1999) extensively discuss two alternative origins, a compact companion or a rotationally modulated anisotropic outflow. They conclude that the latter hypothesis leads to better consistency with the observations. The $\mathrm{X}$-ray luminosity is rather low, and the radio spectral index (cf. (Dougherty \& Williams 2000) is thermal. Therefore we decide to keep WR 134 in our sample of single stars.

WR 134 belongs to the Cyg OB3 association, for which the distance has been revised from $D M=11.6 \mathrm{mag}$ to $11.2 \mathrm{mag}$ (Garmany \& Stencel 1992).

WR 136 shows, like WR 134, significant spectral variability. Koenigsberger et al. (1980) found a period of $4.5 \mathrm{~d}$ and concluded that the star might have a neutron star companion. Antokhin \& Cherepashchuk (1985) revised the period to $4.57 \mathrm{~d}$, while Robert et al. (1989) could not confirm the periodicity from polarization measurements. The X-ray luminosity is rather low, and the radio spectral index (cf. Dougherty \& Williams 2000) is thermal. Therefore we decide to keep WR 136 in our sample of single stars.

WR 136 belongs to the Cyg OB1 association, for which the distance has been drastically reduced by Garmany \& Stencel (1992) from $D M=11.3 \mathrm{mag}$ to $10.5 \mathrm{mag}$.

WR 138 is classified as WN5w + B? in the WR catalog. It is a long-period SB2 (Lamontagne et al. 1982: 1763 d; Annuk 1990: 1538 d). According to Lamontagne et al. (1982), both stars are of similar brightness. Our fits can perfectly reproduce the spectral energy distribution from UV to IR. However, the observed spectrum clearly shows absorption features at He I 4471 and He I 5876 that are obviously due to the OB companion. These features have equivalent widths as expected from a B0.5Ia star alone. Thus we cannot exclude that the visual flux is strongly contaminated by the companion. Therefore we exclude WR 138 from our sample of analyzed single-star spectra.

WR 138 also shows short-term variability, which has been attributed to a close compact companion (period $2.33 \mathrm{~d}$ ), making it a triple system (Lamontagne et al. 1982). However, such line profile variations are more likely due to the stellar wind dynamics. The X-ray flux of WR 138 is strong for a single WN star, but lies on the usual $L_{\mathrm{X}}-L_{\mathrm{bol}}$-relation for single $\mathrm{O}$ stars (Oskinova 2005).

The visual companion mentioned in the WR catalog is at $0.865^{\prime \prime}$ separation and about 2.87 HIPPARCOS magnitudes fainter and should not confuse the observations.

WR 139, better known as V444 Cyg, is a WN5 + O6III-V spectroscopic (SB2) and eclipsing binary with a period of $4.21 \mathrm{~d}$. The components are about equally bright (Hamann \& Schwarz 1992; Cherepashchuk et al. 1995), so we must exclude WR 139 from our single-star analyses.

WR 141 is classified as WN5w + O5V-III in the WR catalog. This SB2 system has a period of $21.7 \mathrm{~d}$ (Marchenko et al. 1998a). The O star contributes $34 \%$ to the total visual brightness (Lamontagne et al. 1996). Therefore we must exclude WR 141 from our single-star analyses.

WR 147 is classified as WN8(h) + B0.5V in the WR catalog. This extremely-long period binary has a period of $2880 \mathrm{~d}$ and was spatially resolved with HST by Lepine et al. (2001), who estimated the companion's spectral type as O5-7I-II(f). A nonthermal radio source $0.6^{\prime \prime}$ north of the the WR star is attributed to the colliding-wind interaction zone (Watson et al. 2002).

The WR star is 2.16 mag brighter than the companion (Niemela et al. 1998), which means that the OB star contributes only $12 \%$ to the visual flux. We neglect this contribution when analyzing the spectrum. As the separation between the two components is too wide for a strong evolutionary interaction, we also keep the star in our sample when comparing it with single-star evolutionary tracks.

WR 148 is classified as WN8h + B3IV/BH in the WR catalog. The radial velocity variations show a period of $4.317 \mathrm{~d}$ (Moffat \& Seggewiss 1980; Drissen et al. 1986). Both prefer the interpretation that the companion is a compact star or black hole. The large galactic height and system velocity supports the scenario of a runaway after a supernova kick. Panov et al. (2000) also found flare-like events and weak X-ray emission, attributed to accretion processes on the companion. In any case, additional light sources are 3 mag fainter than the stellar spectrum. Therefore we keep the star in our spectral analyses, but omit it in our comparison with single-star evolutionary tracks.

WR 151 is classified as WN4 + O5V in the WR catalog. This SB2 system has a period of $2.1 \mathrm{~d}$, and the components are about equally bright in the visual (Massey \& Conti 1981). Therefore we must omit this star from our spectral analysis.

WR 152 The hydrogen abundance and $v_{\infty}$ in Table 2 are taken from the analysis by Crowther et al. (1995d). This star belongs to the Cep OB1 association for which the distance has been revised from $D M=12.7$ mag to $12.2 \mathrm{mag}$ (Garmany \& Stencel 1992).

WR 155, also known as CQ Cep, is classified as WN6 + O9II-Ib in the WR catalog. This double-lined (SB2) spectroscopic binary has a period of $1.64 \mathrm{~d}$. Demicran et al. (1997) found that the components are about equally bright. Hamann \& Gräfener (2006) demonstrated that a slightly brighter companion is needed to model the observed SED. Thus we must exclude the composite spectrum of WR 155 from our single-star analyses.

WR 156 is classified as WN8h+OB? in the WR catalog, obviously because it is suspected to show "diluted emission lines". However, we can match the spectrum with our models perfectly. Lamontagne et al. (1983) found no radial velocity variations and consider the photometric variability as intrinsic. We conclude that this star is probably single. The hydrogen abundance $(27 \%$ by mass) is taken from Crowther et al. (1995b), while our Paper I gives a slightly higher value $(30 \%)$.

WR 157 is classified as WN5 + B1II binary. Turner et al. (1983) found a visual $B$ companion at 1 arcsec separation, which seems to be the brighter component. Therefore the star is omitted from our analyzed sample.

WR 158 is classified as WN7h + Be? in the WR catalog, because Andrillat \& Vreux (1992) found an O I 8446 line indicative of Be emission line stars. However, they also offer an alternative explanation by "non-standard environmental interaction". Moreover, WR 158 was suspected of being a binary because of the weakness ("dilution") of the emission lines. As our models can reproduce the observed spectrum, we conclude that there is no sufficient evidence of binarity. 


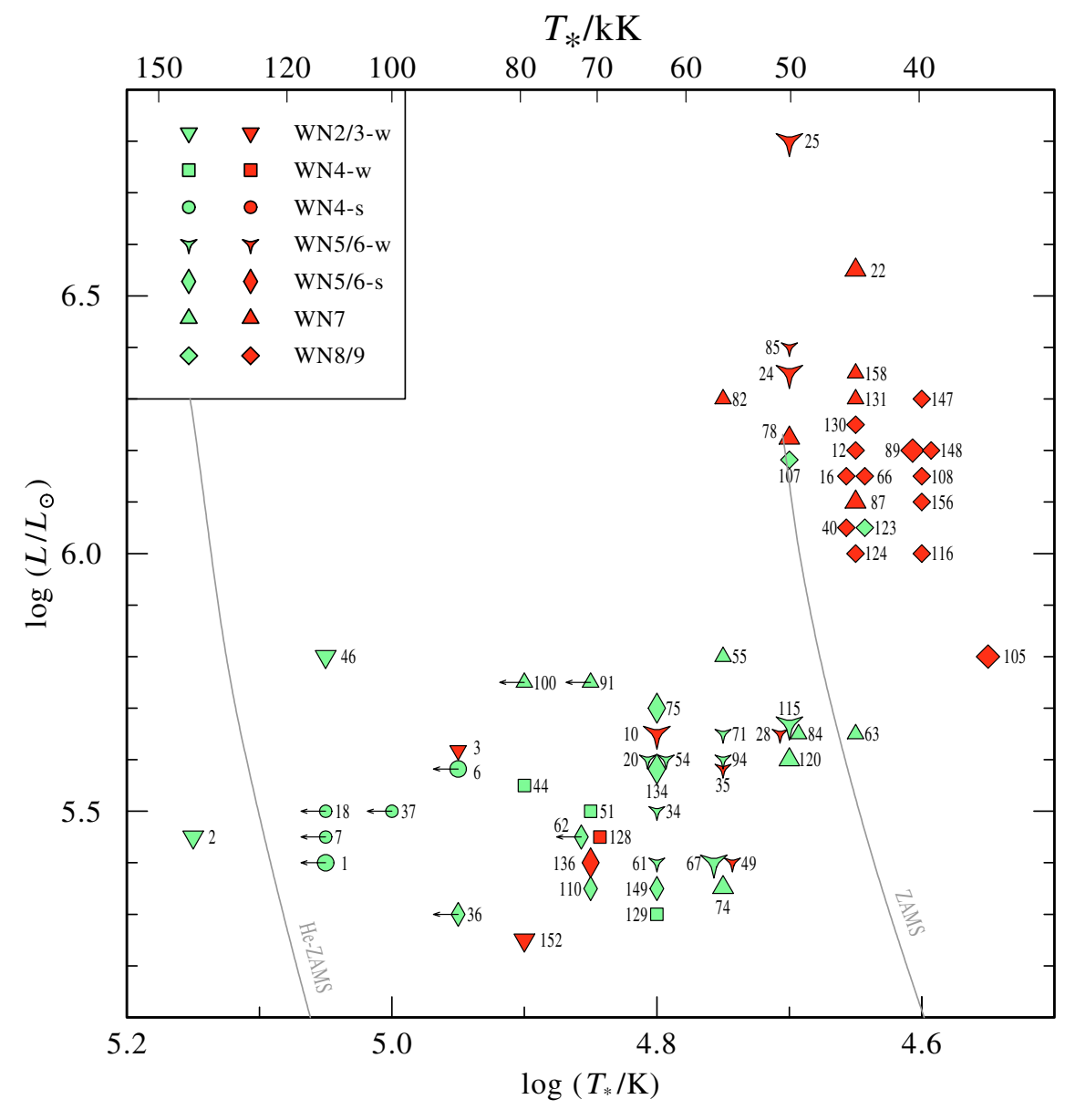

Fig. 8. Hertzprung-Russell diagram of the Galactic WN stars. The bigger symbols refer to stars whose distances are known from cluster/association membership. The filling color reflects the surface composition (dark/red: with hydrogen; light/green: hydrogen-free). The symbol shapes are coding for the spectral subtype (see inlet). A little arrow indicates that this particular star might be actually hotter because of the parameter degeneracy discussed in Sect. 4.1.

\section{The evolution of massive stars}

Figure 8 shows the Hertzsprung-Russell diagram of our program stars. The domains of the two spectral subclasses are clearly separated. Strikingly, the dividing line is just the hydrogen zero age main sequence (ZAMS): while the WNL stars lie to the cooler side of the ZAMS, the WNE stars populate a region between the hydrogen ZAMS and the helium main sequence (He-ZAMS).

The WNL stars, generally showing a significant hydrogen abundance, have stellar temperatures between 40 and $55 \mathrm{kK}$ and are very luminous (the arithmetic mean of $\log L / L_{\odot}$ is $6.22 \pm 0.20$ ). Compared to Paper I, this class has become significantly more luminous (by about 0.3 dex in the mean $\log L$ ). The reason is a combination of effects: higher stellar temperature, higher or anomalous reddening, larger cluster distances. The subtype calibration alone has only limited influence; adopting $M_{v}=-6.91 \mathrm{mag}$ as the typical WNL brightness would shift the WNLs with unknown distance (small symbols in Fig. 8) by 0.12 dex to lower luminosities.

The WNE stars, generally free of hydrogen, populate a range of lower luminosities. Their average $\left(\log L / L_{\odot}=5.5\right)$ is not revised compared to Paper I. The range in $\log L$ became more narrow, as several WNE-s stars that were considered to be very luminous in Paper I, such as WR 134 and WR 136, became less luminous in the present paper because their cluster distances have been revised downward.

The main objective for analyzing WR stars is to understand the evolution of massive stars. At the time of Paper I, the most advanced stellar evolution models were from Schaller et al. (1992). These tracks were not in good agreement with the empirical HRD of Paper I.
The empirical HRD of the WN, as obtained with the upgraded analyses presented here, is now the basis for re-assessing the question whether the evolution of massive stars is theoretically understood. In the meantime, since Paper I, the Geneva group has further improved the evolutionary models with upto-date physics and input data. Most important, the new tracks now come in a version that accounts for the effects of rotation (Meynet \& Maeder 2003). In Figs. 9 and 10 we plot the empirical HRD (now omitting the four confirmed close binaries) together with these evolutionary tracks for "typical" rotation (initial $v_{\text {rot }}=$ $300 \mathrm{~km} \mathrm{~s}^{-1}$ ) and for zero rotation, respectively. The metallicity is solar $(Z=0.02)$. By using different drawing styles, sections of the tracks are assigned to the spectral classes according to their surface composition: the WNL stage is reached when the star becomes hydrogen-deficient $\left(X_{\mathrm{H}}<0.40\right)$ and hotter than $20 \mathrm{kK}$; if hydrogen drops below $X_{\mathrm{H}}<0.05$, the star turns into a WNE type; the WC stage is reached when carbon appears at the surface $\left(X_{\mathrm{C}}>0.02\right)$.

The minimum mass for a star to reach any WR phase, which is $37 M_{\odot}$ without rotation, is reduced by rotation to $22 M_{\odot}$. While the track for $25 M_{\odot}$ initial mass ends with the supernova explosion as a red supergiant in case of no rotation, the corresponding track returns to the blue when "typical" rotation is included in the evolutionary model. Hence only the tracks with rotation can produce stars with the low luminosities observed in the WNE subclass. However, the WNE surface composition is only reached close to the helium main sequence, while observed WNE stars are scattered over cooler temperatures.

The HRD region where the WNL stars are observed is crossed by corresponding tracks for both versions, with and 


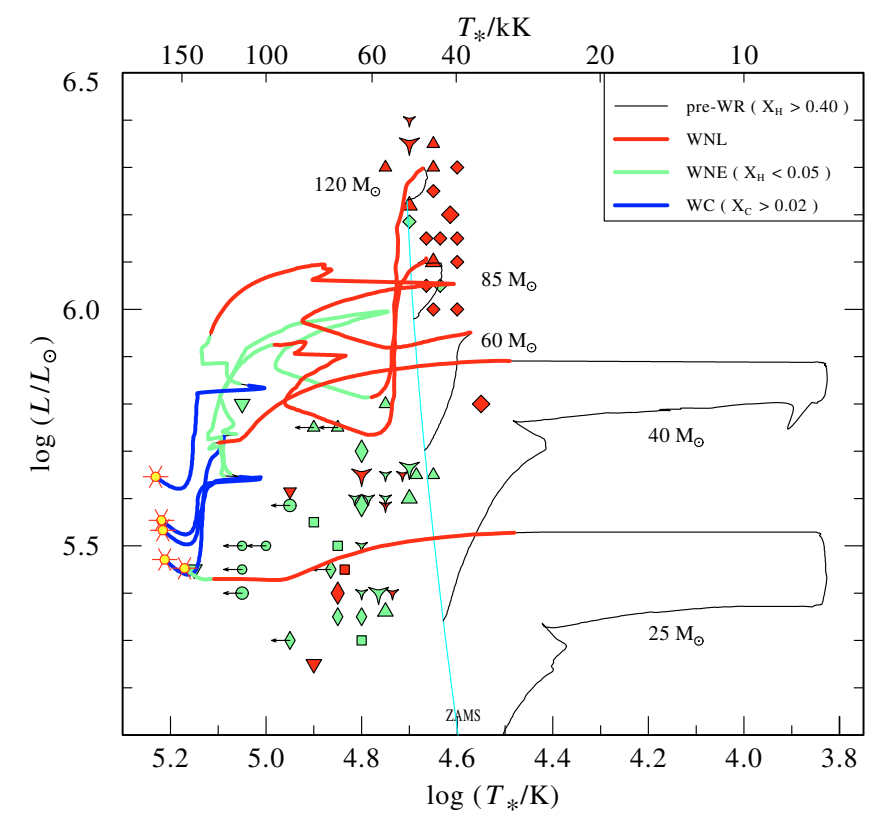

Fig. 9. Hertzprung-Russell diagram with the analyzed WN stars, now omitting the close binaries. The symbols have the same meaning as in Fig. 8. The evolutionary tracks from Meynet \& Maeder (2003) account for the effects of rotation (labels: initial mass). Thick lines refer to the different WR phases (see inlet).

without rotation. Very massive stars are predicted to skip the LBV excursions when rotation is included.

We want to recall that the evolutionary models we employ here do not account for magnetic fields. A dynamo mechanism ("Tayler-Spruit dynamo") has been proposed for massive stars, although there is no empirical proof so far for its existence. Maeder \& Meynet (2005) expect significant effects of the predicted field on the stellar evolution, but corresponding sets of tracks are not yet available.

Comparing the empirical HRD just with tracks can be misleading, as lifetimes in different parts of the tracks might be very different. Our sample comprises almost all WN stars from the earlier (the 6th) catalog of Galactic WR stars (van der Hucht et al. 1981). Those WN stars that have been added later to the recent WR catalog are usually highly reddened (and mainly discovered in the IR). Hence we can assume that our analyzed sample of WN stars is roughly complete for a well-defined part of our Galaxy, namely that part that can be observed with little interstellar absorption. We do not support the speculation by Massey (2003) that the number of WN stars is underestimated.

Therefore we use the Geneva tracks to generate synthetic populations, which we can compare with the empirical HRD. We adopt the usual power law for the initial mass function (IMF) with exponent $\beta\left(\mathrm{d} N=M^{-\beta-1} \mathrm{~d} M\right)$. The widely accepted value for this exponent is $\beta=1.35$ ("Salpeter IMF"), but there are indications that for massive stars in the Galactic field this exponent is somewhat higher, $\beta \gtrsim 1.8$ (Kroupa \& Weidner 2003). The star formation rate is set constant; this is only a rough approximation, as the analyzed sample contains several groups of stars that belong to the same association and are therefore probably coeval, which might not average out over the whole sample.

Now age and initial mass of a star are randomly chosen. Unfortunately, the available tracks are widely spaced in mass and qualitatively too different for a full interpolation. Therefore we apply each track as it is for a corresponding mass bin (borders are at $22,32,55,75,100,135 M_{\odot}$; for the track without

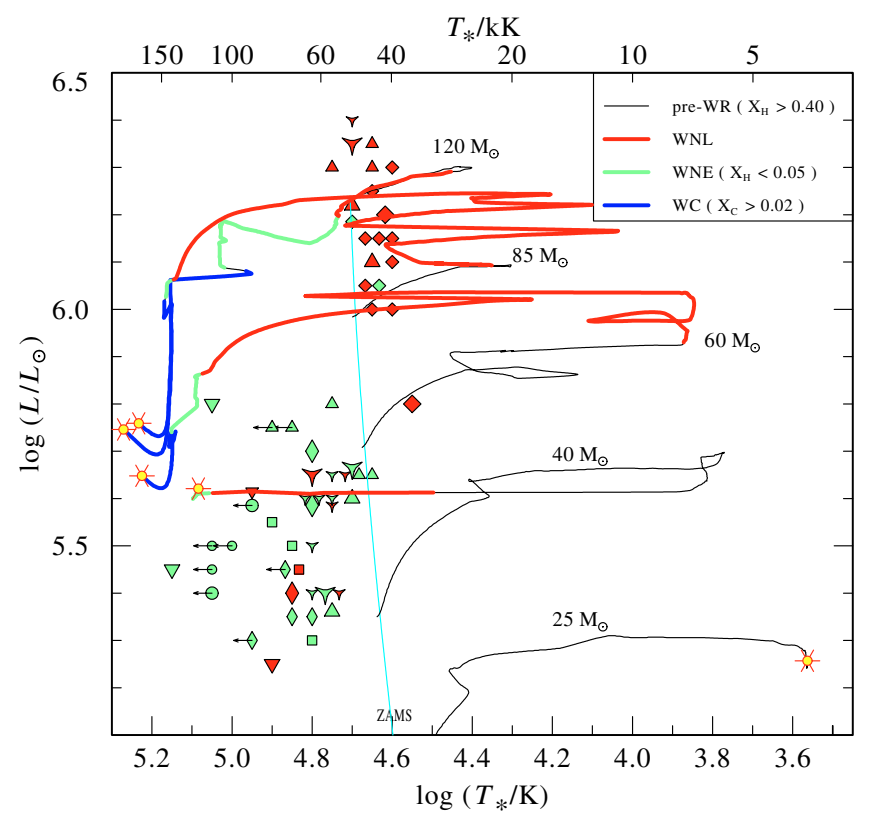

Fig. 10. Hertzprung-Russell diagram with the analyzed WN stars, now omitting the close binaries. The symbols have the same meaning as in Fig. 8. This set of evolutionary tracks from Meynet \& Maeder (2003) is calculated for zero rotation (labels: initial mass). Thick lines refer to the different WR phases (see inlet).

rotation, the border between the first and the second bin is set at $37 M_{\odot}$ ). Only the luminosity is scaled as $L \propto M_{\text {ini }}^{1.2}$ within each mass bin. The synthetic stars are finally assigned to a spectral class according to their surface composition, using the same criteria as described above when we plotted the tracks in different styles.

For the synthetic populations shown in Fig. 11 we created as many WN stars as our analyzed sample comprises (i.e. 59, without the proven close binaries). As the most conventional choice, we first take the tracks with rotation and adopt a "Salpeter IMF" (i.e. $\beta=1.35$ ). The result, shown in the top-right panel of Fig. 11, is disappointing when compared to the empirical HRD (topleft panel). Although the $25 M_{\odot}$ track produces WN stars that are as low-luminous as observed for the WNE, those synthetic low-luminosity WN stars are almost all of subtype WNL, i.e. they show hydrogen at their surface. Their predicted $X_{\mathrm{H}}$ is even higher than for most of the WNL stars.

Note that the observed sample contains more WNE than WNL stars. Taking the HRD positions, we find 39 WNE stars left of the ZAMS and 20 WNL stars on the right. On the basis of the hydrogen abundances, we have $33 \mathrm{WNE}$ and $26 \mathrm{WNL}$. In contrast, the number of WNE (i.e. nearly hydrogen-free) stars in the synthetic sample is only 10 . Hence the evolution predicts that less than one fifth of the WN stars are WNE, while more than half of the WN stars are actually observed to be hydrogen-free.

The stellar temperature of the WNE stars is another problem. In the evolutionary tracks the hydrogen-free surface appears only when the star has almost reached the helium main sequence. Observed WNE stars are mostly cooler, even when taking into account that some of the stars fall into the domain of parameter degeneracy. The other WNE stars have typically thin winds, i.e. the model continuum is formed at layers of low expansion velocity. The "stellar temperature" of such stars does not depend much on the definition of the reference radius; i.e. $T_{\text {eff }}$ at $\tau=2 / 3$ is roughly equal to our $T_{*}$ at $\tau=20$, so we conclude that these stars have a larger photospheric radius than predicted 


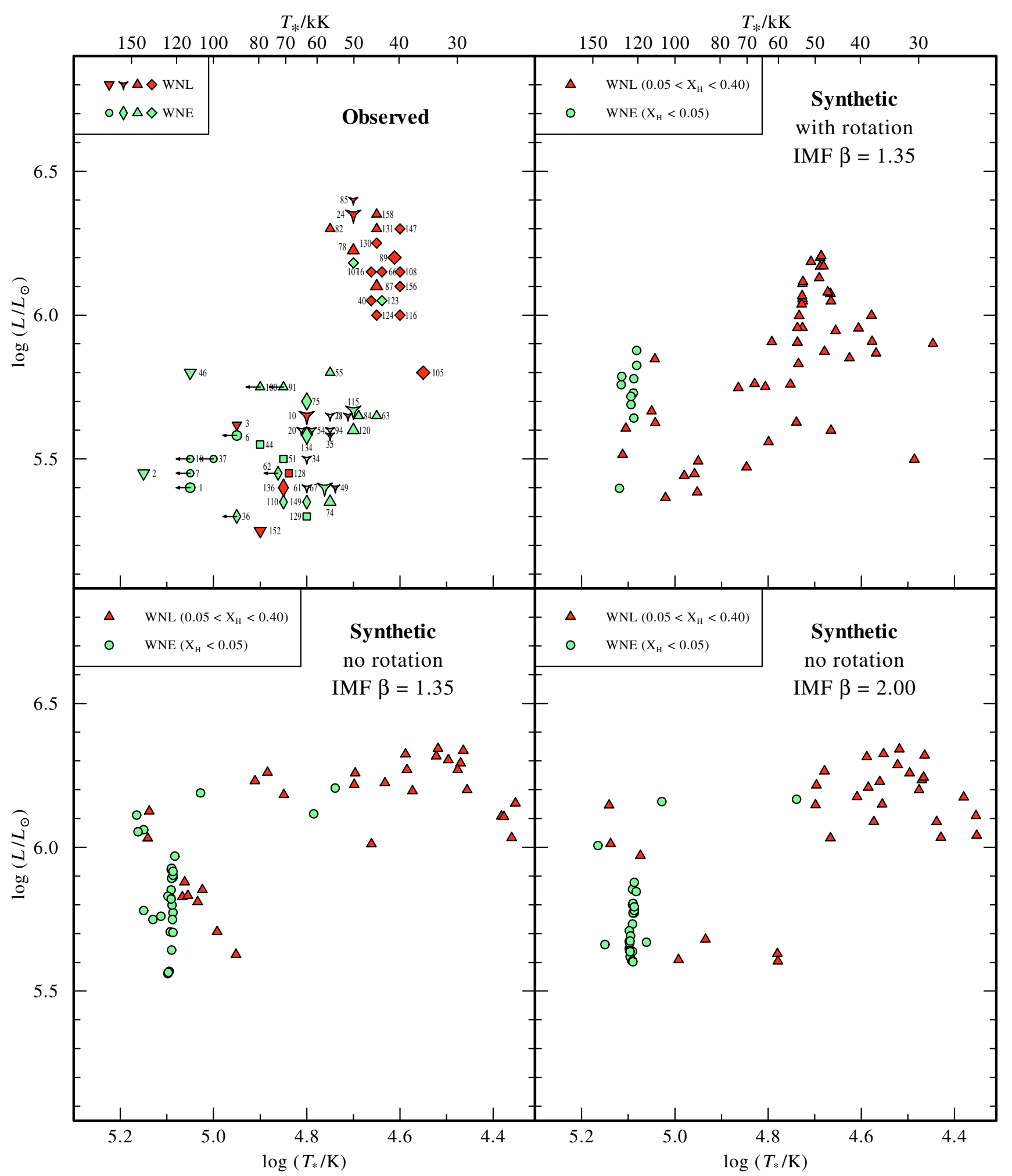

Fig. 11. Comparison of the Galactic WN stars with synthetic populations. Top-left panel: HRD of the analyzed Galactic WN stars (see Fig. 8 for the meaning of the symbols). Close binaries are omitted. Other panels: synthetic WN population, based on the Geneva tracks (Meynet \& Maeder 2003). The filling color reflects the surface composition (dark/red: with hydrogen; light/green: almost hydrogen-free). The synthetic populations are for different assumptions as indicated in the plots. Top-right panel: evolution with rotation, Salpeter IMF ( $\beta=1.35)$; bottom-left panel: evolution without rotation, Salpeter IMF ( $\beta=1.35)$; bottom-right panel: evolution without rotation and "steep" IMF $(\beta=2.00)$.

by the stellar models. A speculative explanation is that there is a huge extended layer on top of the "real" hydrostatic core that expands only slowly (subsonic velocities), possibly driven by the "hot iron bump" in the mean opacity.
The WNL group is also not reproduced well by the synthetic population, because their typical luminosity is smaller than observed. No synthetic star lies above $\log L / L_{\odot}=6.2$, while the empirical HRD shows eight such stars of very high luminosity. 
Two of these stars have a known distance (WR 24 and WR 78), while the others rely on the subtype calibration. Even with this caveat, there seems to be a discrepancy. A flatter IMF (e.g. $\beta=$ 0.35 ) would produce more WNL stars at highest luminosities.

After finding so many discrepancies, one might wonder if the tracks without rotation really lead to even larger disagreement with the empirical HRD. In the lower-left panel of Fig. 11 we display the corresponding synthetic population, again for the Salpeter IMF. As expected, the low-luminous WNE stars are missing. Interestingly, the luminous WNL group in this plot reproduces the high average luminosity of the observed WNL sample nicely, and only the scatter in temperature is larger. Remarkable is also the statistical distribution between WNE and WNL, which is now 30:29, i.e. close the observed slight WNE majority. With a steeper IMF, $\beta=2.0$, the dichotomy between the WNE and WNL becomes even nicer, while their number ratio is not affected. Only the temperature scatter of the WNE is of course also not reproduced by the synthetic WNE, which are all sitting near the He-ZAMS. Hence we must conclude that, based on the shown comparison between the analyzed Galactic WN sample and the synthetic populations, there is no preference for the tracks that account for the stellar rotation.

One can discuss more statistical numbers that are predicted by the evolutionary calculations. The ratio between the number of WC to WN stars in the WR catalog is about 0.9 , while the population synthesis yields only $17 \mathrm{WC}$ stars with rotation and $\beta=1.35$, but 45 and $37 \mathrm{WC}$ stars without rotation and $\beta=1.35$ and 2.00, respectively (always compared to $59 \mathrm{WN}$ stars). Thus the non-rotating models are again closer to the observation.

However, Meynet \& Maeder (2003) point out that the low WC-to-WN ratio predicted by the models with rotation fits better into the trend with metallicity when different galaxies are compared. On the other hand, Eldridge \& Vink (2006) show that this trend can also be explained without rotation effects, when the WR mass-loss rates depend on metallicity. Such dependence is theoretically predicted from theoretical models for radiationdriven WR winds (Vink \& de Koter 2005; Gräfener \& Hamann 2006 and in prep.). Thus it seems that the WC/WN metallicity trend does not unambiguously support the evolutionary models with rotation.

The number of Galactic WR to $\mathrm{O}$ stars, about 0.1 , is better reproduced by the rotating than by the non-rotating models (Meynet \& Maeder 2003). However, the question of incompleteness might be more severe for the number of $\mathrm{O}$ stars than for the WR stars.

Summarizing the comparison between the results of our spectral analyses of the Galactic WN stars and the predictions of the Geneva evolutionary calculations, we conclude that there is rough qualitative agreement. However, the quantitative discrepancies are still severe, and there is no preference for the tracks that account for the effects of rotation. We wonder how future evolutionary models that account for magnetic fields will compare with our empirical HRD. Based on the presently existing tracks, it seems that the evolution of massive stars is still not satisfactorily understood.

Concluding remark. In this paper we presented quantitative spectral analyses of the Galactic WN stars, based on the lineblanketed Potsdam Wolf-Rayet (PoWR) model atmospheres. We hope that the obtained stellar and atmospheric parameters provide an empirical basis for various studies about the origin, evolution, and physics of the Wolf-Rayet stars and their powerful winds.
Acknowledgements. We thank L. Oskinova for many useful discussions and careful reading of the manuscript. We also acknowledge the helpful suggestions of the referee, J. Vink.

\section{References}

Andrillat, Y., \& Vreux, J.-M. 1992, A\&A, 253, 37

Annuk, K. 1990, Acta Astronomica, 40, 267

Antokhin, I. I., \& Cherapashchuk, A. M. 1985, Soviet Astron. Lett., 11, 355

Antokhin, I. I., Bertrand, J.-F., Lamontagne, R., \& Moffat, A. F. J. 1995, IAUS, 163,62

Arnal, E. M., \& Roger, R. S. 1997, MNRAS, 285, 253

Balona, L. A., Egan, J., \& Marang, F. 1989, MNRAS, 240, 103

Barniske, A., Oskinova, L., Hamann, W.-R., \& Gräfener, G. 2006, in Stellar Evolution at Low Metallicity: Mass Loss, Explosions, Cosmology. ASP Conf. Ser. (in press)

Cardelli, J. A., Clayton, G. C., \& Mathis, J. S. 1989, ApJ, 345, 245

Cherepashchuk, A. M., Koenigsberger, G., Marchenko, S. V., \& Moffat, A. F. J. 1995, A\&A, 293, 142

Crowther, P. A., Hillier, D. J., \& Smith, L. J. 1995a, A\&A, 293, 172

Crowther, P. A., Hillier, D. J., \& Smith, L. J. 1995b, A\&A, 293, 403

Crowther, P. A., Smith, L. J., Hillier, D. J., \& Schmutz, W. 1995c, A\&A, 293, 427

Crowther, P. A., Smith, L. J., \& Hillier 1995d, A\&A, 302, 457

Crowther, P. A., Smith, L. J., \& Willis, A. J. 1995e, A\&A, 304, 269

Demicran, O., Ak, H., Özdemir, S., et al. 1997, AN, 318, 267

Drissen, L., Lamontagne, R., Moffat, A. F. J., Bastien, P., \& Seguin, M. 1986, ApJ, 304, 188

Drissen, L., Robert, C., \& Moffat, A. F. J. 1992, ApJ, 386, 288

Dougherty, S. M., \& Williams, P. M. 2000, MNRAS, 319, 1005

Eldridge, J. J., \& Vink, J. S. 2006, A\&A, in press

Fitzpatrick, E. L. 1999, PASP, 111, 63

Gamen, R., \& Niemela, V. S. 1999, Rev. Mex. Astron. Astrofis. Ser. Conf., 8, 55 Garmany, C. D., \& Stencel, R. E. 1992, A\&AS, 94, 211

Gräfener, G., \& Hamann, W.-R. 2002, A\&A, 432, 633

Gräfener, G., \& Hamann, W.-R. 2006, in Stellar Evolution at Low Metallicity: Mass Loss, Explosions, Cosmology, ed. H. J. G. L. M. Lamers, N. Langer, T. Nugis, \& K. Annuk, ASP Conf. Ser. (in press)

Gräfener, G., Koesterke, L., \& Hamann, W.-R. 2002, A\&A, 387, 244

Hamann, W.-R., \& Schwarz, E. 1992, A\&A, 261, 523

Hamann, W.-R., \& Gräfener, G. 2004, A\&A, 427, 697

Hamann, W.-R., \& Koesterke, L. 1998, A\&A, 333, 251 (Paper I)

Hamann, W.-R., \& Gräfener, G. 2006, in Massive Stars in Interacting Binaries, ed. N. St-Louis, \& A. F. J. Moffat, ASP Conf. Ser. (in press)

Hamann, W.-R., Koesterke, L., \& Wessolowski, U. 1995, A\&AS, 113, 459

Herald, J. E., Hillier, D. J., \& Schulte-Ladbeck, R. E. 2001, ApJ, 548, 932

Hillenbrand, L. A., Massey, P., Strom, S. E., \& Merrill, K. M. 1993, AJ, 106, 1906

Hillier, D. J., \& Miller, D. L. 1998, ApJ, 496, 407

van der Hucht, K. A. 2001, New Astron. Rev., 45, 135

van der Hucht, K. A., Conti, P. S., Lundström, I., \& Stenholm, B. 1981, Space Sci. Rev., 28, 227

Isserstedt, J., Moffat, A. F. J., \& Niemela, V. S. 1983, A\&A, 126, 183

Koenigsberger, G., Firmani, C., \& Bisiacchi, G. F. 1980, Rev. Mex. Astron. Astrofis., 5, 45

Kroupa, P., \& Weidner, C. 2003, ApJ, 598, 1076

Lamontagne, R., Moffat, A. F. J., Koenigsberger, G., \& Seggewiss, W. 1982, ApJ, 253, 230

Lamontagne, R., Moffat, A. F. J., \& Seggewiss, W. 1983, ApJ, 269, 596

Lamontagne, R., Moffat, A. F. J., Drissen, L., et al. 1996, AJ, 112, 2227

Langer, N. 1989, A\&A, 210, 93

Lepine, S., Wallace, D., Shara, M. M., et al. 2001, AJ, 122, 3407

Lortet, M.-C., Georgelin, Y. P., \& Georgelin, Y. M. 1987, A\&A, 180, 65

Lundstroem, I., \& Stenholm, B. 1984, A\&AS, 58, 163

Maeder, A., \& Meynet, G. 2005, A\&A, 440, 1041

Marchenko, S. V., \& Moffat, A. F. J. 1998, ApJ, 499, 195

Marchenko, S. V., Moffat, A. F. J., \& Eenens, P. R. J. 1998a, PASP, 110, 1416

Marchenko, S. V., Moffat, A. F. J., van der Hucht, K. A., et al. 1998b, A\&A, 331, 1022

Marchenko, S. V., Arias, J., Barba, R., et al. 2000, AJ, 120, 2101

Marchenko, S. V., Moffat, A. F. J., Crowther, P. A., et al. 2004, MNRAS, 353, 153

Massey, P. 2003, ARA\&A, 41, 15

Massey, P., \& Conti, P. S. 1981, ApJ, 244, 173

Massey, P., \& Johnson, J. 1993, AJ, 105, 980

Matthews, J. M., \& Moffat, A. F. J. 1994, A\&A, 283, 493

Meynet, G., \& Maeder, A. 2003, A\&A, 404, 975 
Moneti, A., Stolovy, S., Blommaert, J. A. D. L., Figer, D. F., \& Najarro, F. 2001, A\&A, 366, 106

Moffat, A. F. J., \& Seggewiss, W. 1980, A\&A, 86, 87

Moffat, A. F. J., \& Shara, M. M. 1986, AJ, 92, 952

Morel, T., St-Louis, N., \& Marchenko, S. V. 1997, ApJ, 482, 470

Morel, T., Marchenko, S. V., Eenens, P. R. J., et al. 1999, ApJ, 518, 428

Niemela, V. S. 1982, in IUA Sym., 99, 299

Niemela, V. S., \& Moffat, A. F. J. 1992, ApJ, 259, 213

Niemela, V. S., Barba, R. H., \& Shara, M. M. 1995, IAU Symp., 163, 245

Niemela, V. S., Shara, M. M., Wallace, D. J., et al. 1998, AJ, 115, 2047

Niemela, V. S., Gamen, R., Morrell, N. I., Benítez, S. G. 1999, IAU Symp., 193, 26

Nugis, T., \& Lamers, H. J. G. L. M. 2000, A\&A, 360, 227

Oskinova, L. M. 2005, MNRAS, 361, 679

Raassen, A. J. J., van der Hucht, K. A., Mewe, R., et al. 2003, A\&A, 402, 653

Rauw, G., Vreux, J.-M., Gosset, E., Manfroid, J., \& Niemela, V. S. 1996a, in Wolf-Rayet stars in the framework of stellar evolution. Liége, p. 303

Rauw, G., Vreux, J.-M., Gosset, E., Hutsemékers, D., Magain, P., \& Rochowicz, K. 1996b, A\&A, 306, 771

Robert, C., Moffat, A. F. J., Bastien, P., et al. 1989, ApJ, 347, 1034
Schaller, G., Schaerer, D., Meynet, G., \& Maeder, A. 1992, A\&AS, 96, 269 Schulte-Ladbeck, R. E. 1994, Ap\&SS, 221, 347

Schweickhardt, J., Schmutz, W., Stahl, O., Szeifert, Th., \& Wolf, B. 1999, A\&A, 347,127

Seaton, M. J. 1979, MNRAS, 187, 73

Smith, L. F. 1968, MNRAS, 140, 409

Smith, L. F., Shara, M. M., \& Moffat, A. F. J. 1996, MNRAS, 281, 163

Steiner, J. E., \& Diaz, M. P. 1998, PASP, 110, 276

Steiner, J. E., Cieslinski, D., \& Jablonski, F. J. 1988, ASPC, 1, 67

Steiner, J. E., Cieslinski, D., Jablonski, F. J., \& Williams, R. E. 1999, A\&A, 351, 1021

Tovmassian, H. M., Navarro, S. G., \& Cardona, O. 1996, AJ, 111, 306

Turner, D. G., Moffat, A. F. J., Lamontagne, R., \& Maitzen, H. M. 1983, AJ, 88, 1199

Underhill, A. B., \& Hill, G. M. 1994, ApJ, 432, 770

Veen, P. M., van Genderen, A. M., \& van der Hucht, K. A. 2002, A\&A, 385, 619

Vink, J. S., \& de Koter, A. 2005, A\&A, 442, 587

Watson, S. K., Davis, R. J., Williams, P. M., \& Bode, M. F. 2002, MNRAS, 334, 631 
W.-R. Hamann et al.: The Galactic WN stars, Online Material p 1

\section{Online Material}


The following figures display for each of our program stars the observed spectrum (blue) with a model (red). The upper panel, corresponding to Fig. 3, shows the spectral energy distribution (IUE low-resolution spectrum if available, visual and 2MASS photometry). The model SED was geometrically diluted according to the distance modulus $D M$ and reddened with $E_{b-v}$ as given in the figure. Keywords CARDELLI or FITZPATRICK indicate the applied reddening law (default: Seaton's law), the given parameter being $R_{V}$ (see Sect. 4.2).

Most models were taken from our PoWR model grids (see Hamann \& Gräfener 2004). The grid parameters $\operatorname{are} \log T_{*}$ and $\log R_{\mathrm{t}}$. Note that the grids were calculated for a fixed luminosity of $\log L / L_{\odot}=5.3$, and only scaled for the individual star; the scaling factor is indicated as the logarithmic "shift" in the plots. The so-called WNE grid is calculated for zero hydrogen and a terminal wind velocity of $1600 \mathrm{~km} \mathrm{~s}^{-1}$, while the models in the WNL grid have $20 \%$ hydrogen (by mass) and $v_{\infty}=1000 \mathrm{~km} \mathrm{~s}^{-1}$.

As the plotted models are mostly taken from the prepared grids (if not otherwise stated), their hydrogen abundance $X_{\mathrm{H}}$ and terminal velocity $v_{\infty}$ do generally not agree precisely with the final parameters of our analysis as given in Table 2 . 
W.-R. Hamann et al.: The Galactic WN stars, Online Material p 3

MODEL START 04/01/03 20:19:23 112202/0.3D/1600 L=5.3 N=1.5\% C=1E-4 Fe=1.4E-3 D4 WN-NODIEL 14-18 AFTER JOB NO.311
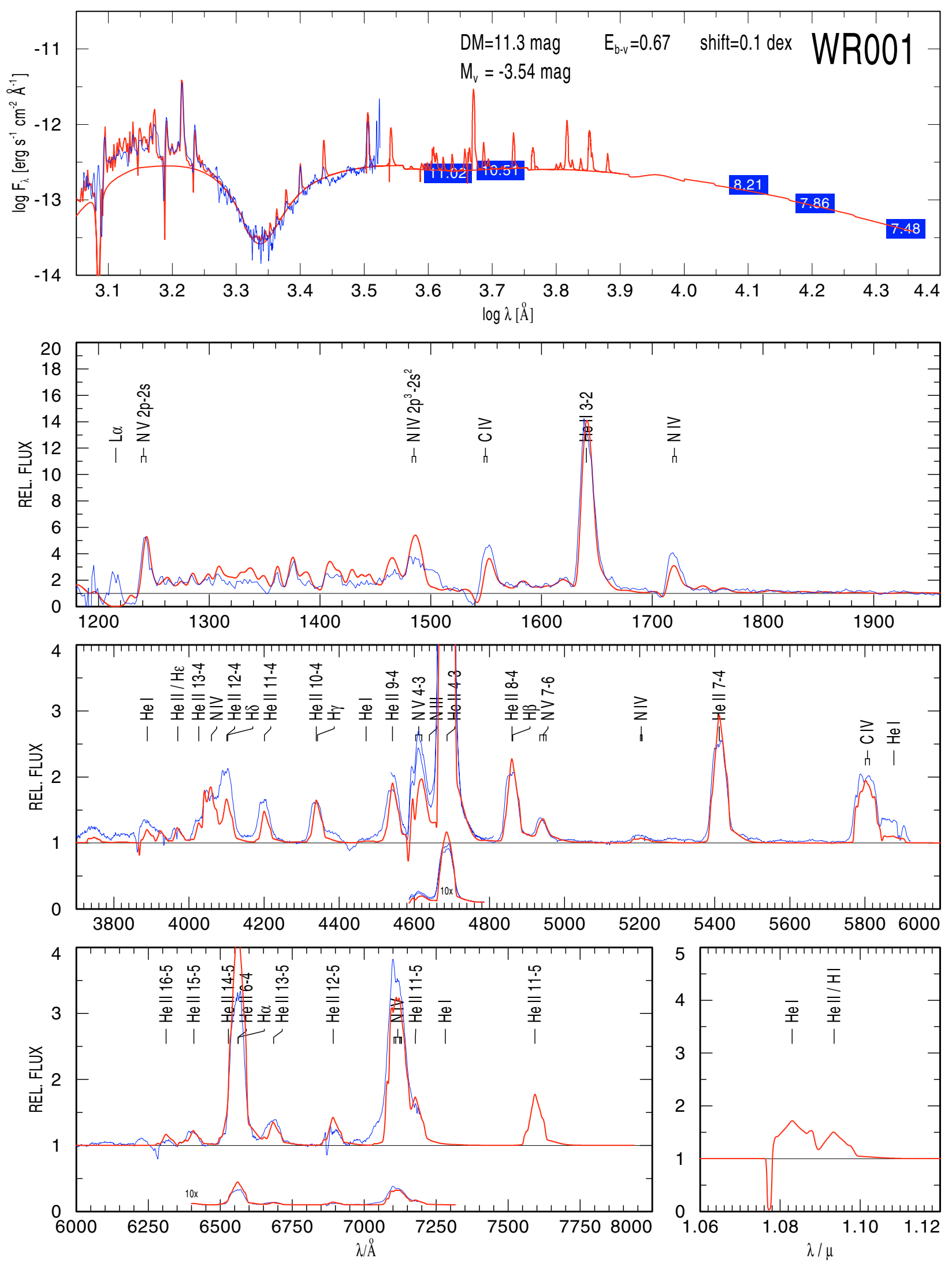

Fig. 12. Model: WNE 14-18, $T_{*}=112 \mathrm{kK}, \log \left(R_{\mathrm{t}} / R_{\odot}\right)=0.3$. 
W.-R. Hamann et al.: The Galactic WN stars, Online Material $p 4$

MODEL START 03/13/03 16:31:27 141254/0.5D/1600 L=5.3 N=1.5\% C=1E-4 Fe=1.4E-3 D4 WN-NODIEL 16-16 AFTER JOB NO.961
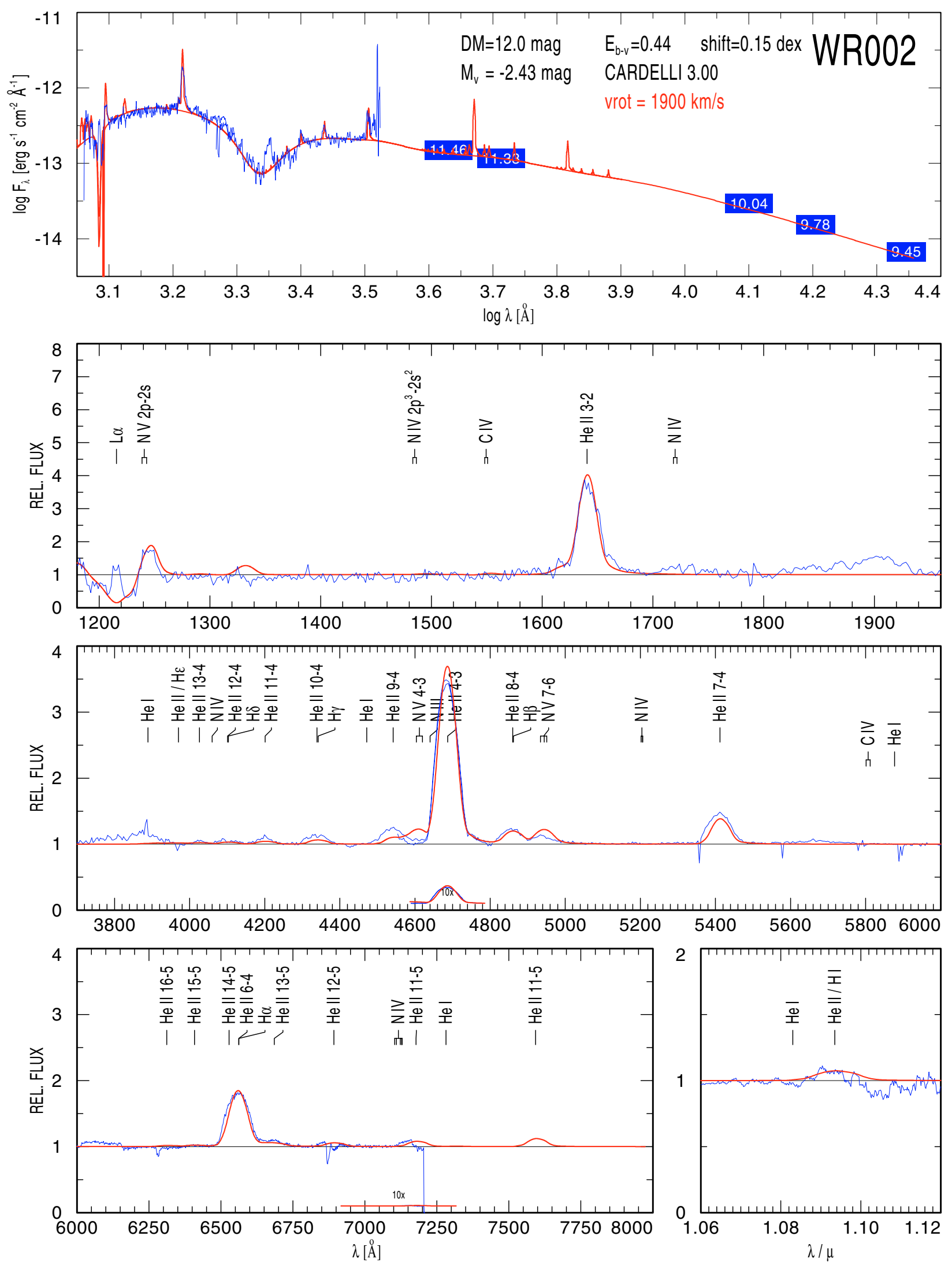

Fig. 13. Model: WNE 16-16, $T_{*}=141 \mathrm{kK}, \log \left(R_{\mathrm{t}} / R_{\odot}\right)=0.5$. 
W.-R. Hamann et al.: The Galactic WN stars, Online Material p 5

MODEL START 08/29/05 09:53:32 89125/1.2D/2700 L5.3 H20 N1.5\% C1E-4 Fe0.14\% D4 WNL-NODR 12-09 AFTER JOB NO.569
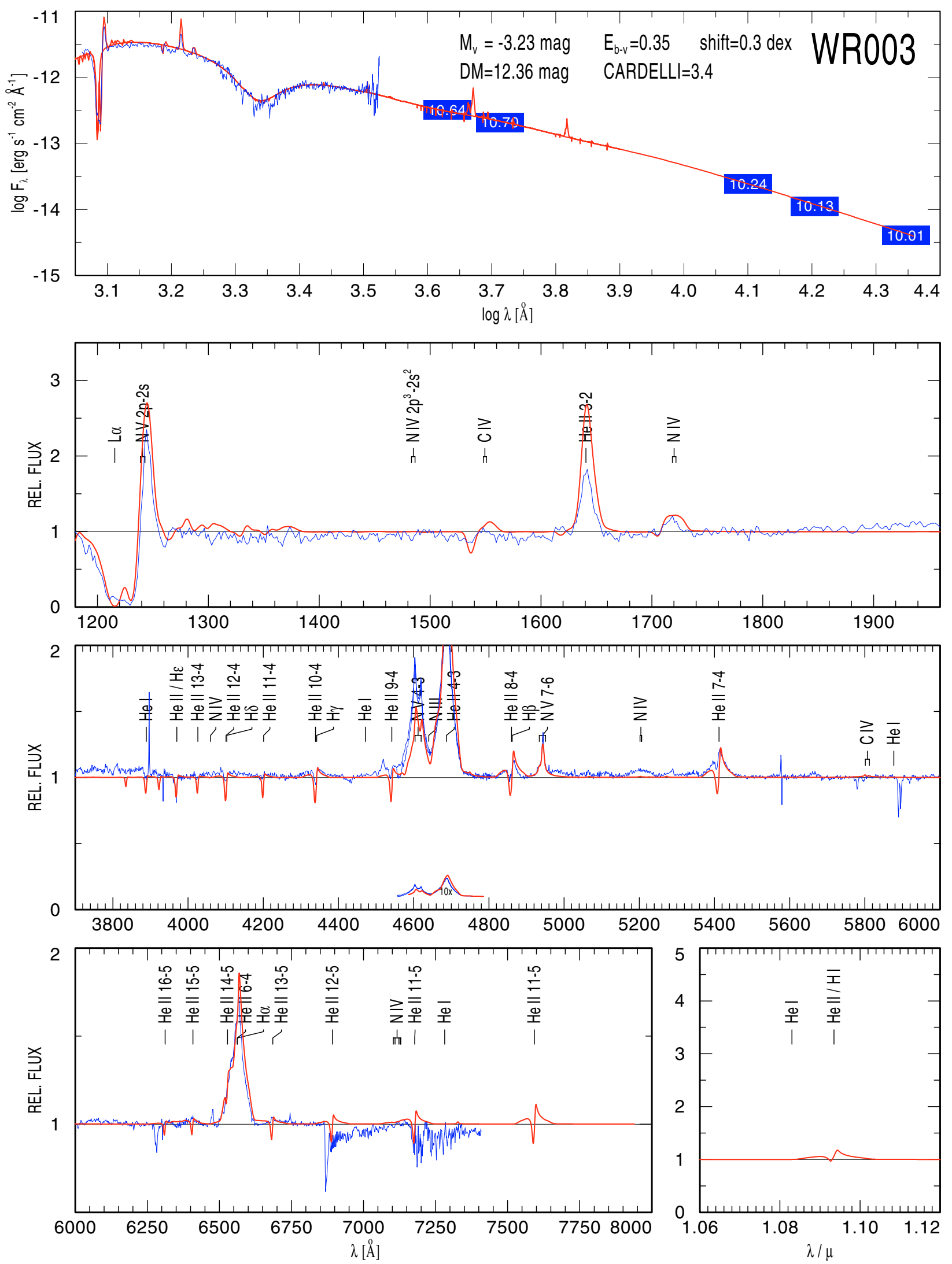

Fig. 14. Individual Model: WNL 12-09, $T_{*}=89 \mathrm{kK}, \log \left(R_{\mathrm{t}} / R_{\odot}\right)=1.2$ but $v_{\infty}=2700 \mathrm{~km} \mathrm{~s}^{-1}$. 
W.-R. Hamann et al.: The Galactic WN stars, Online Material p 6

MODEL START 03/08/03 04:38:11 89125/0.3D/1600 L=5.3 N=1.5\% C=1E-4 Fe=1.4E-3 D4 WN-NODIEL 12-18 AFTER JOB NO.185
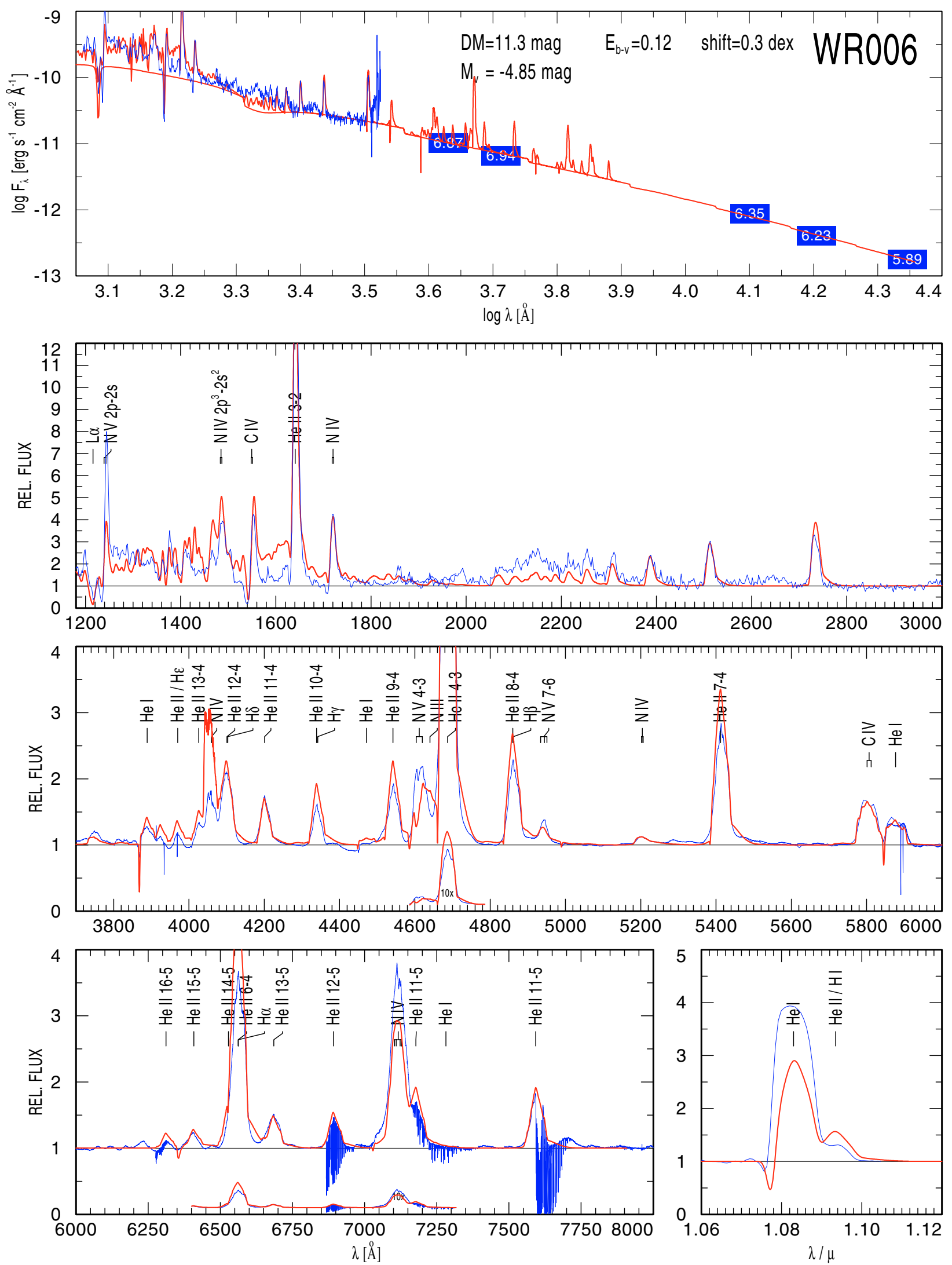

Fig. 15. Model: WNE $12-18, T_{*}=89 \mathrm{kK}, \log \left(R_{\mathrm{t}} / R_{\odot}\right)=0.3$. 
W.-R. Hamann et al.: The Galactic WN stars, Online Material $p 7$

MODEL START 04/01/03 20:19:23 112202/0.3D/1600 L=5.3 N=1.5\% C=1E-4 Fe=1.4E-3 D4 WN-NODIEL 14-18 AFTER JOB NO.311
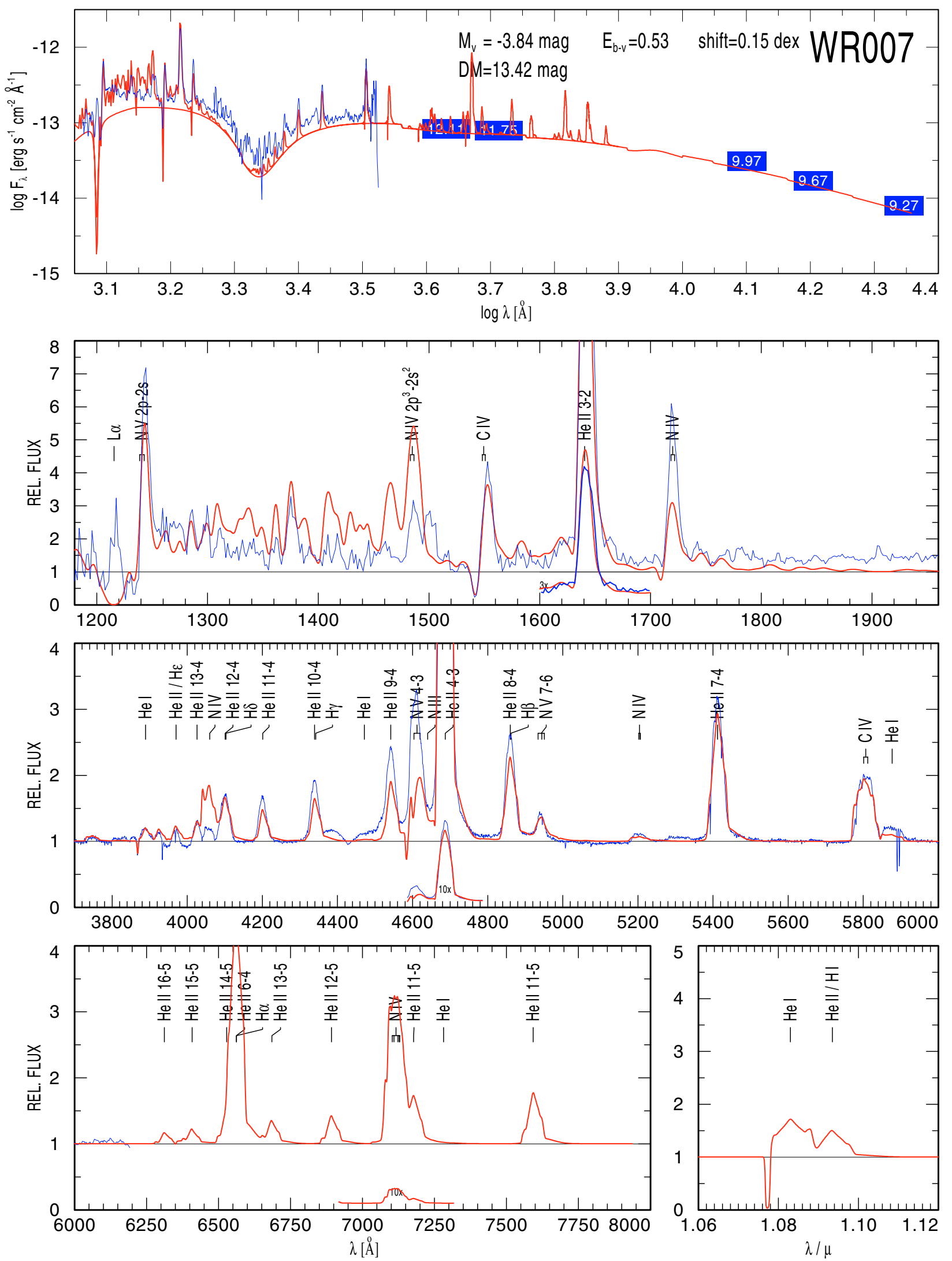

Fig. 16. Model: WNE 14-18, $T_{*}=112 \mathrm{kK}, \log \left(R_{\mathrm{t}} / R_{\odot}\right)=0.3$. 
W.-R. Hamann et al.: The Galactic WN stars, Online Material $p 8$

MODEL START 10/27/03 09:21:21 63096/1.2D/1000 L=5.3 H20 N1.5\% C1E-4 Fe0.14\% D4 WNL-NODR 09-09 AFTER JOB NO.625
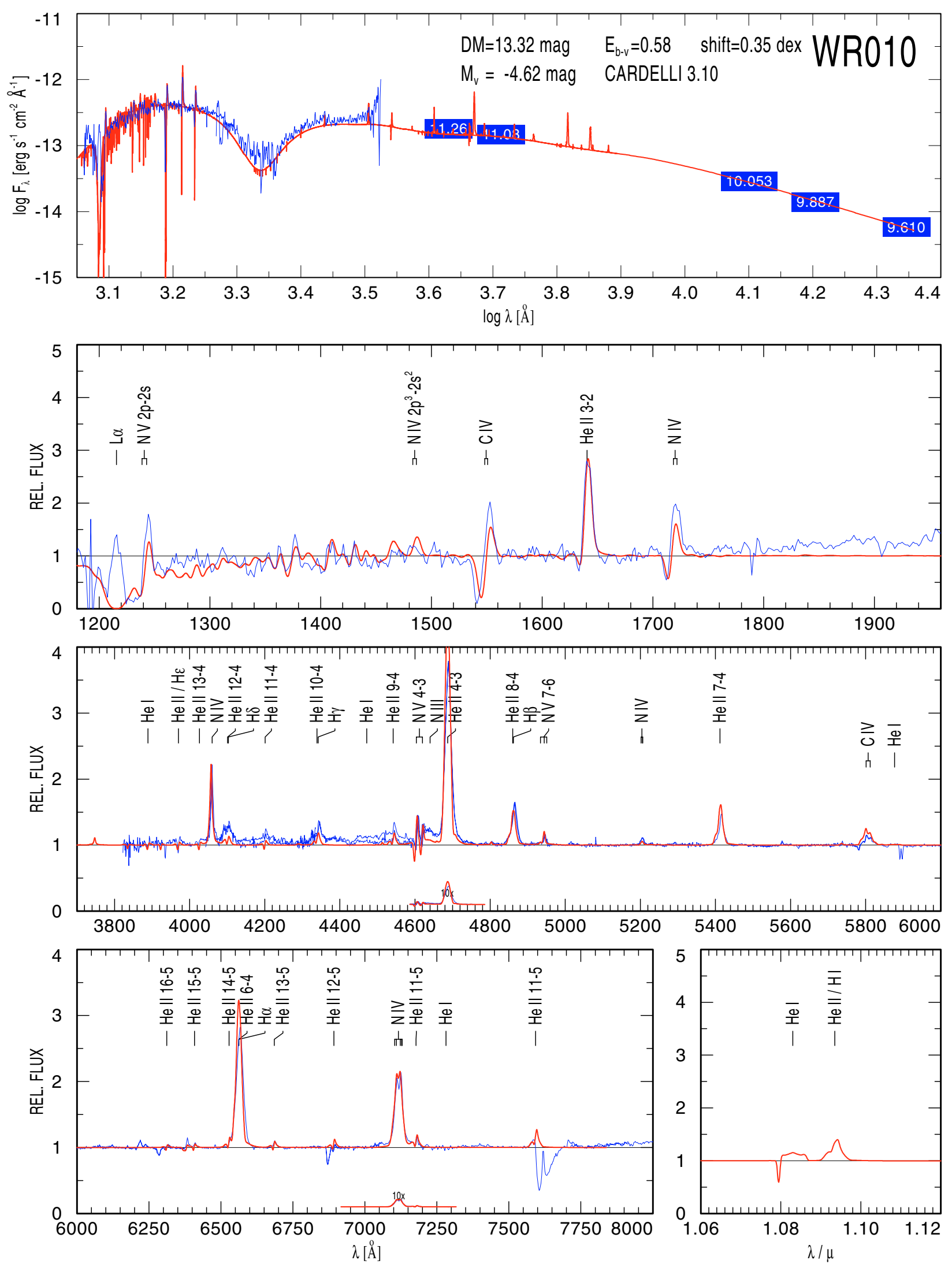

Fig. 17. Model: WNL 09-09, $T_{*}=63 \mathrm{kK}, \log \left(R_{\mathrm{t}} / R_{\odot}\right)=1.2$. 
W.-R. Hamann et al.: The Galactic WN stars, Online Material $p 9$

MODEL START 11/24/03 09:56:15 44668/1.0D/1000 L5.3 H20 N1.5\% C1E-4 Fe0.14\% D4 WNL-NODR 06-11 AFTER JOB NO.727
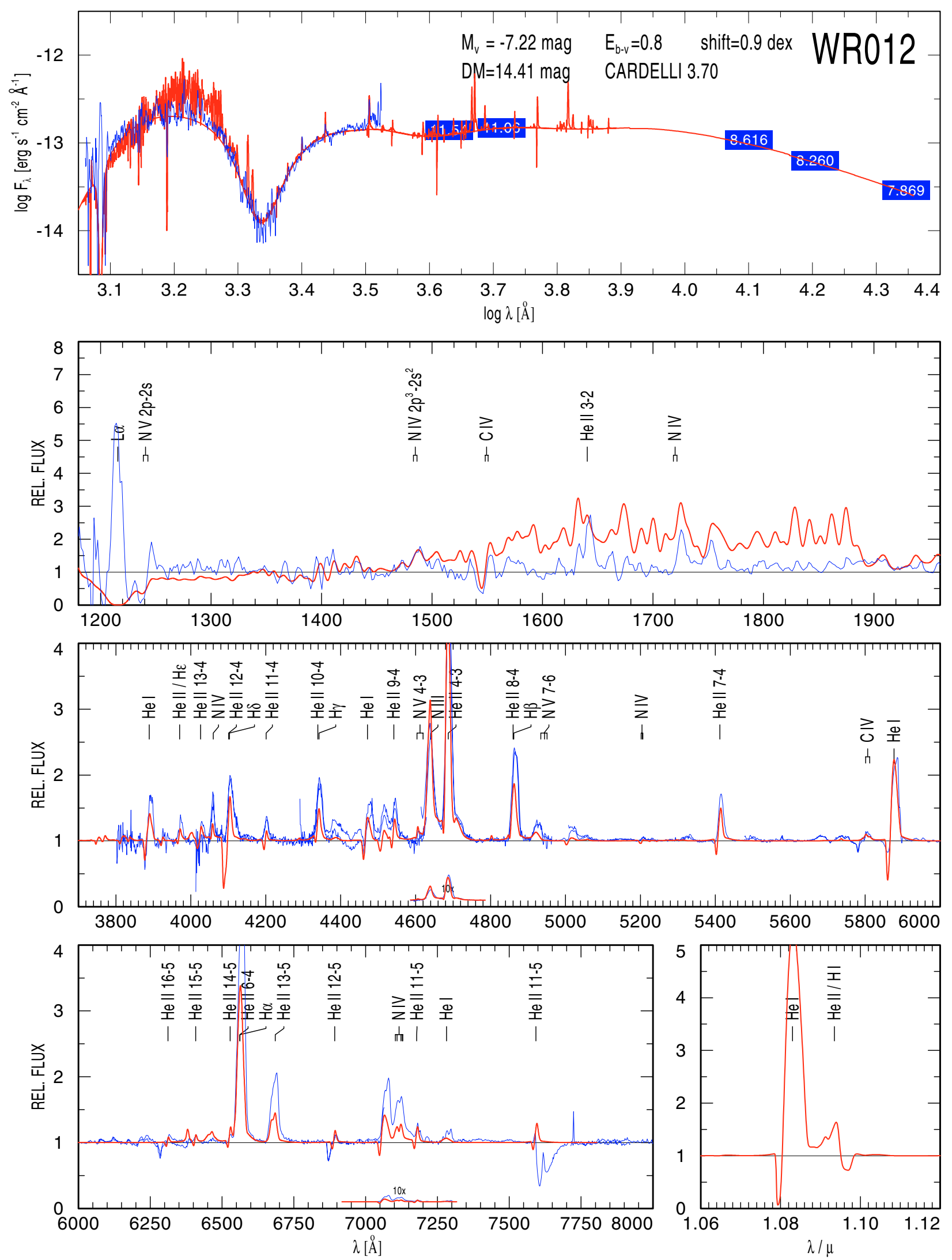

Fig. 18. Model: WNL 06-11, $T_{*}=45 \mathrm{kK}, \log \left(R_{\mathrm{t}} / R_{\odot}\right)=1.0$. 
W.-R. Hamann et al.: The Galactic WN stars, Online Material p 10

MODEL START 11/25/03 12:51:17 44668/0.9D/1000 L5.3 H20 N1.5\% C1E-4 Fe0.14\% D4 WNL-NODR 06-12 AFTER JOB N0.667
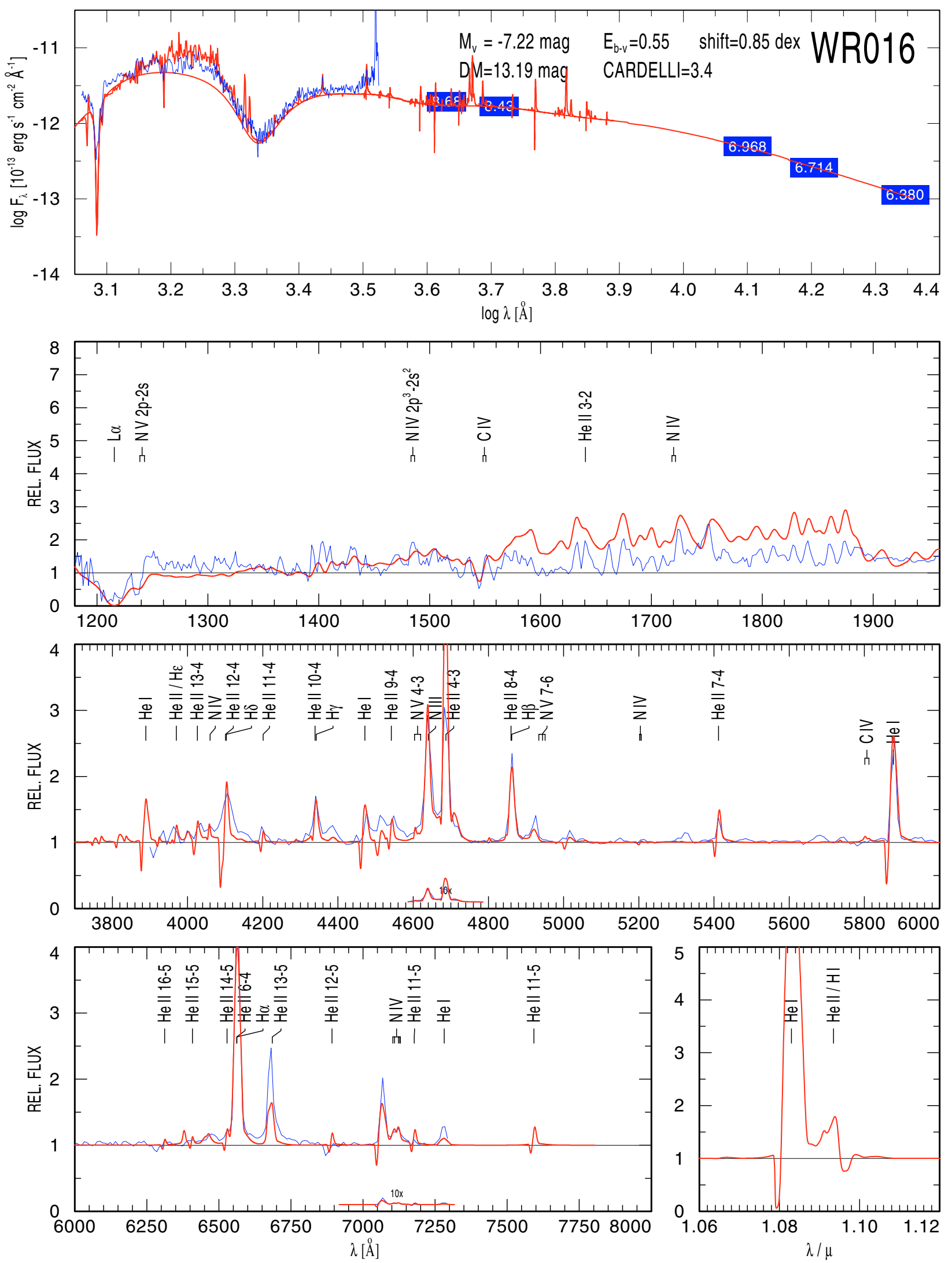

Fig. 19. Model: WNL 06-12, $T_{*}=45 \mathrm{kK}, \log \left(R_{\mathrm{t}} / R_{\odot}\right)=0.9$. 
W.-R. Hamann et al.: The Galactic WN stars, Online Material p 11

MODEL START 04/01/03 20:19:23 112202/0.3D/1600 L=5.3 N=1.5\% C=1E-4 Fe=1.4E-3 D4 WN-NODIEL 14-18 AFTER JOB NO.311
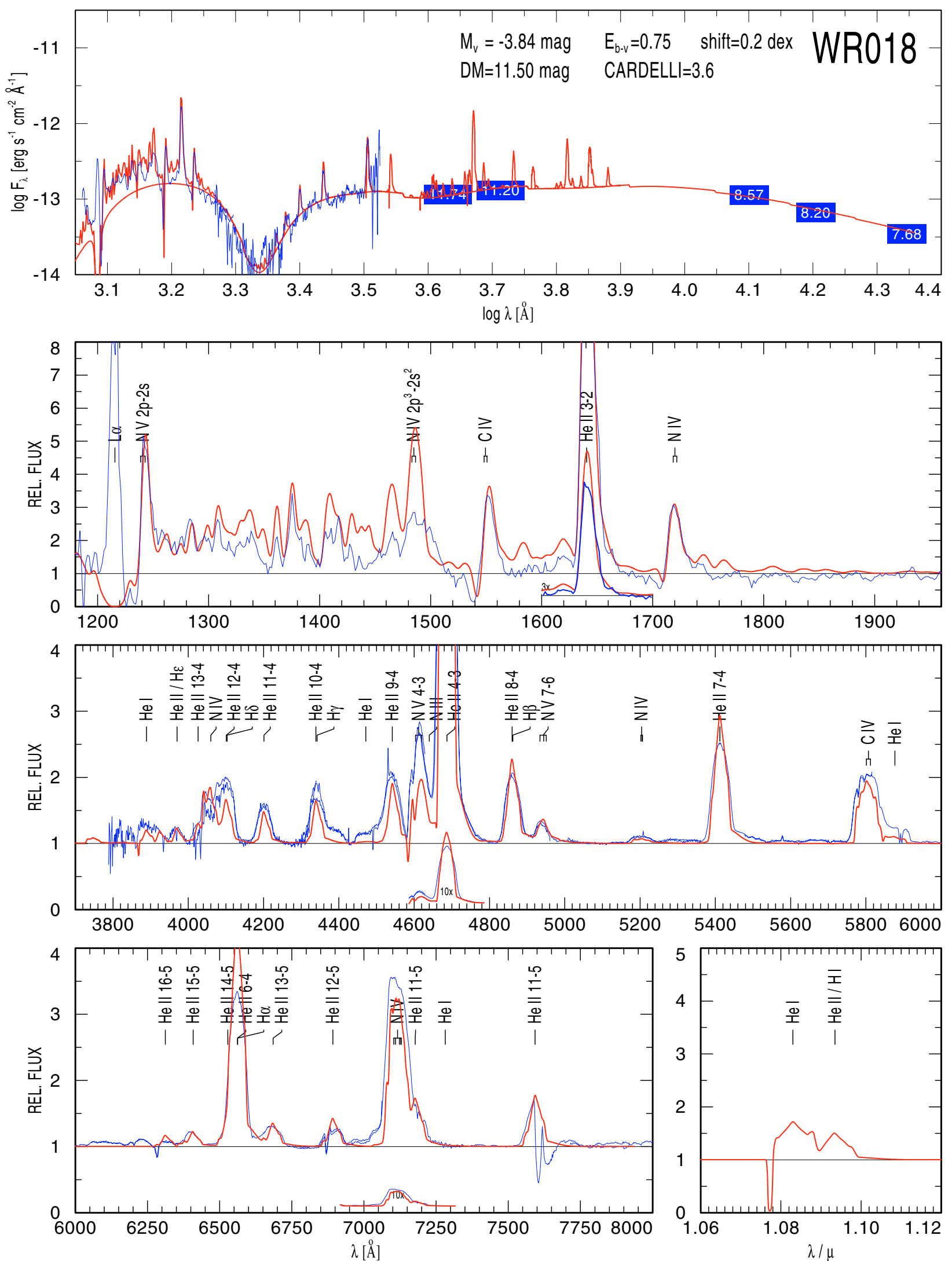

Fig. 20. Model: WNE 14-18, $T_{*}=112 \mathrm{kK}, \log \left(R_{\mathrm{t}} / R_{\odot}\right)=0.3$. 
W.-R. Hamann et al.: The Galactic WN stars, Online Material p 12

MODEL START 04/04/03 12:29:14 63096/0.9D/1600 L=5.3 N=1.5\% C=1E-4 Fe=1.4E-3 D4 WN-NODIEL 09-12 AFTER JOB NO.946
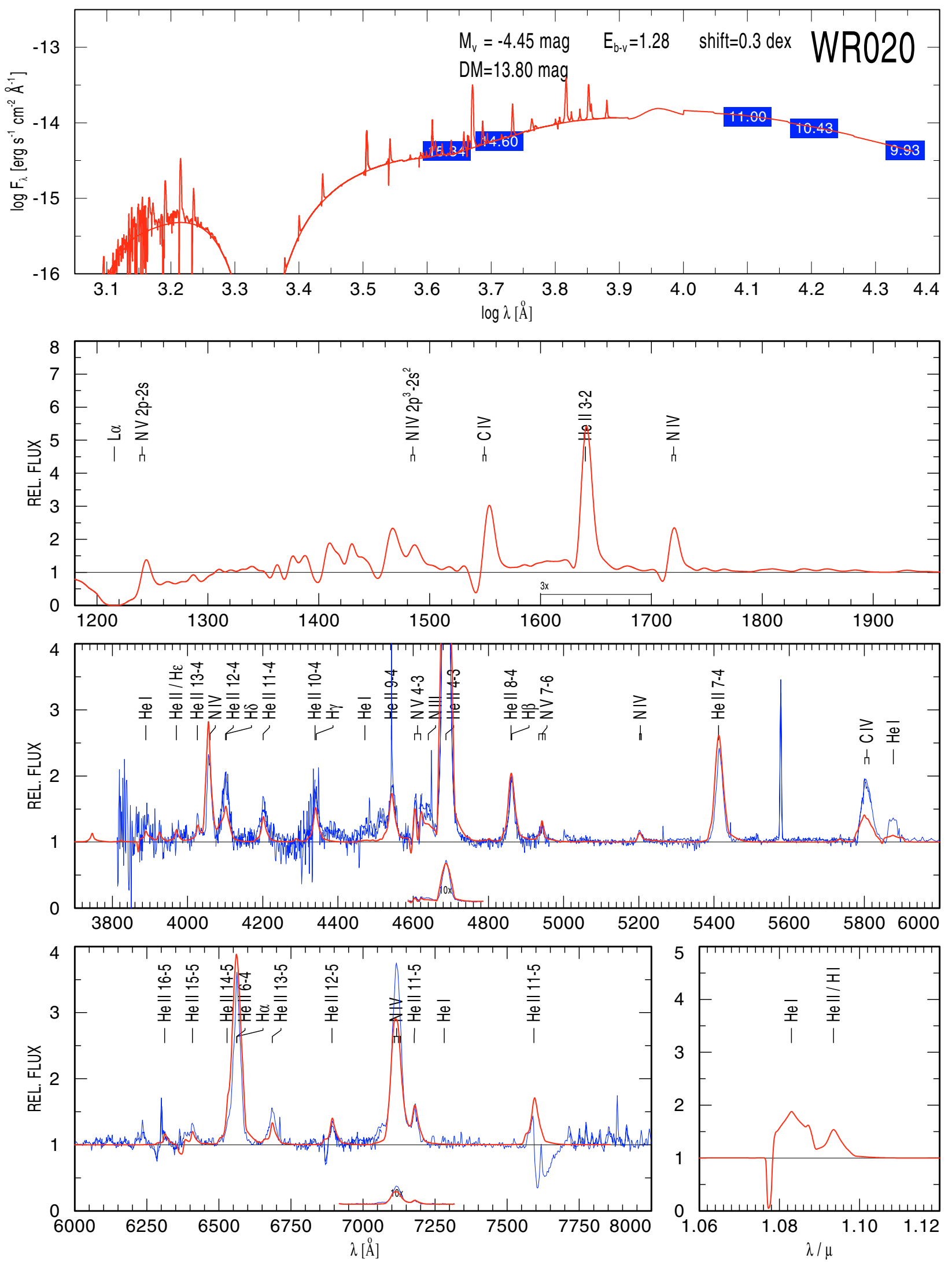

Fig. 21. Model: WNE 09-12, $T_{*}=63 \mathrm{kK}, \log \left(R_{\mathrm{t}} / R_{\odot}\right)=0.9$. 
W.-R. Hamann et al.: The Galactic WN stars, Online Material p 13

MODEL START 06/08/05 17:21:29 44668/1.30D/1800 L5.3 H40 N1.5\% C1E-4 Fe0.14\% D4 WNL-NODR 06-08 AFTER JOB NO.365
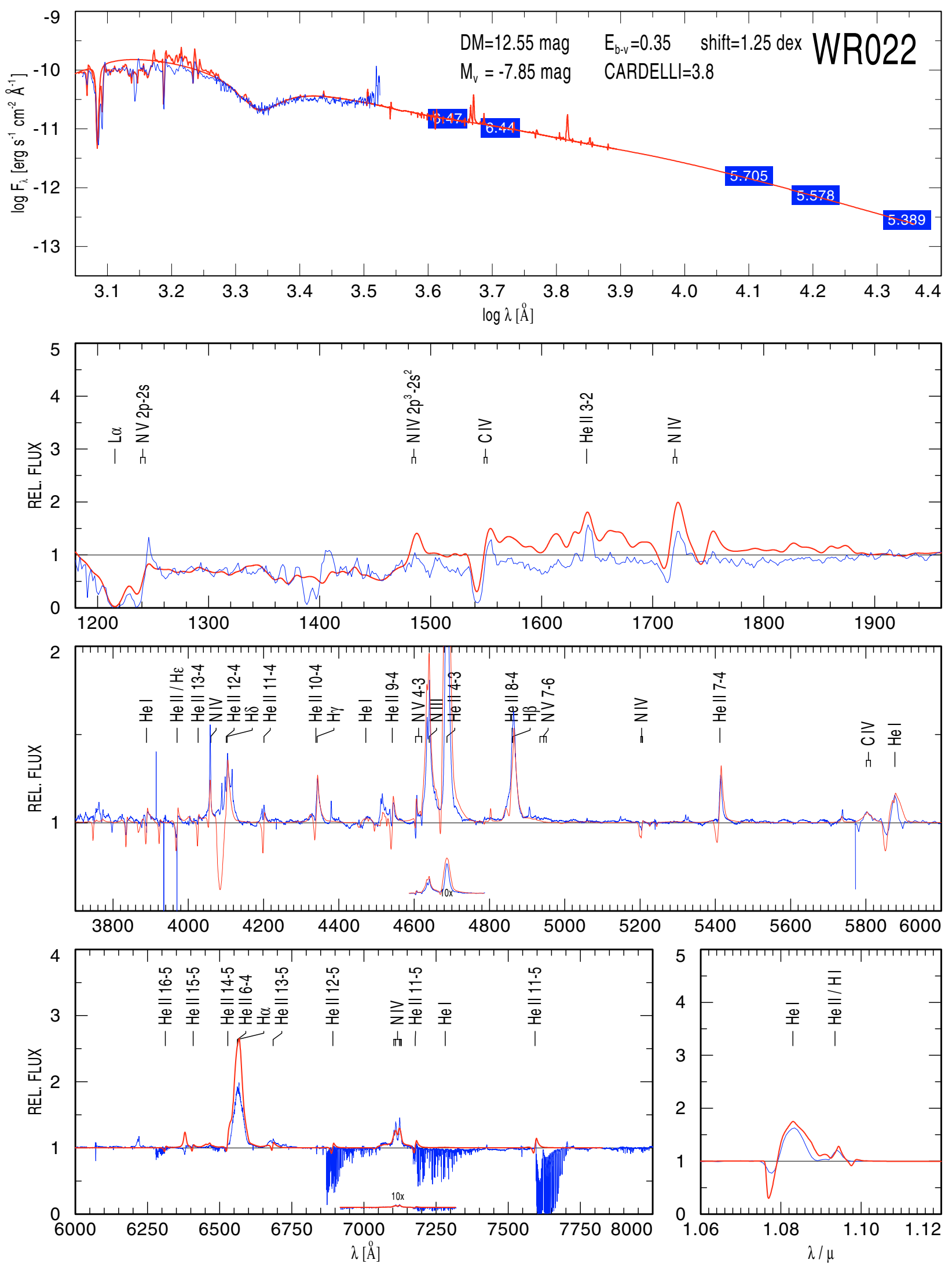

Fig. 22. Individual Model: 06-08, $T_{*}=45 \mathrm{kK}, \log \left(R_{\mathrm{t}} / R_{\odot}\right)=1.3$ with $v_{\infty}=1800 \mathrm{~km} \mathrm{~s}^{-1}$ and $X_{\mathrm{H}}=0.40$. 
W.-R. Hamann et al.: The Galactic WN stars, Online Material p 14

MODEL START 12/15/05 11:45:45 50119/1.35D/2000 L=5.3 H44 N1.5\% C1E-4 Fe.14\% D4 WNL-NODR 07-08sp AFTER JOB N0.237
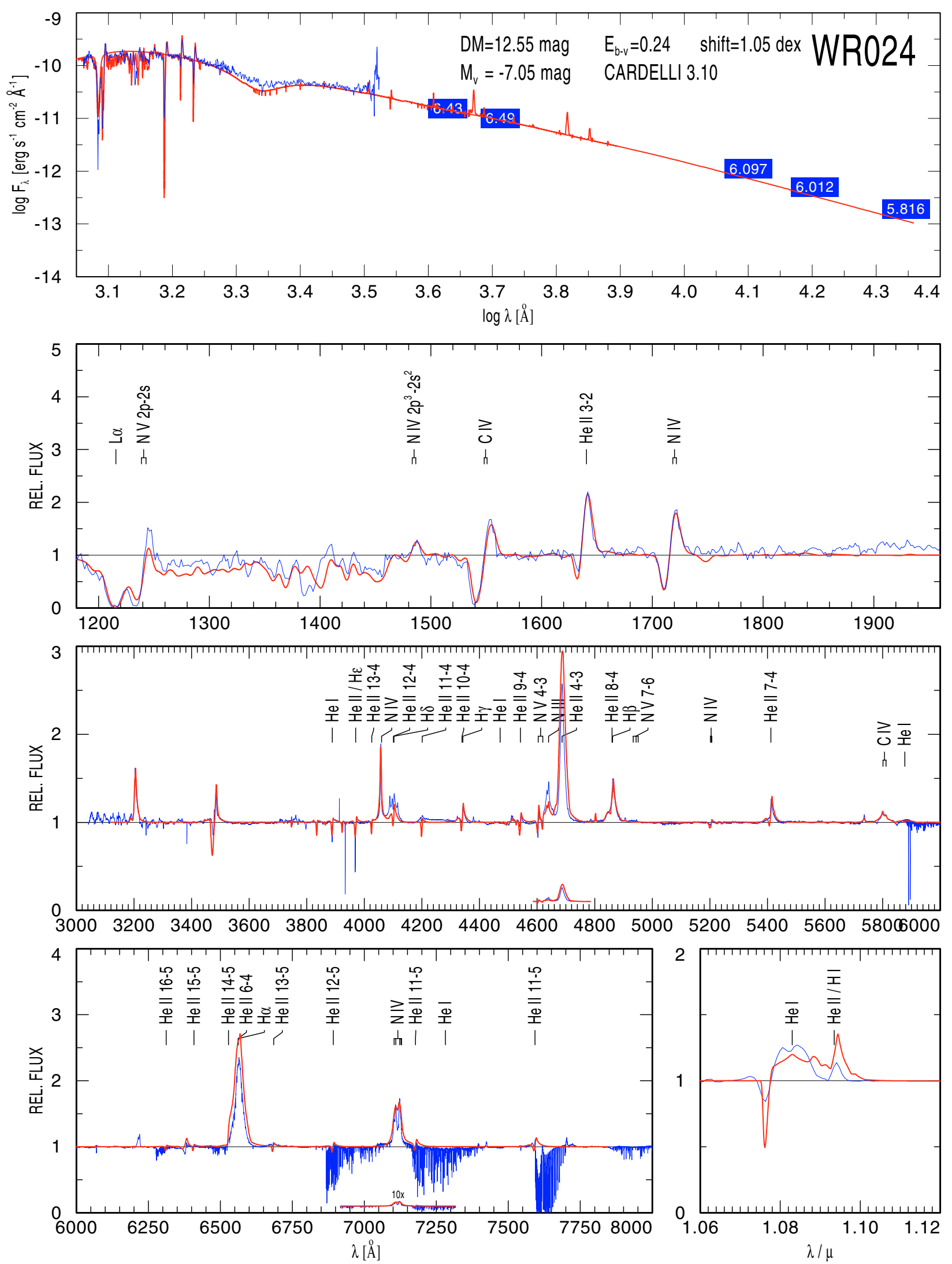

Fig. 23. Individual Model: 07-08, $T_{*}=50 \mathrm{kK}, \log \left(R_{\mathrm{t}} / R_{\odot}\right)=1.35$ with $v_{\infty}=2000 \mathrm{~km} \mathrm{~s}^{-1}$ and $X_{\mathrm{H}}=0.44$. 
W.-R. Hamann et al.: The Galactic WN stars, Online Material p 15

MODEL START 12/01/04 14:39:17 50119/1.5D/1800 L=5.3 H44 N1.5\% C1E-4 Fe0.14\% D4 WNL-NODR 07-06sp AFTER JOB N0.471
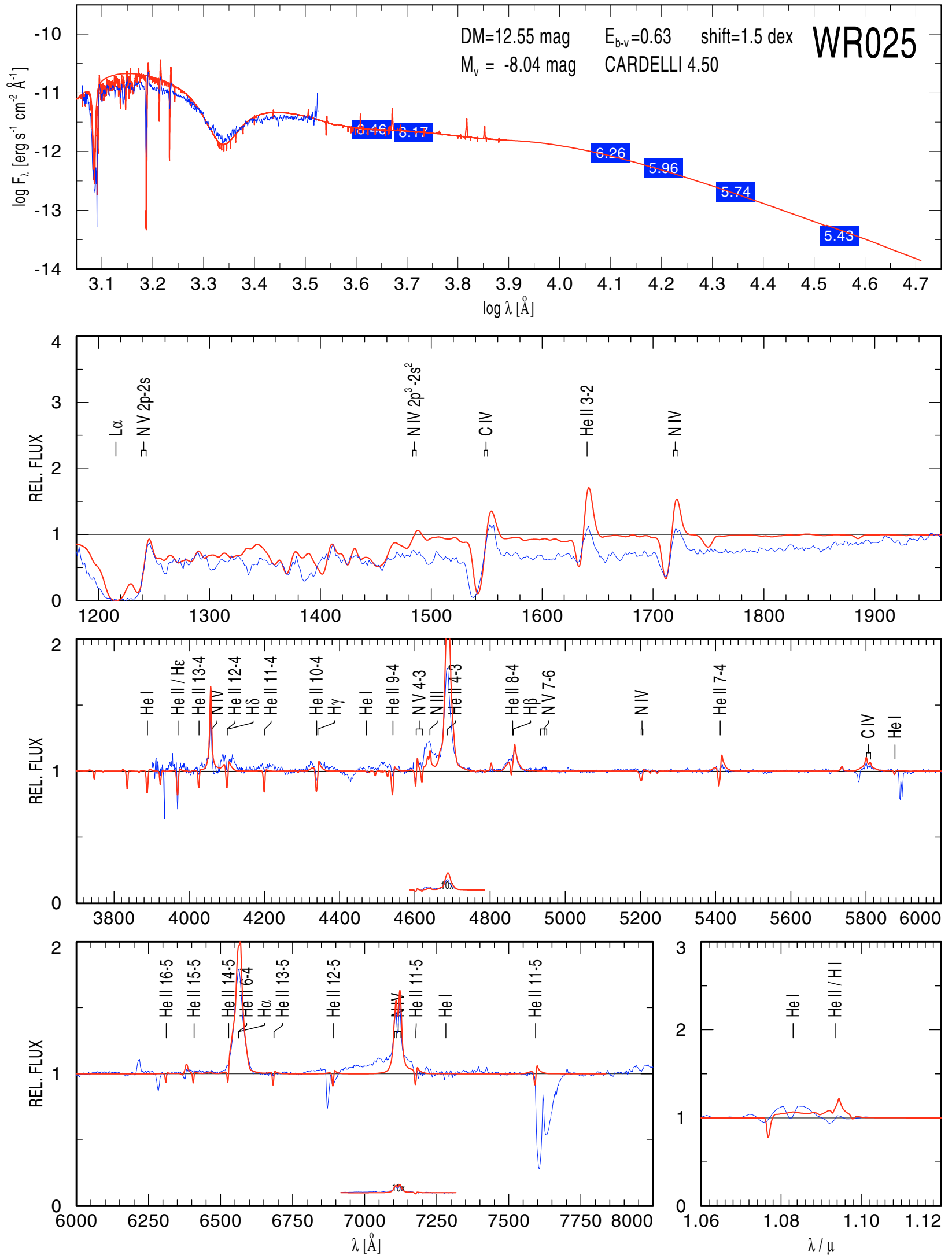

Fig. 24. Individual Model: 07-06, $T_{*}=50 \mathrm{kK}, \log \left(R_{\mathrm{t}} / R_{\odot}\right)=1.5$ with $v_{\infty}=1800 \mathrm{~km} \mathrm{~s}^{-1}$ and $X_{\mathrm{H}}=0.44$. 
W.-R. Hamann et al.: The Galactic WN stars, Online Material p 16

MODEL START 10/07/03 17:30:20 50119/1.2D/1000 L=5.3 H20 N1.5\% C1E-4 Fe0.14\% D4 WNL-NODR 7-09 AFTER JOB NO.109
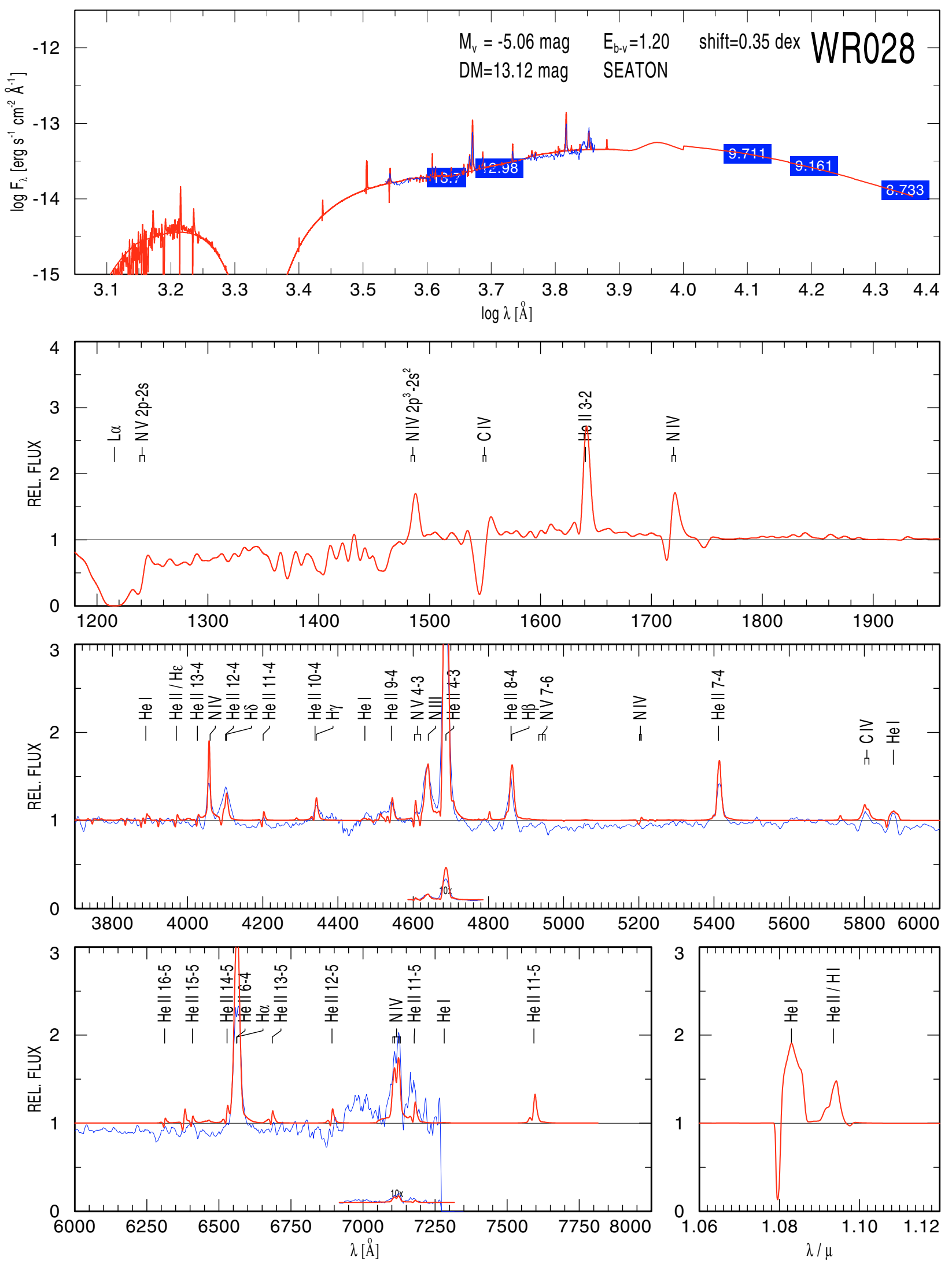

Fig. 25. Model: WNL 07-09, $T_{*}=50 \mathrm{kK}, \log \left(R_{\mathrm{t}} / R_{\odot}\right)=1.2$. 
W.-R. Hamann et al.: The Galactic WN stars, Online Material p 17

MODEL START 04/03/03 22:20:39 63096/0.8D/1600 L=5.3 N=1.5\% C=1E-4 Fe=1.4E-3 D4 WN-NODIEL 9-13 AFTER JOB N0.284
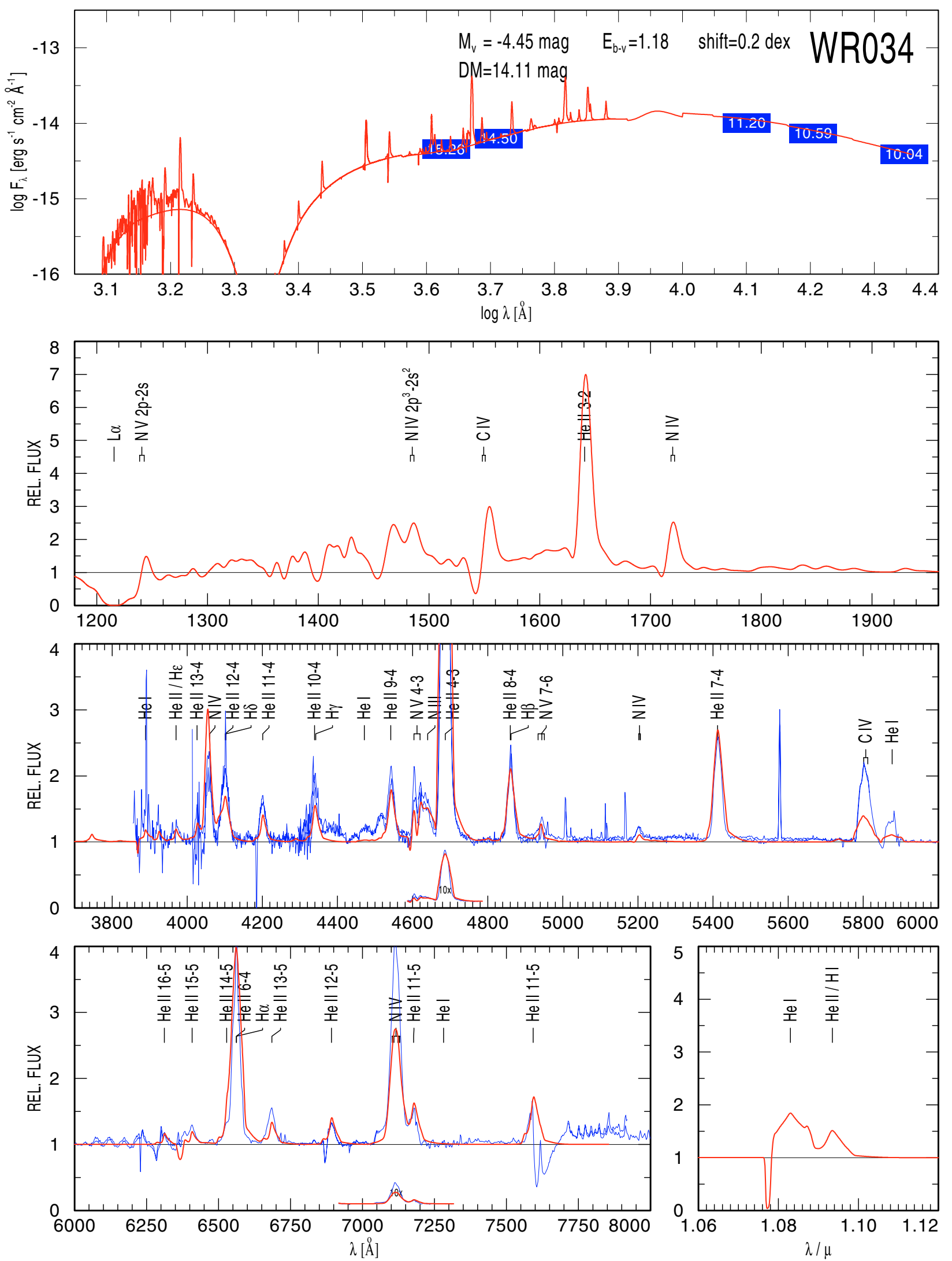

Fig. 26. Model: WNE 09-13, $T_{*}=63 \mathrm{kK}, \log \left(R_{\mathrm{t}} / R_{\odot}\right)=0.8$. 
W.-R. Hamann et al.: The Galactic WN stars, Online Material p 18

MODEL START 10/21/03 16:29:44 56234/0.90D/1000 L=5.3 H20 N1.5\% C1E-4 Fe0.14\% D4 WNL-NODR 08-12 AFTER JOB NO.587
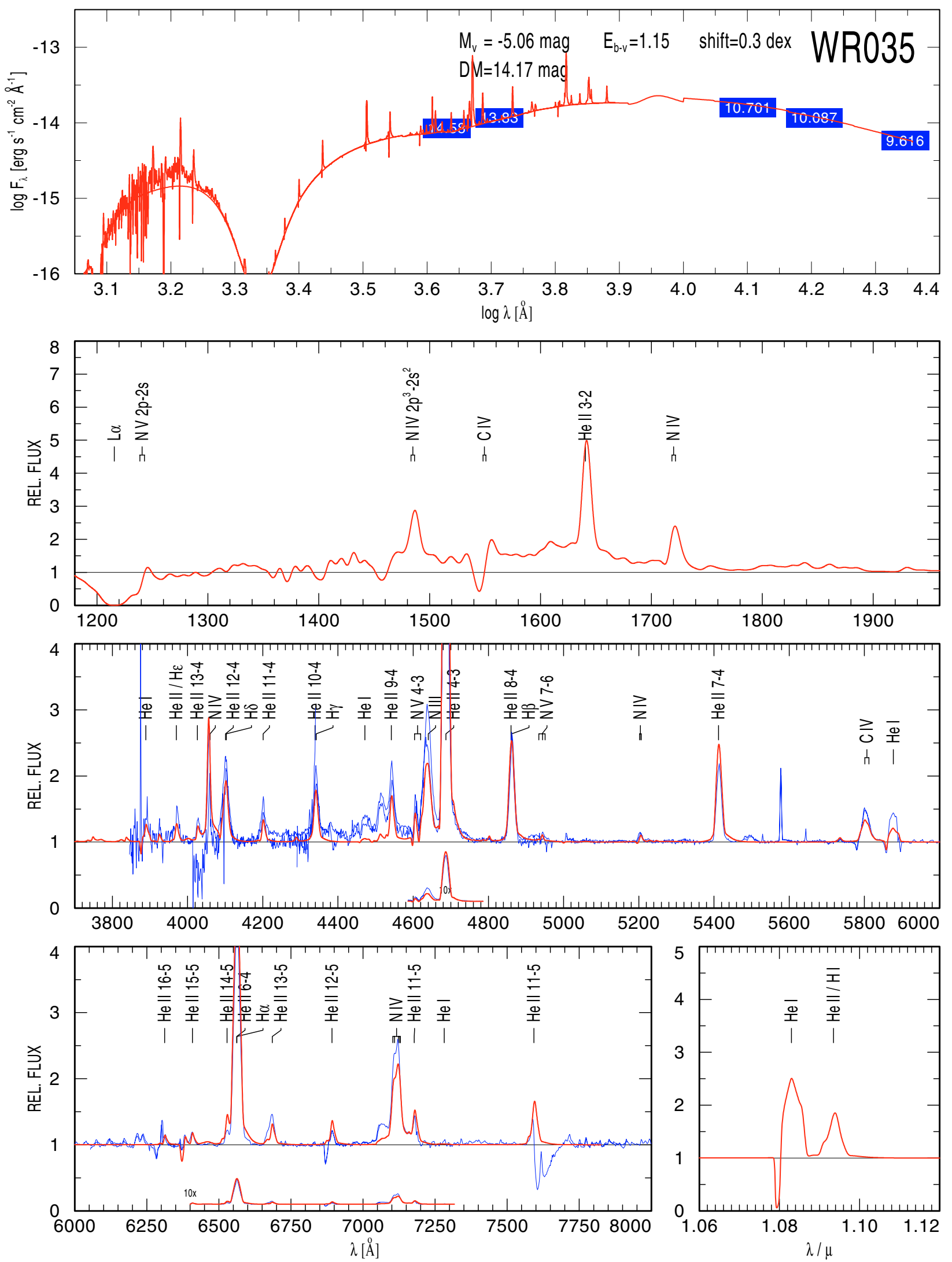

Fig. 27. Model: WNL 08-12, $T_{*}=56 \mathrm{kK}, \log \left(R_{\mathrm{t}} / R_{\odot}\right)=0.9$. 
W.-R. Hamann et al.: The Galactic WN stars, Online Material p 19

MODEL START 03/08/03 04:45:18 89125/0.2D/1600 L=5.3 N=1.5\% C=1E-4 Fe=1.4E-3 D4 WN-NODIEL 12-19 AFTER JOB NO.831
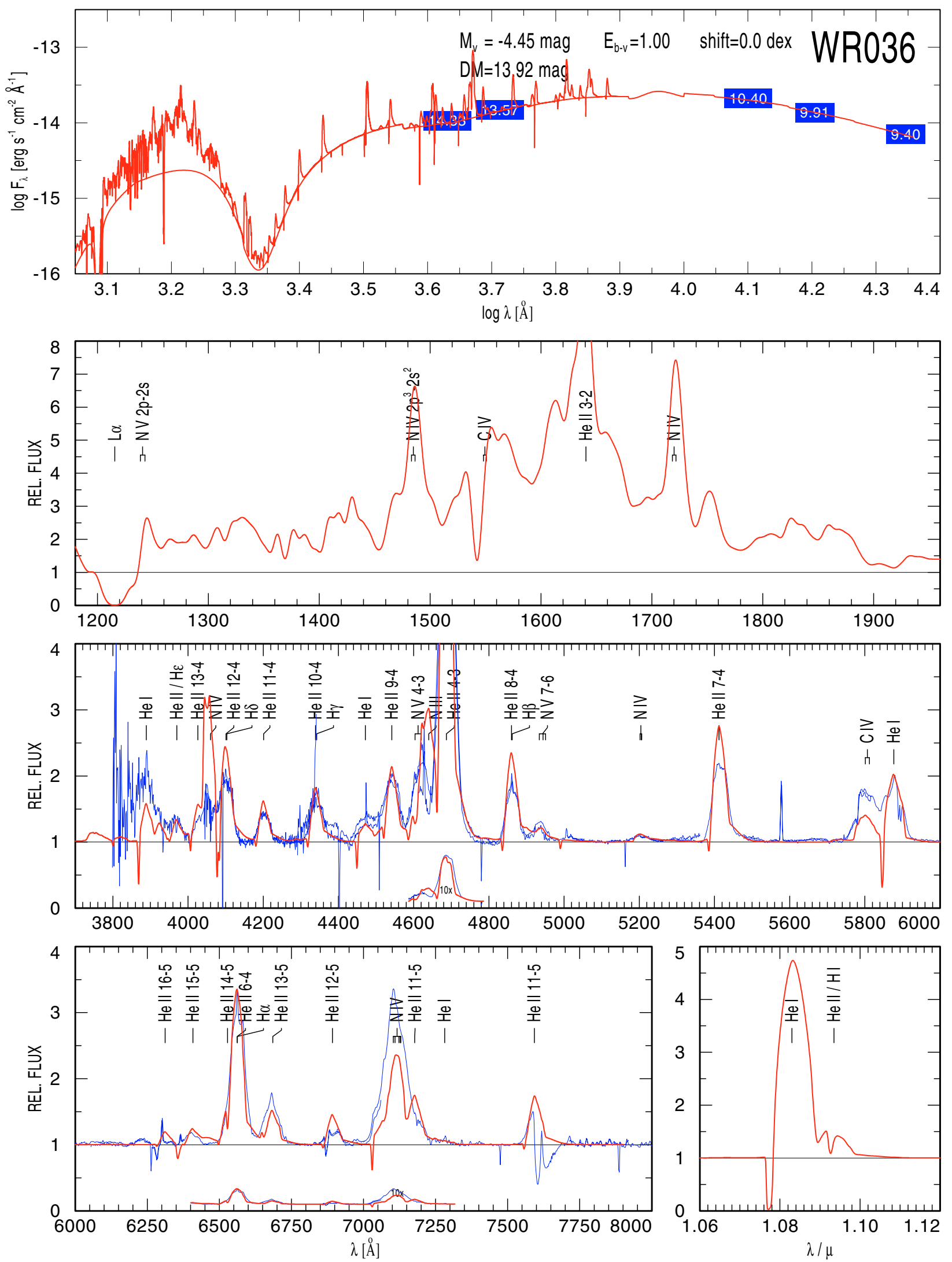

Fig. 28. Model: WNE 12-19, $T_{*}=89 \mathrm{kK}, \log \left(R_{\mathrm{t}} / R_{\odot}\right)=0.2$. 
W.-R. Hamann et al.: The Galactic WN stars, Online Material p 20

MODEL START 02/11/03 16:59:55 100000/0.4D/1600 L=5.3 N=1.5\% C=1E-4 Fe=1.4E-3 D4 WN-NODIEL 13-17 AFTER JOB NO.387
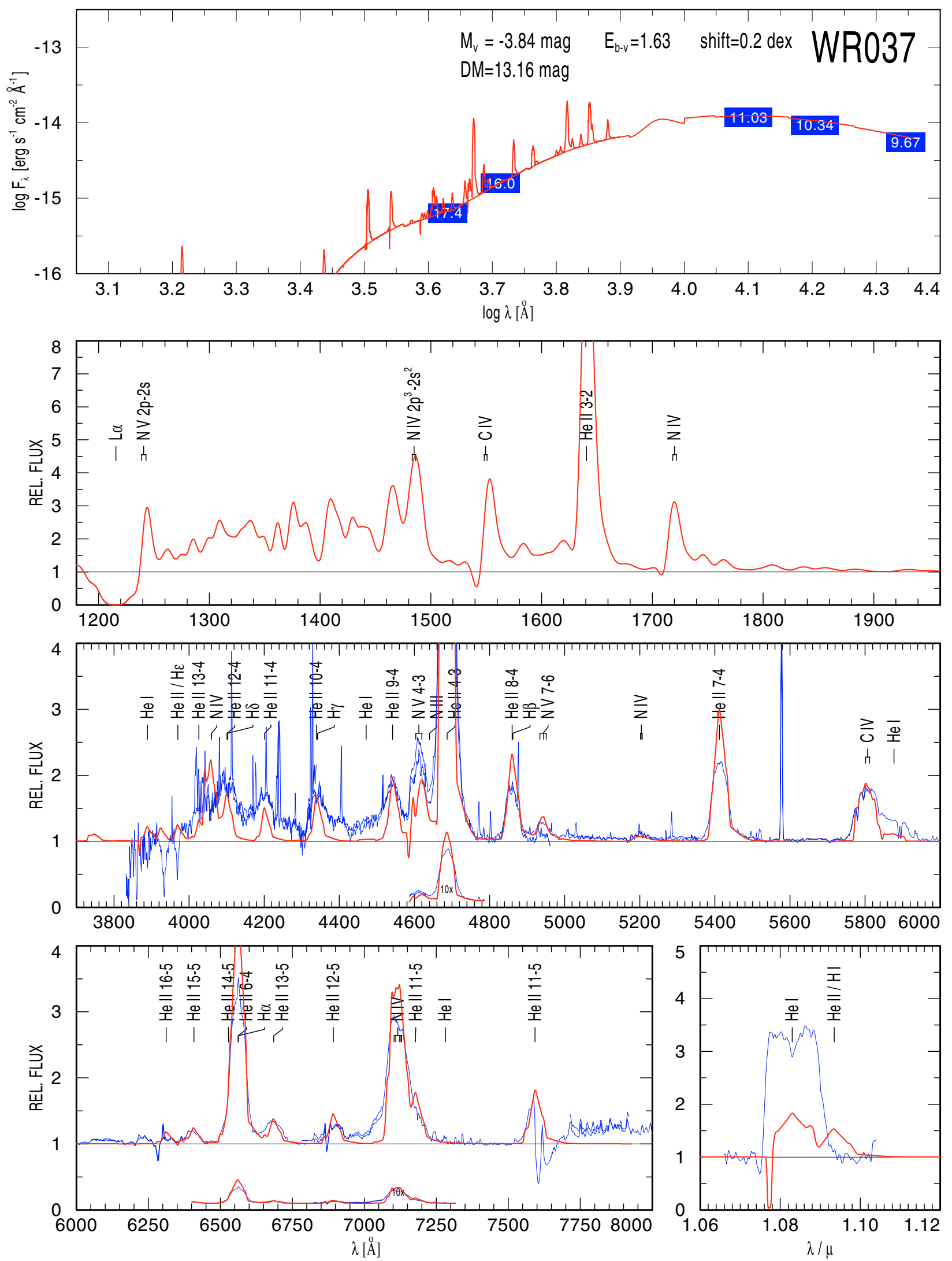

Fig. 29. Model: WNE 13-17, $T_{*}=100 \mathrm{kK}, \log \left(R_{\mathrm{t}} / R_{\odot}\right)=0.4$. 
W.-R. Hamann et al.: The Galactic WN stars, Online Material p 21

MODEL START 10/01/03 16:06:27 44668/0.70D/1000 L=5.3 H20 N1.5\% C1E-4 Fe0.14\% D4 WNL-NODR 06-14 AFTER JOB NO.514
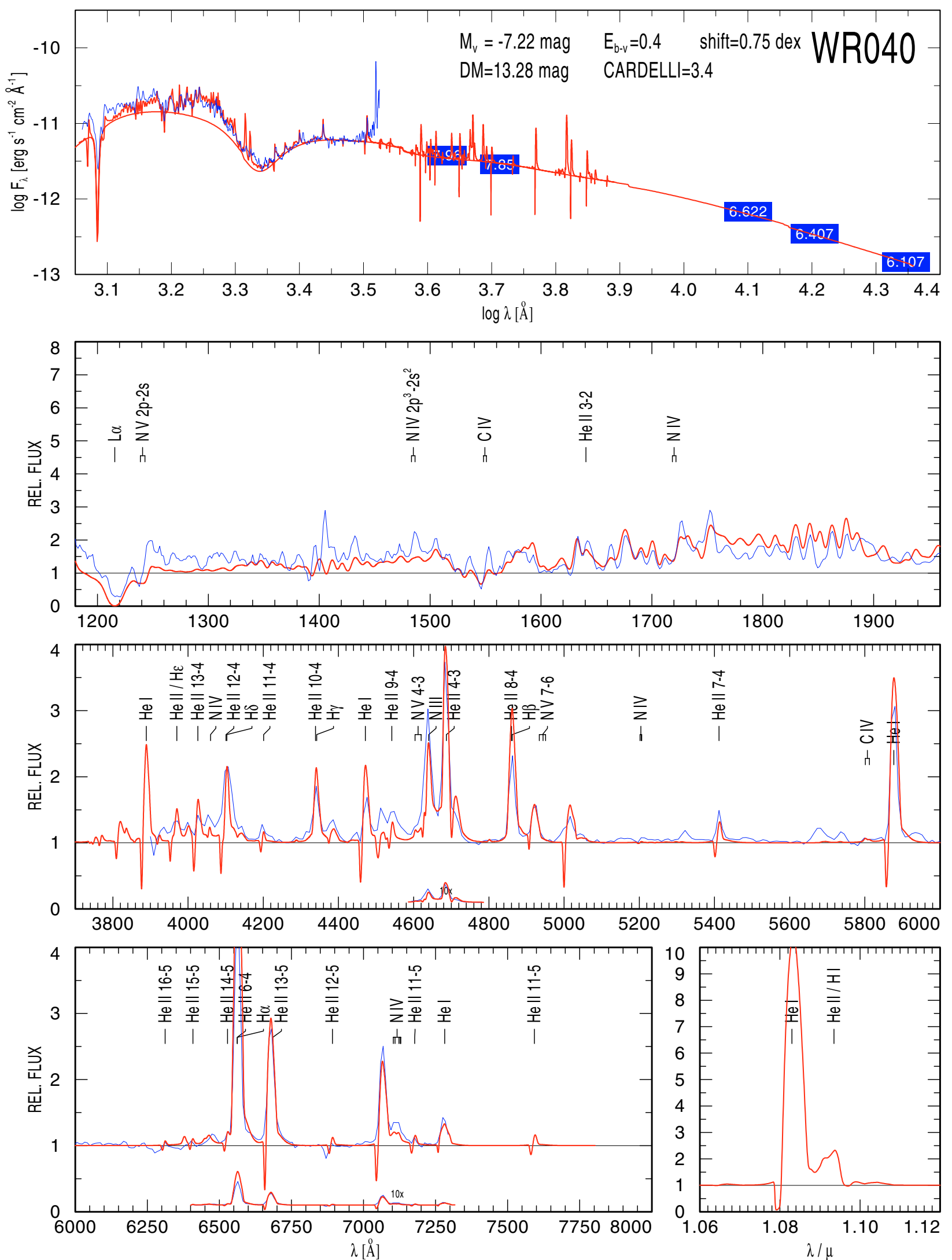

Fig. 30. Model: WNL 06-14, $T_{*}=45 \mathrm{kK}, \log \left(R_{\mathrm{t}} / R_{\odot}\right)=0.7$. 
W.-R. Hamann et al.: The Galactic WN stars, Online Material p 22

MODEL START 04/14/03 13:33:11 79433/0.8D/1600 L=5.3 N=1.5\% C=1E-4 Fe=1.4E-3 D4 WN-NODIEL 11-13 AFTER JOB NO.764
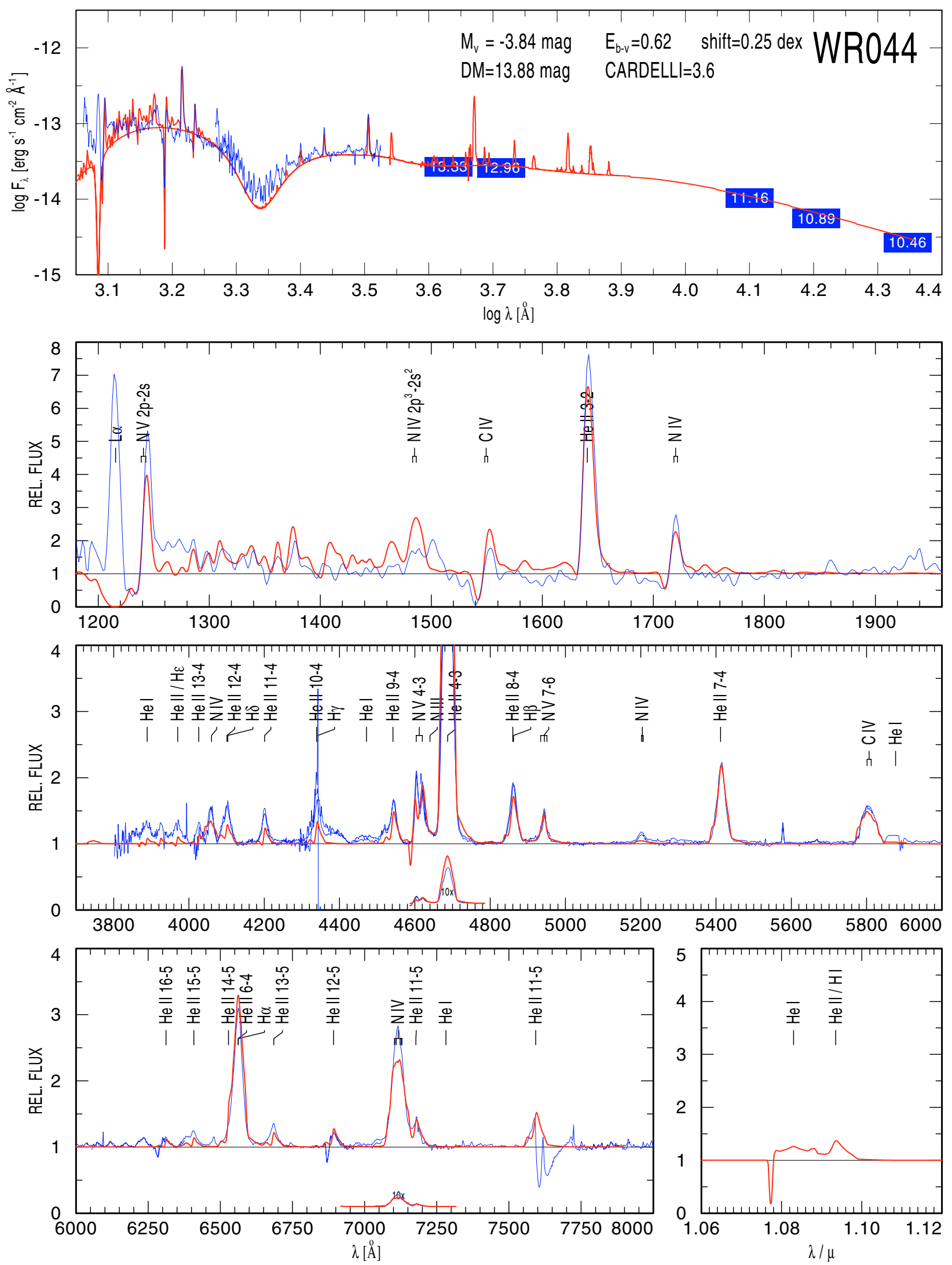

Fig. 31. Model: WNE $11-13, T_{*}=79 \mathrm{kK}, \log \left(R_{\mathrm{t}} / R_{\odot}\right)=0.8$. 
W.-R. Hamann et al.: The Galactic WN stars, Online Material p 23

MODEL START 10/13/05 18:09:59 112202/0.8D/2450 L=5.3 N=1.5\% C=1E-4 Fe=1.4E-3 D4 WN-NODR (14-13) AFTER JOB N0.406
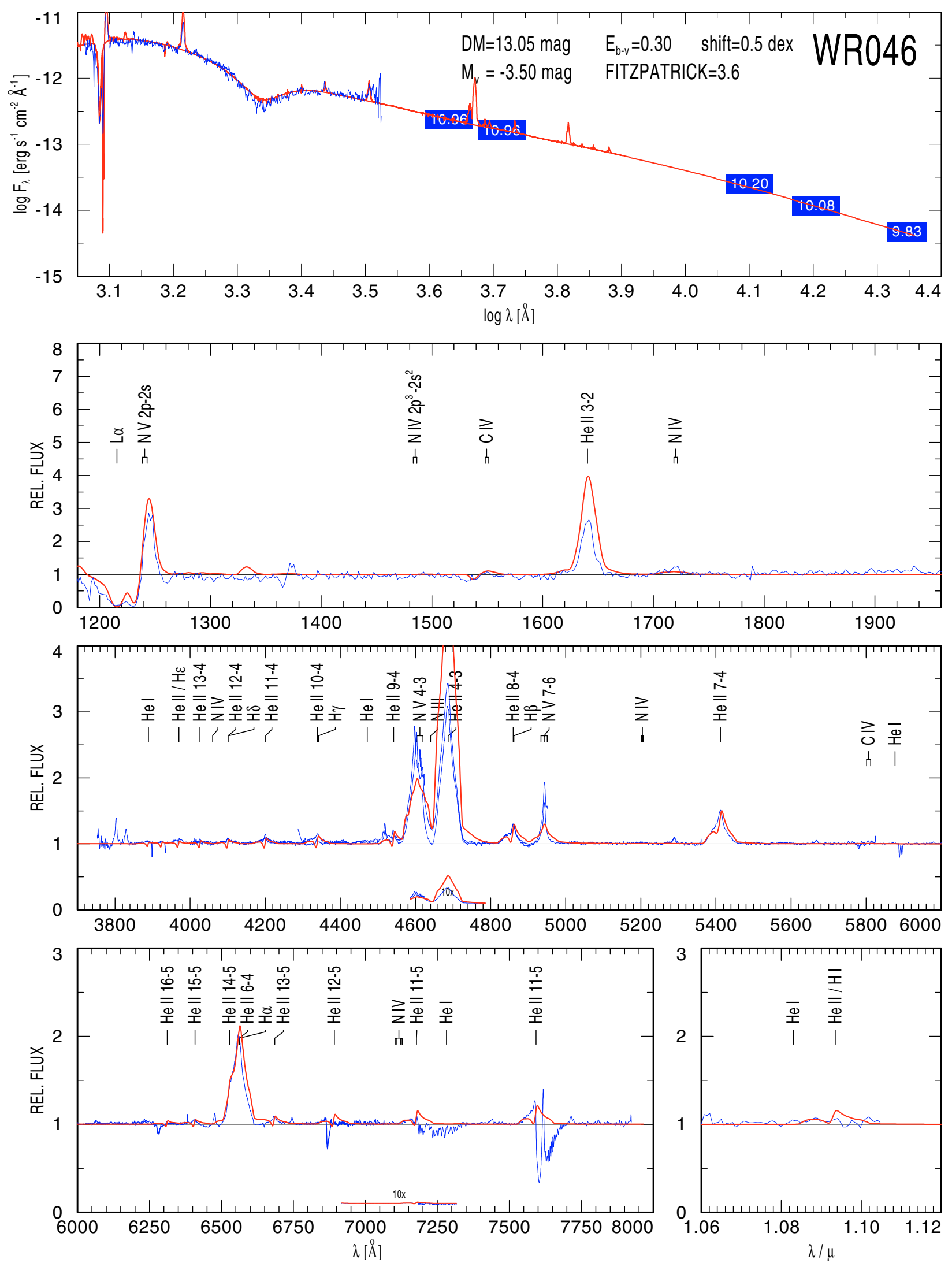

Fig. 32. Individual Model: WNE 14-13 $T_{*}=112 \mathrm{kK}, \log \left(R_{\mathrm{t}} / R_{\odot}\right)=0.8$ but $v_{\infty}=2450 \mathrm{~km} \mathrm{~s}^{-1}$. 
W.-R. Hamann et al.: The Galactic WN stars, Online Material p 24

MODEL START 10/21/03 16:22:34 56234/1.0D/1000 L=5.3 H20 N1.5\% C1E-4 Fe0.14\% D4 WNL-NODR 08-11 AFTER JOB NO.472
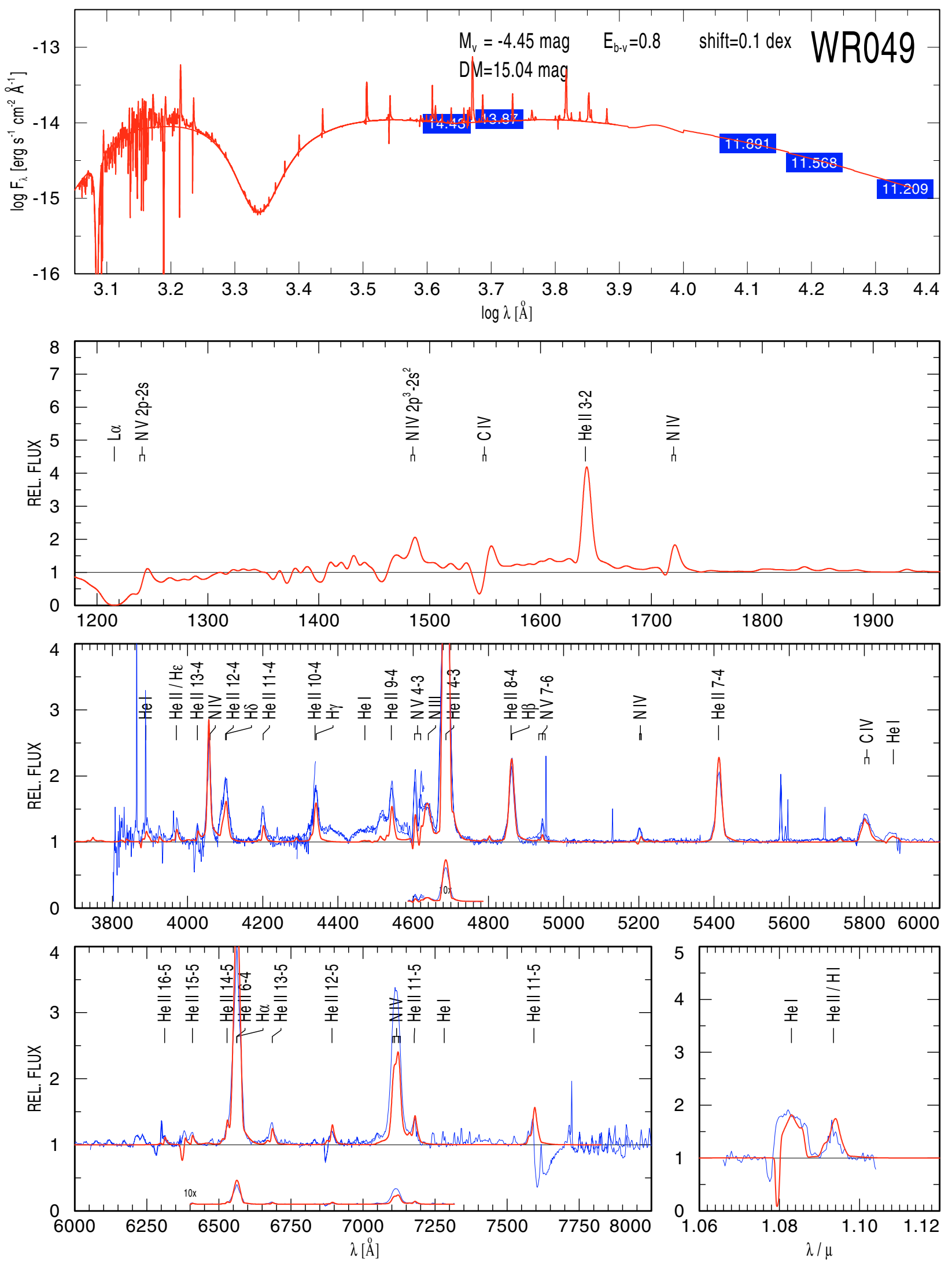

Fig. 33. Model: WNL 08-11, $T_{*}=56 \mathrm{kK}, \log \left(R_{\mathrm{t}} / R_{\odot}\right)=1.0$. 
W.-R. Hamann et al.: The Galactic WN stars, Online Material p 25

MODEL START 03/28/03 03:43:21 70795/0.9D/1600 L=5.3 N=1.5\% C=1E-4 Fe=1.4E-3 D4 WN-NODIEL 10-12 AFTER JOB NO.609
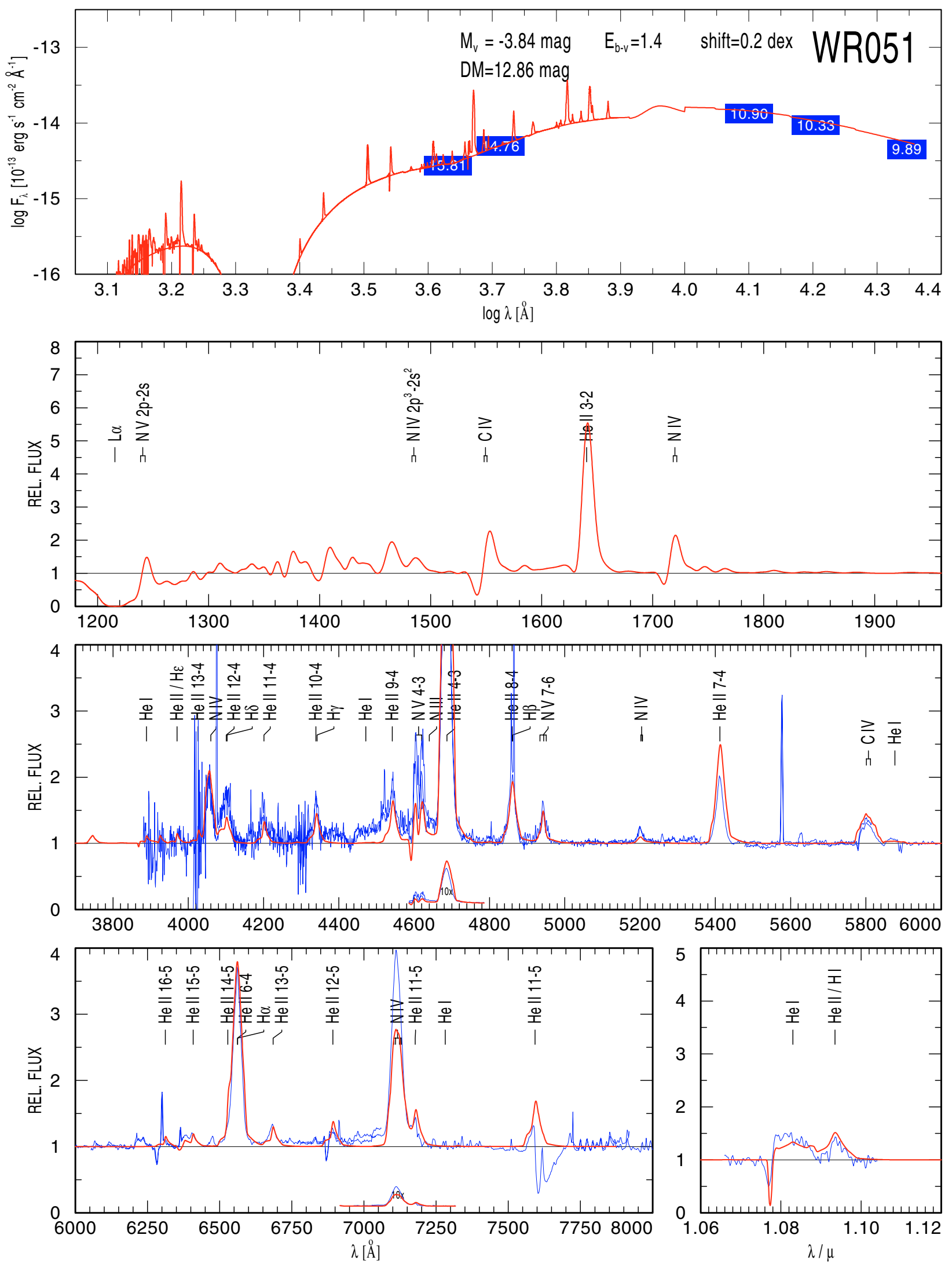

Fig. 34. Model: WNE $10-12, T_{*}=71 \mathrm{kK}, \log \left(R_{\mathrm{t}} / R_{\odot}\right)=0.9$. 
W.-R. Hamann et al.: The Galactic WN stars, Online Material p 26

MODEL START 04/04/03 12:29:14 63096/0.9D/1600 L=5.3 N=1.5\% C=1E-4 Fe=1.4E-3 D4 WN-NODIEL 09-12 AFTER JOB NO.946
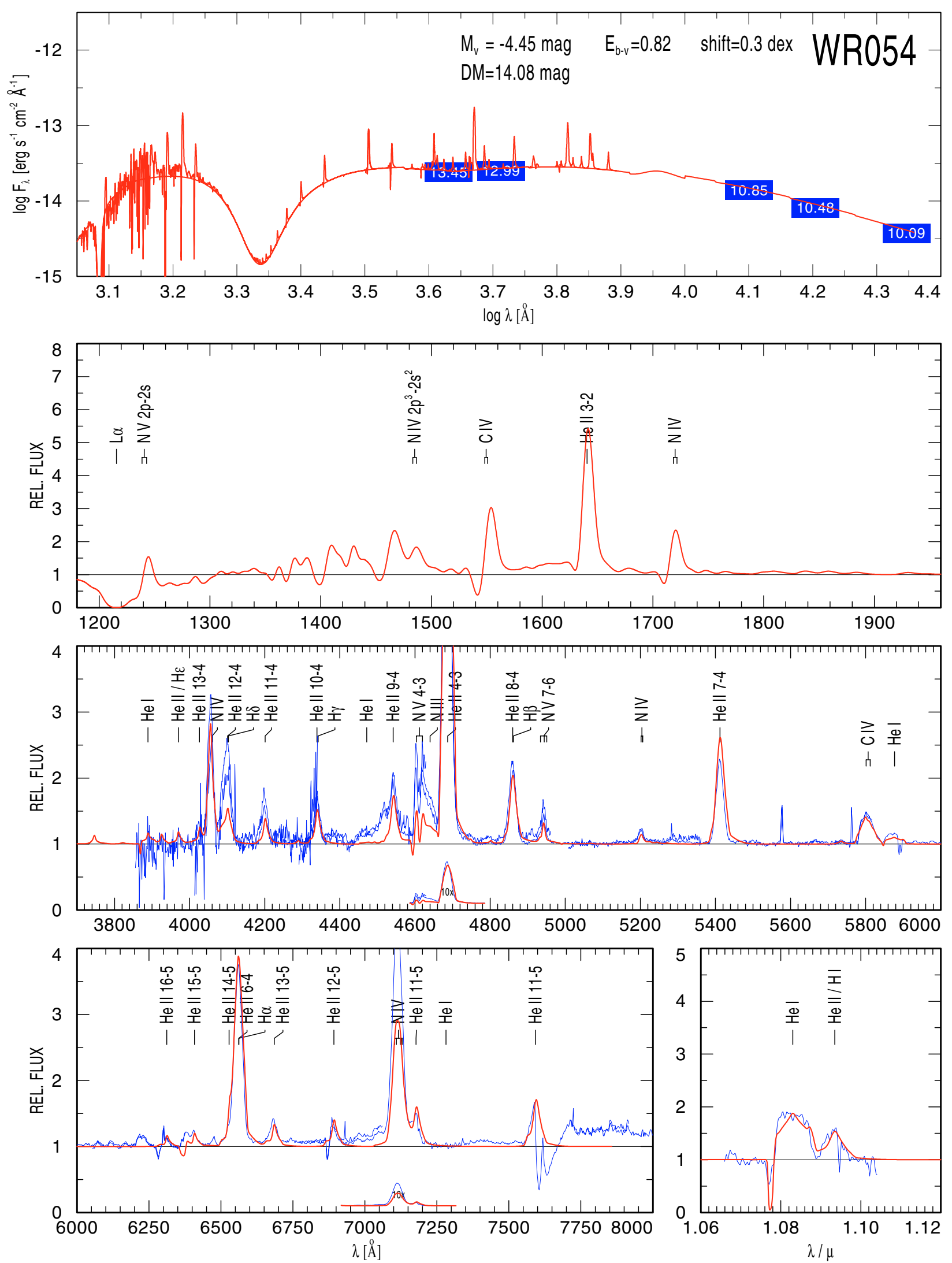

Fig. 35. Model: WNE 09-12, $T_{*}=63 \mathrm{kK}, \log \left(R_{\mathrm{t}} / R_{\odot}\right)=0.9$. 
W.-R. Hamann et al.: The Galactic WN stars, Online Material p 27

MODEL START 04/21/03 23:53:29 56234/0.8D/1600 L=5.3 N=1.5\% C=1E-4 Fe=1.4E-3 D4 WN-NODIEL 08-13 AFTER JOB NO.825
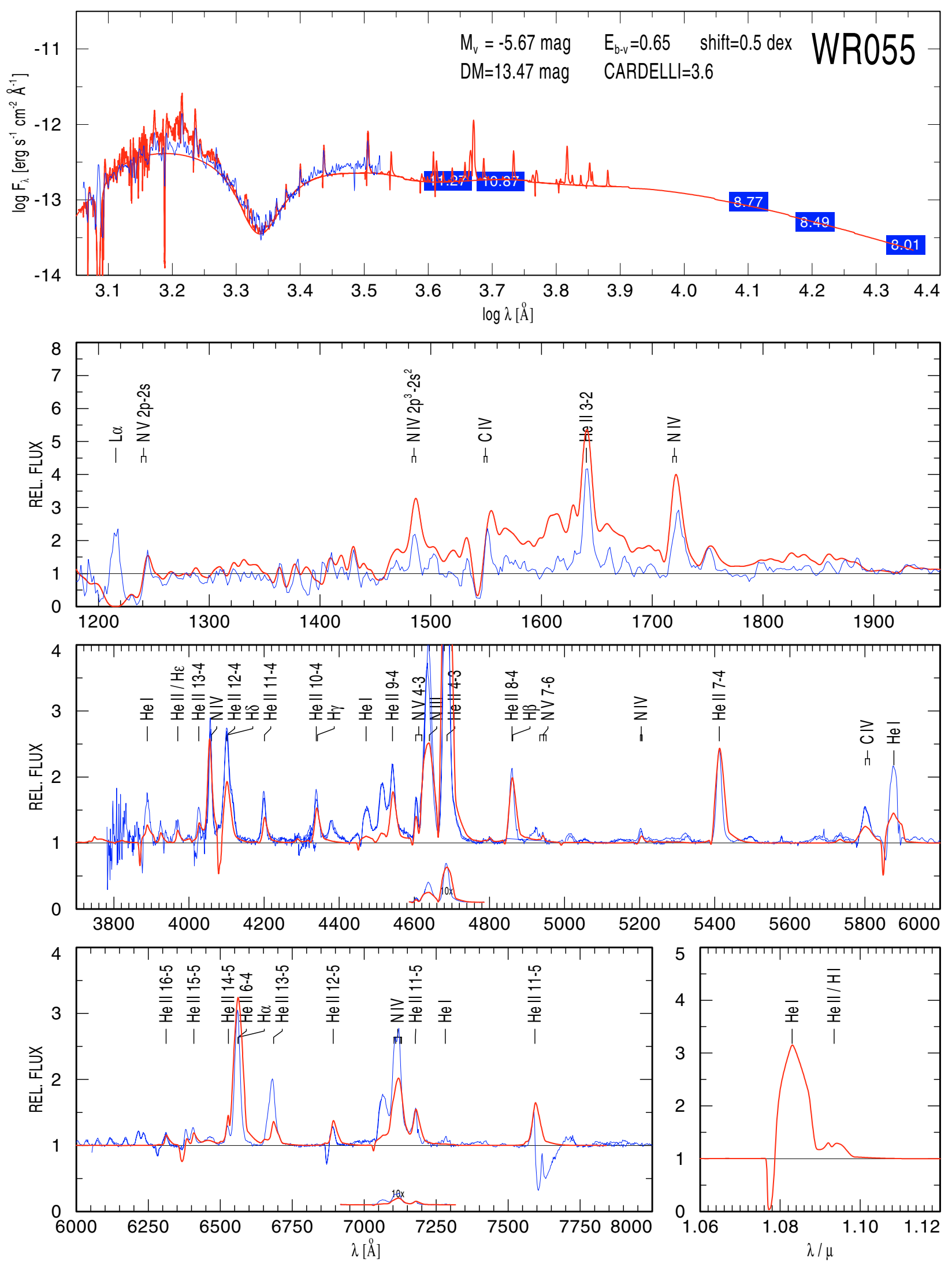

Fig. 36. Model: WNE 08-13, $T_{*}=56 \mathrm{kK}, \log \left(R_{\mathrm{t}} / R_{\odot}\right)=0.8$. 
W.-R. Hamann et al.: The Galactic WN stars, Online Material p 28

MODEL START 03/31/03 23:18:48 63096/0.7D/1600 L=5.3 N=1.5\% C=1E-4 Fe=1.4E-3 D4 WN-NODIEL 9-14 AFTER JOB NO.425
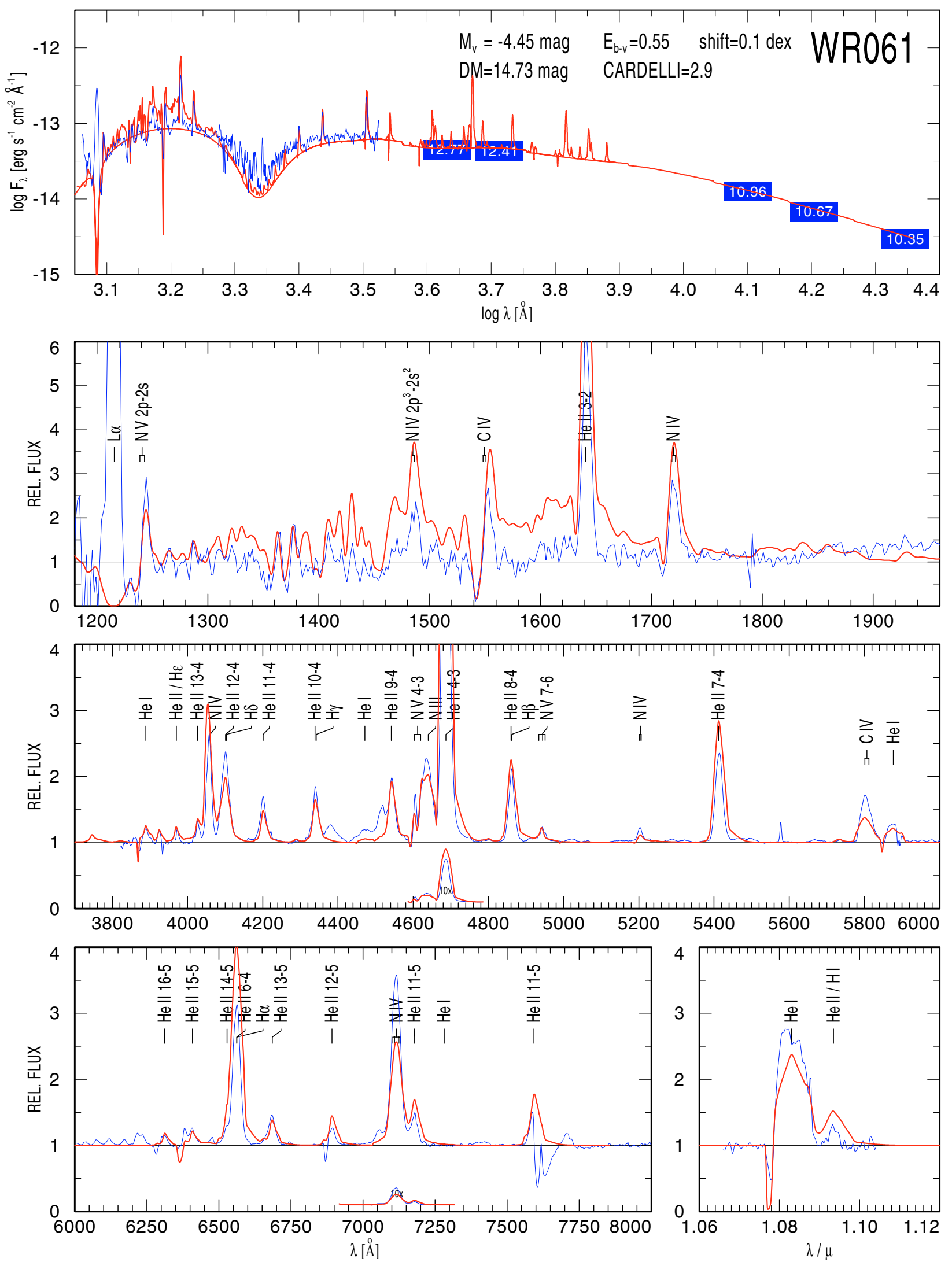

Fig. 37. Model: WNE 09-14, $T_{*}=63 \mathrm{kK}, \log \left(R_{\mathrm{t}} / R_{\odot}\right)=0.7$. 
W.-R. Hamann et al.: The Galactic WN stars, Online Material p 29

MODEL START 03/19/03 09:03:38 70795/0.4D/1600 L=5.3 N=1.5\% C=1E-4 Fe=1.4E-3 D4 WN-NODIEL 10-17 AFTER JOB NO.413
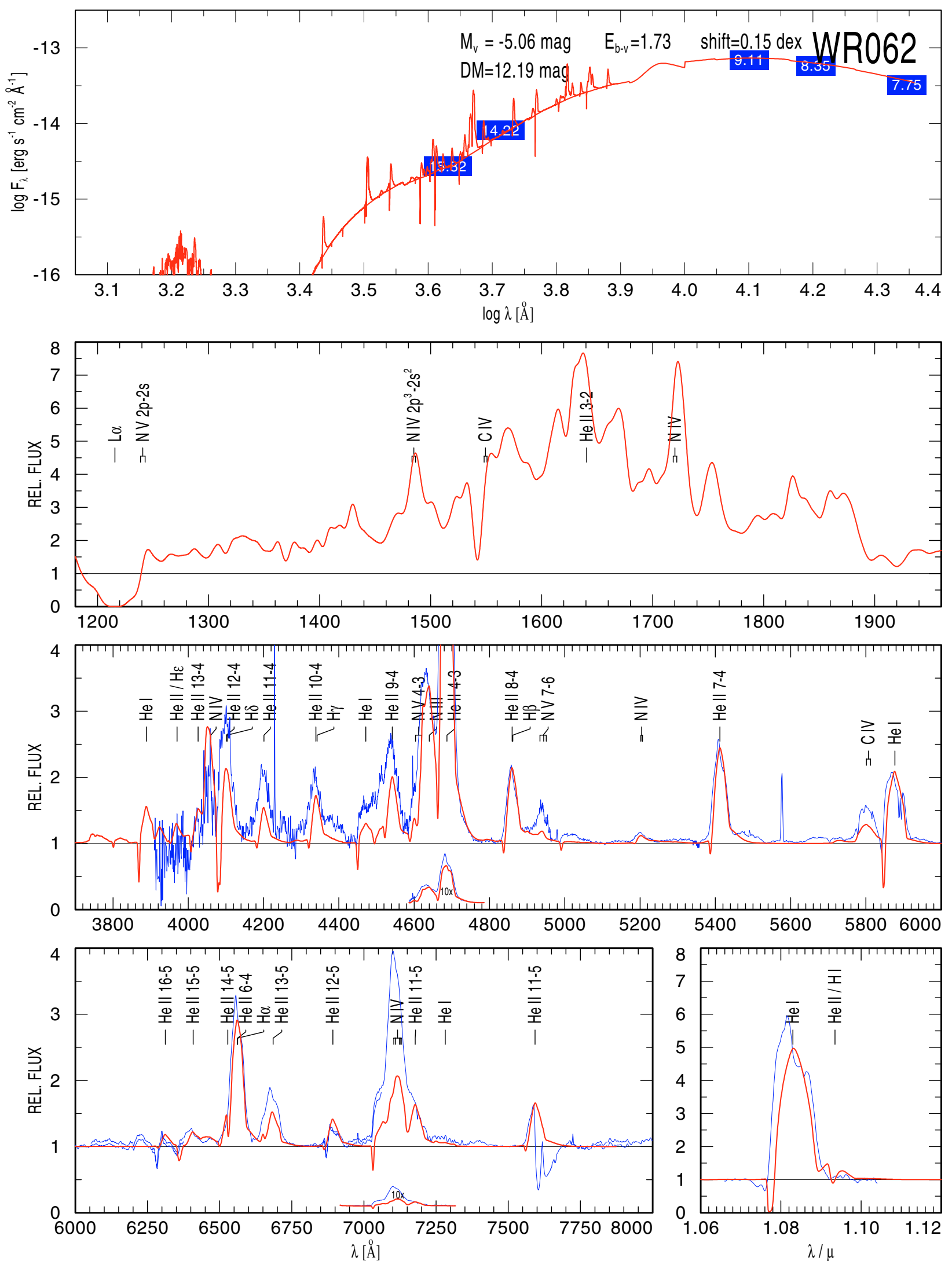

Fig. 38. Model: WNE 10-17, $T_{*}=71 \mathrm{kK}, \log \left(R_{\mathrm{t}} / R_{\odot}\right)=0.4$. 
W.-R. Hamann et al.: The Galactic WN stars, Online Material p 30

MODEL START 05/29/03 23:32:49 44668/1.1D/1600 L=5.3 N=1.5\% C=1E-4 Fe=1.4E-3 D4 WN-NODIEL 6-10 AFTER JOB N0.264
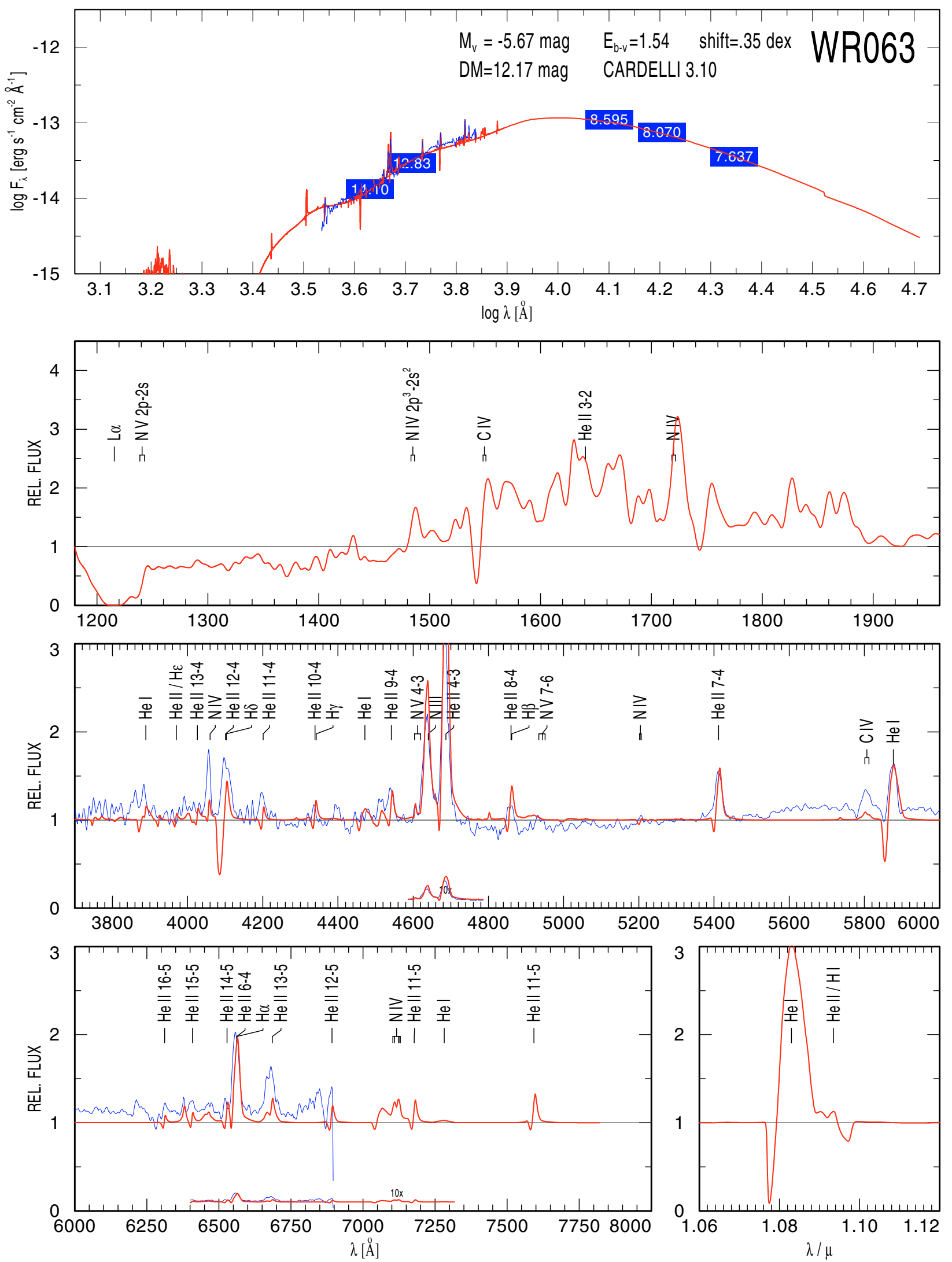

Fig. 39. Model: WNE 06-10, $T_{*}=45 \mathrm{kK}, \log \left(R_{\mathrm{t}} / R_{\odot}\right)=1.1$. 
W.-R. Hamann et al.: The Galactic WN stars, Online Material p 31

MODEL START 11/25/03 12:51:17 44668/0.9D/1000 L5.3 H20 N1.5\% C1E-4 Fe0.14\% D4 WNL-NODR 06-12 AFTER JOB N0.667
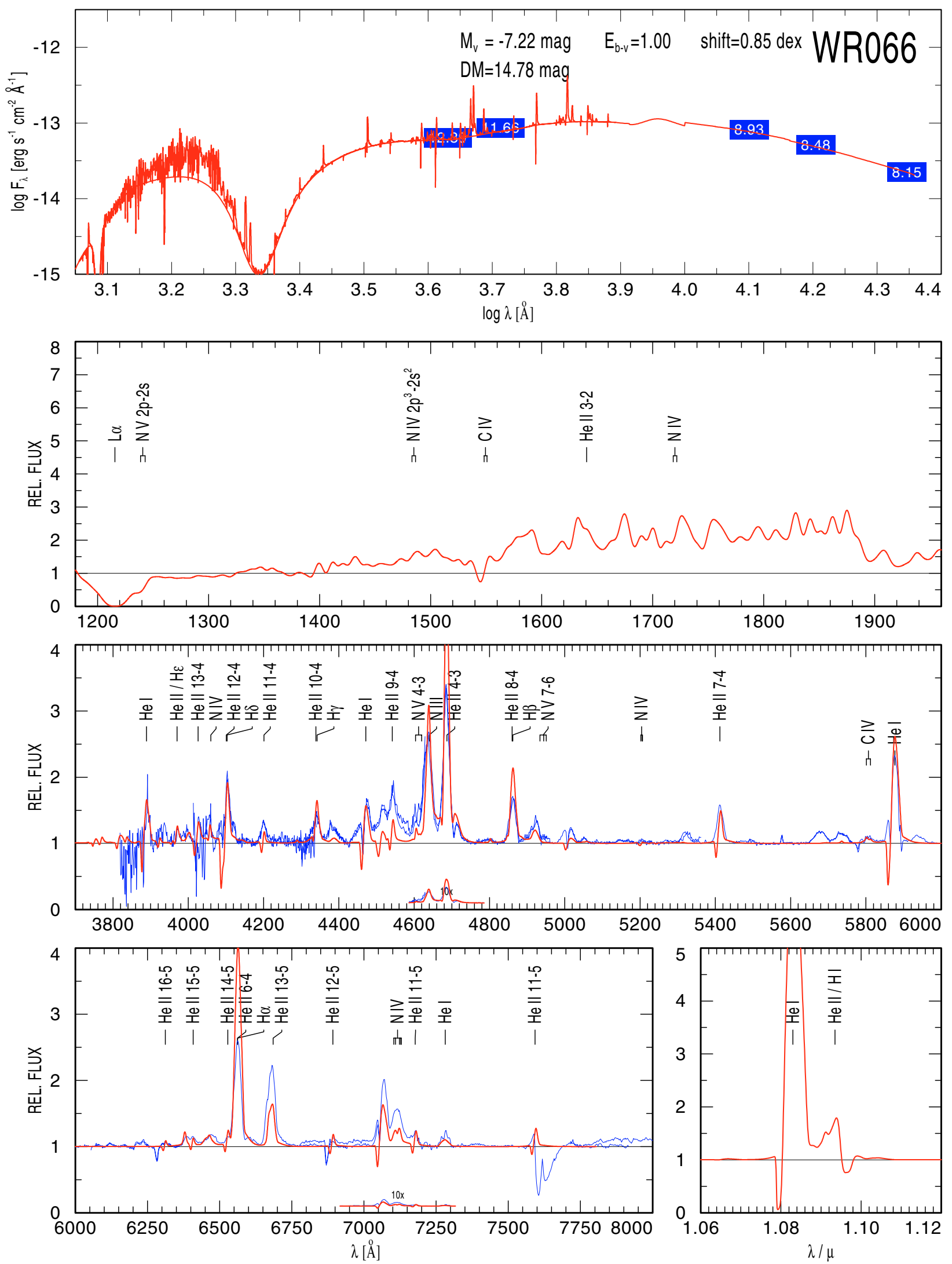

Fig. 40. Model: WNL 06-12, $T_{*}=45 \mathrm{kK}, \log \left(R_{\mathrm{t}} / R_{\odot}\right)=0.9$. 
W.-R. Hamann et al.: The Galactic WN stars, Online Material p 32

MODEL START 04/21/03 23:53:29 56234/0.8D/1600 L=5.3 N=1.5\% C=1E-4 Fe=1.4E-3 D4 WN-NODIEL 08-13 AFTER JOB NO.825
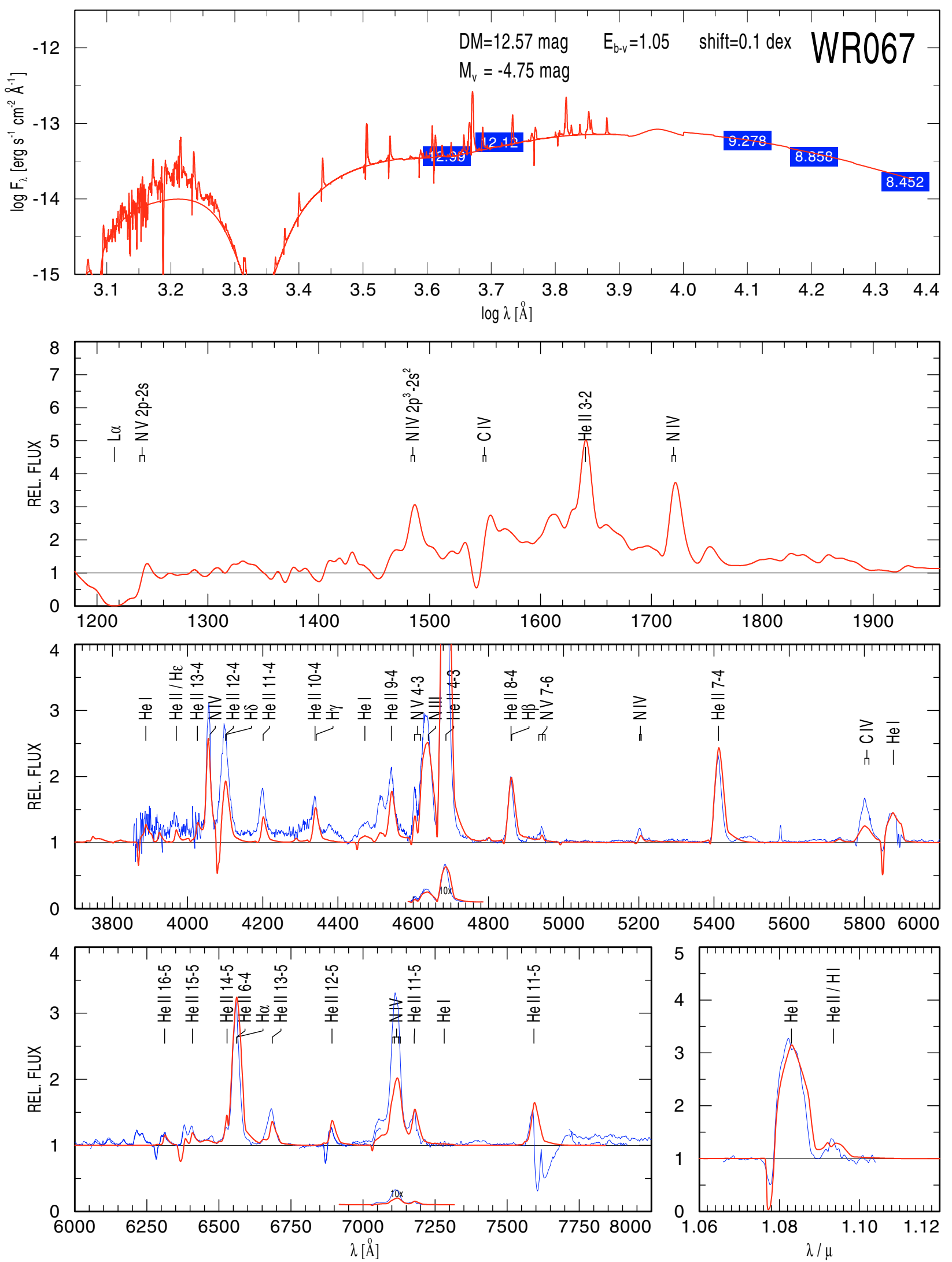

Fig. 41. Model: WNE 08-13, $T_{*}=56 \mathrm{kK}, \log \left(R_{\mathrm{t}} / R_{\odot}\right)=0.8$. 
W.-R. Hamann et al.: The Galactic WN stars, Online Material p 33

MODEL START 04/20/03 00:18:54 56234/0.90D/1600 L=5.3 N=1.5\% C=1E-4 Fe=1.4E-3 D4 WN-NODIEL 08-12 AFTER JOB N0.172
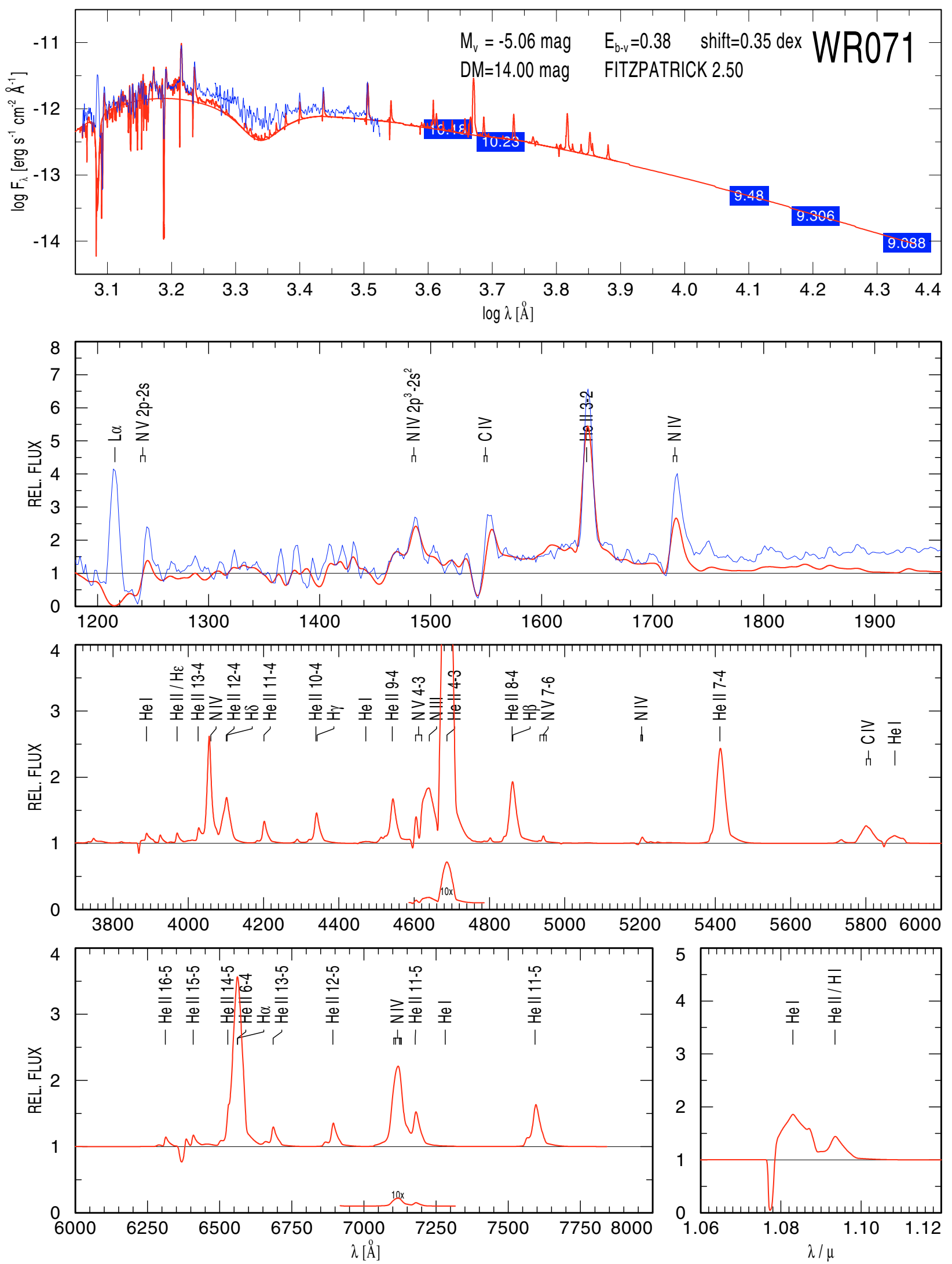

Fig. 42. Model: WNE 08-12, $T_{*}=56 \mathrm{kK}, \log \left(R_{\mathrm{t}} / R_{\odot}\right)=0.9$. 
W.-R. Hamann et al.: The Galactic WN stars, Online Material p 34

MODEL START 04/06/03 19:16:25 56234/0.7D/1600 L=5.3 N=1.5\% C=1E-4 Fe=1.4E-3 D4 WN-NODIEL 8-14 AFTER JOB N0.910
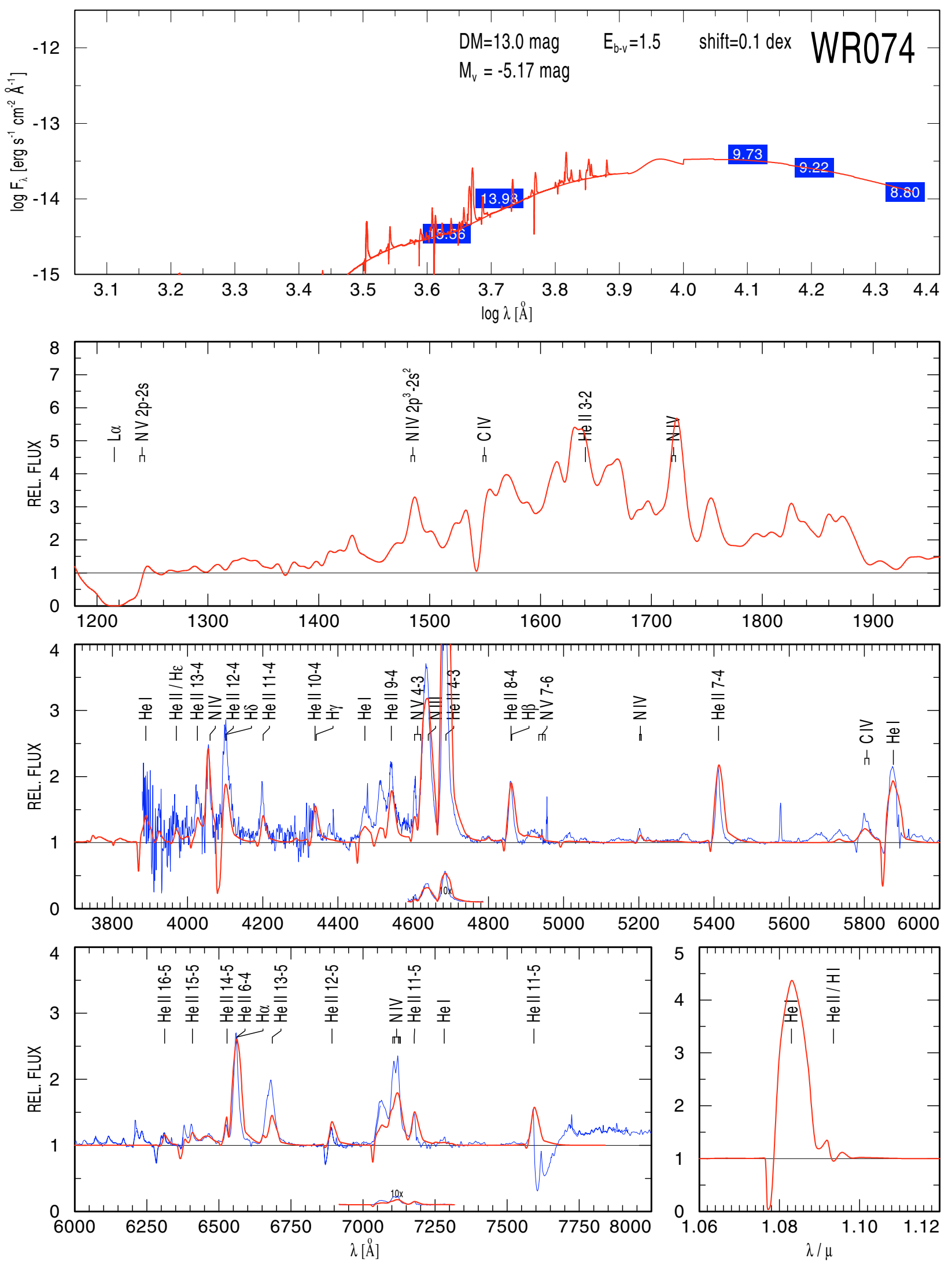

Fig. 43. Model: WNE 08-14, $T_{*}=56 \mathrm{kK}, \log \left(R_{\mathrm{t}} / R_{\odot}\right)=0.7$. 
W.-R. Hamann et al.: The Galactic WN stars, Online Material p 35

MODEL START 03/31/03 10:50:36 63096/0.6D/1600 L=5.3 N=1.5\% C=1E-4 Fe=1.4E-3 D4 WN-NODIEL 9-15 AFTER JOB NO.825
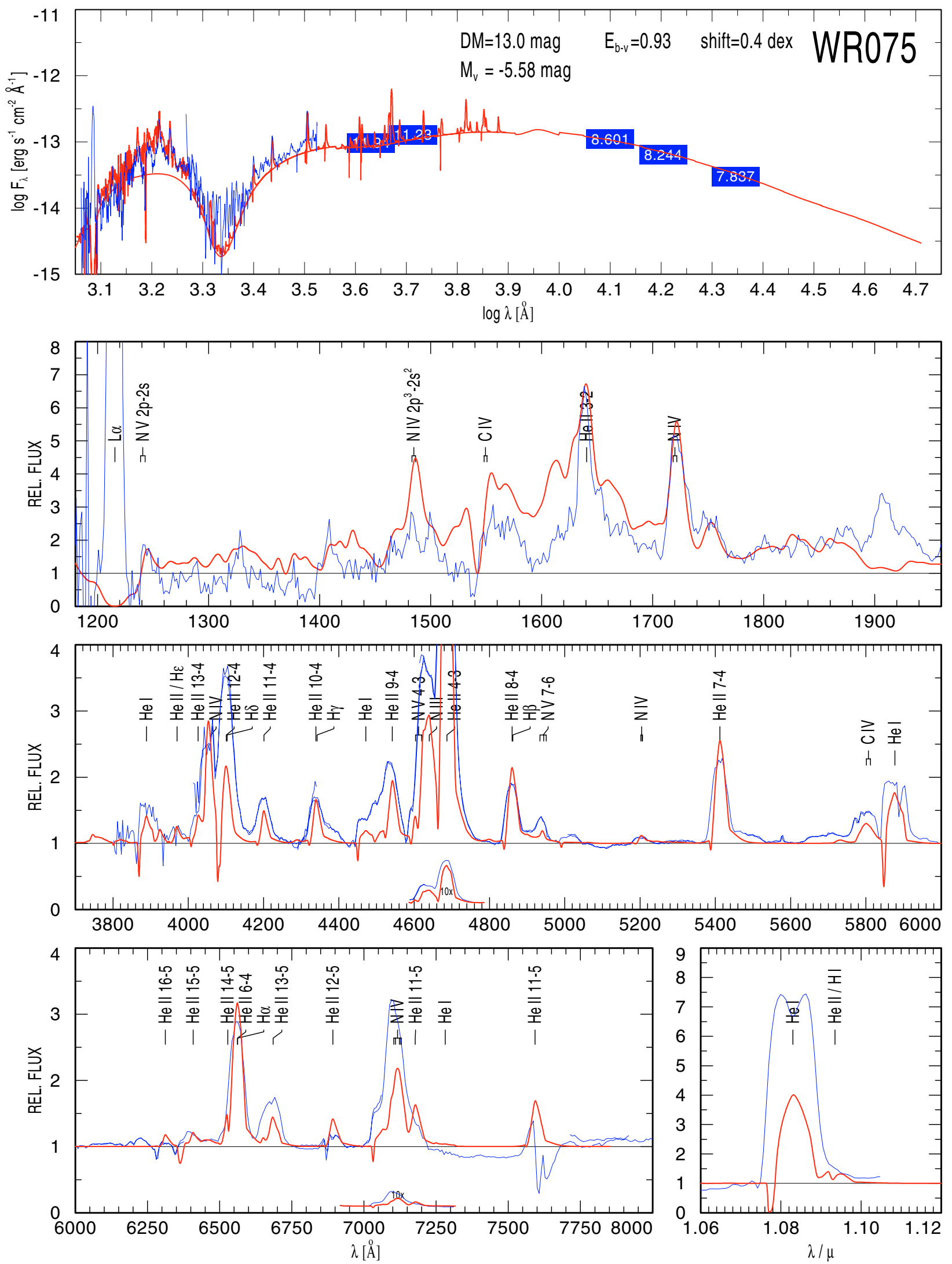

Fig. 44. Model: WNE 09-15, $T_{*}=63 \mathrm{kK}, \log \left(R_{\mathrm{t}} / R_{\odot}\right)=0.6$. 
W.-R. Hamann et al.: The Galactic WN stars, Online Material p 36

MODEL START 10/13/03 09:51:52 50119/1.0D/1000 L=5.3 H20 N1.5\% C1E-4 Fe0.14\% D4 WNL-NODR 07-11 AFTER JOB NO.610
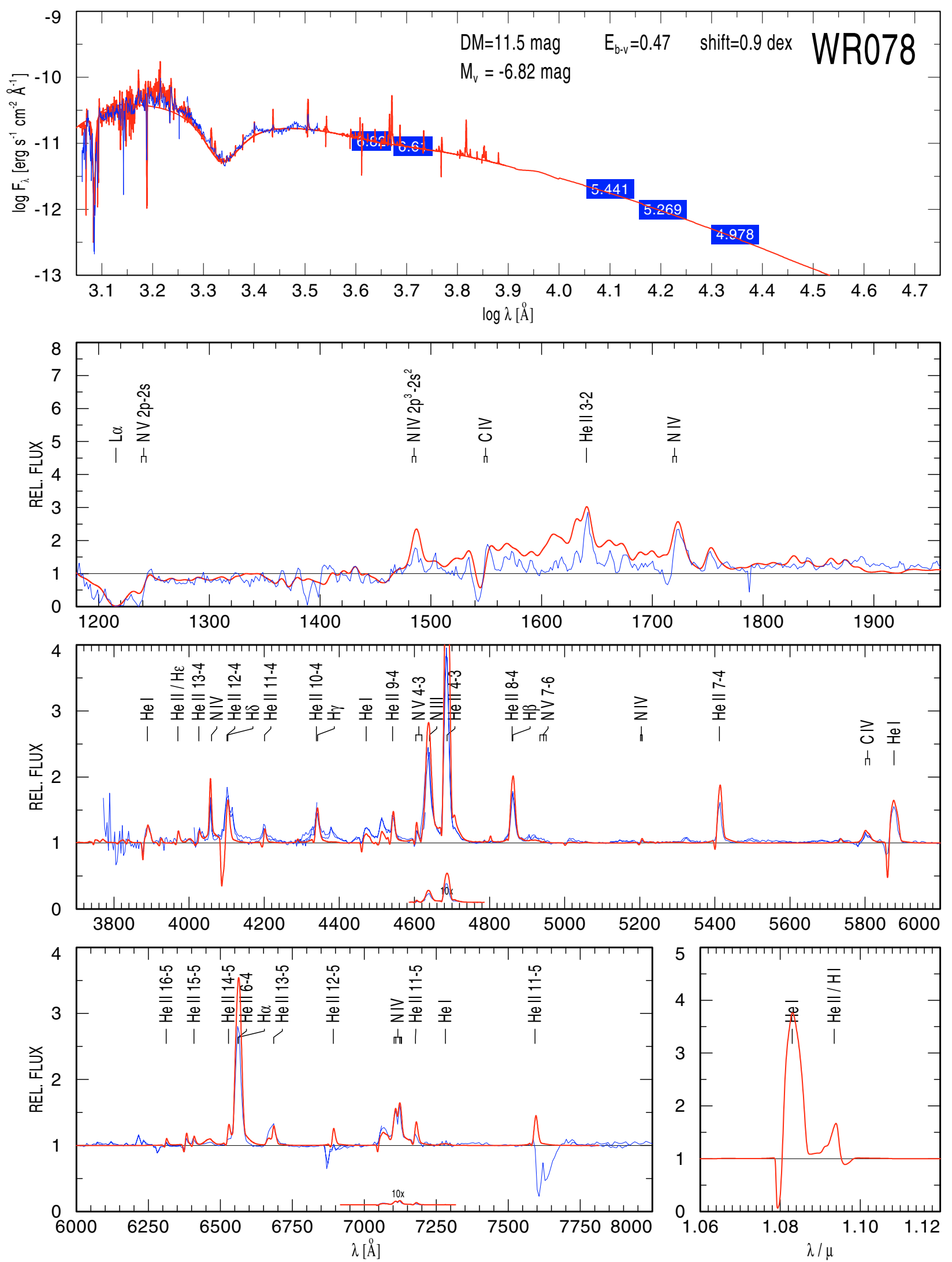

Fig. 45. Model: WNL 07-11, $T_{*}=50 \mathrm{kK}, \log \left(R_{\mathrm{t}} / R_{\odot}\right)=1.0$. 
W.-R. Hamann et al.: The Galactic WN stars, Online Material p 37

MODEL START 10/21/03 16:51:37 56234/0.7D/1000 L=5.3 H20 N1.5\% C1E-4 Fe0.14\% D4 WNL-NODR 8-14 AFTER JOB NO.649
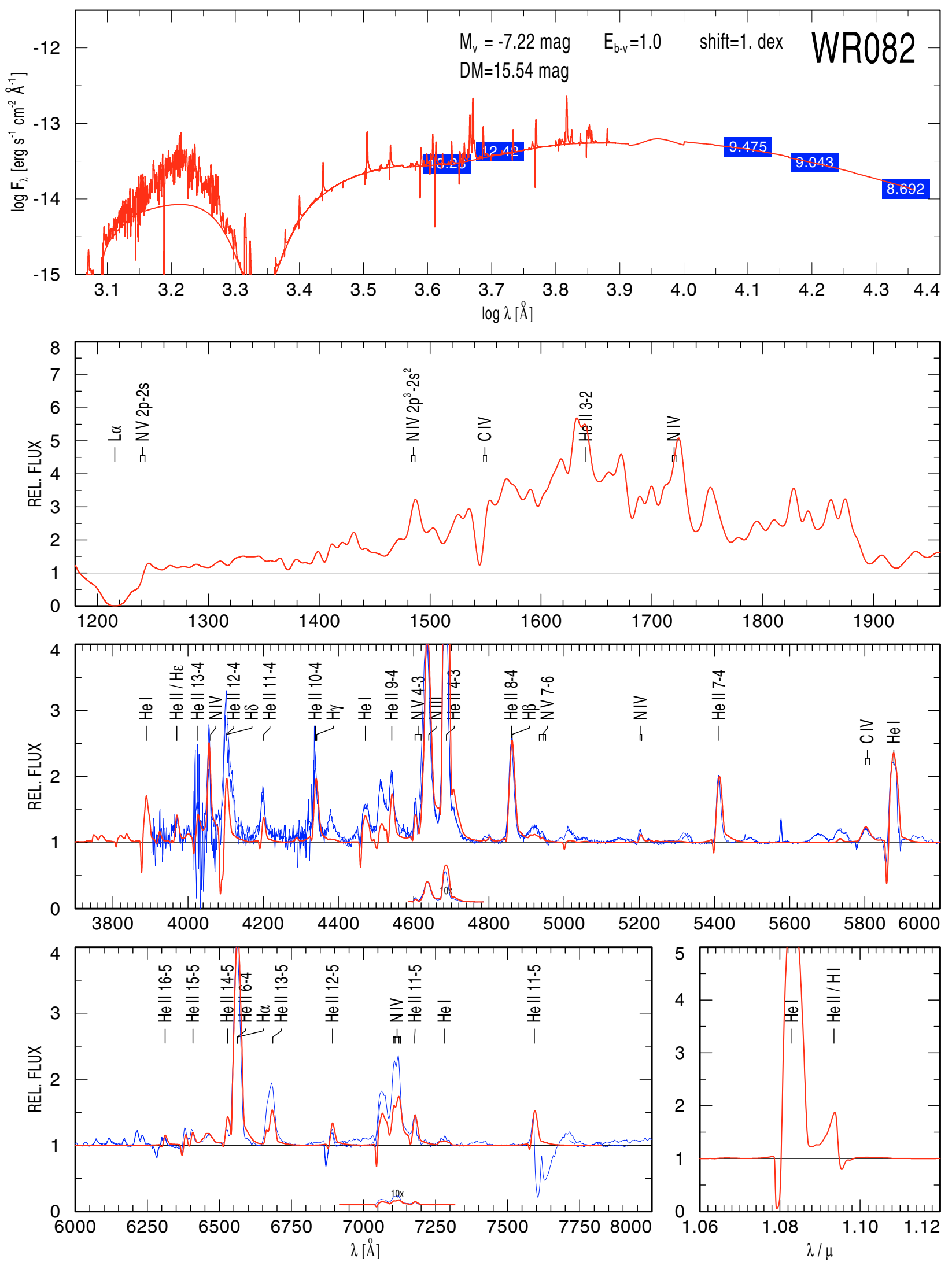

Fig. 46. Model: WNL 08-14, $T_{*}=56 \mathrm{kK}, \log \left(R_{\mathrm{t}} / R_{\odot}\right)=0.7$. 
W.-R. Hamann et al.: The Galactic WN stars, Online Material p 38

MODEL START 05/05/03 21:33:49 50119/0.90D/1600 L=5.3 N=1.5\% C=1E-4 Fe=1.4E-3 D4 WN-NODIEL 07-12 AFTER JOB NO.802
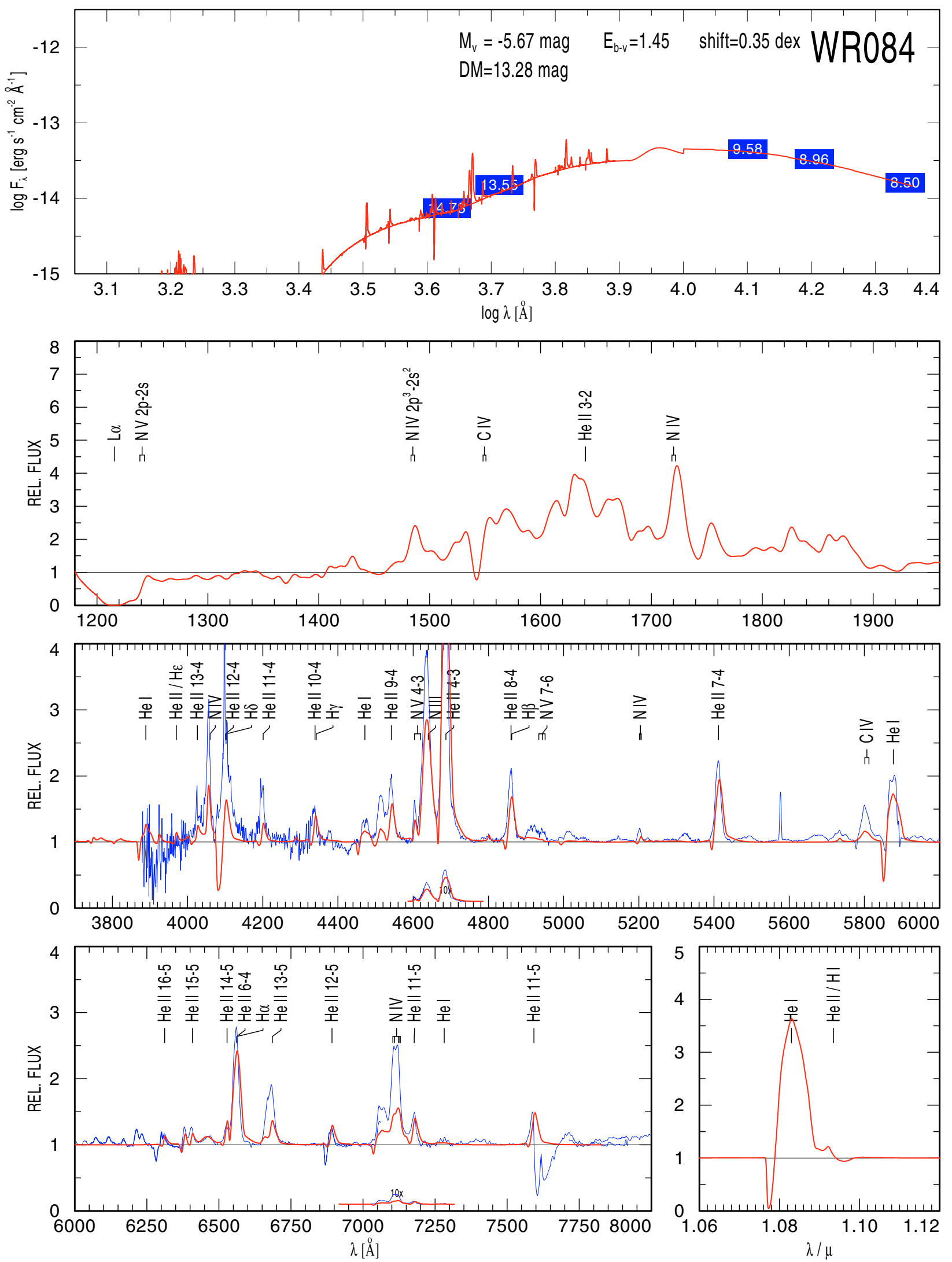

Fig. 47. Model: WNE 07-12, $T_{*}=50 \mathrm{kK}, \log \left(R_{\mathrm{t}} / R_{\odot}\right)=0.9$. 
W.-R. Hamann et al.: The Galactic WN stars, Online Material p 39

MODEL START 01/18/05 09:55:00 50119/1.1D/1000 L=5.3 H40 N1.5\% C1E-4 Fe0.14\% D4 WNL-NODR 07-10 AFTER JOB NO.276
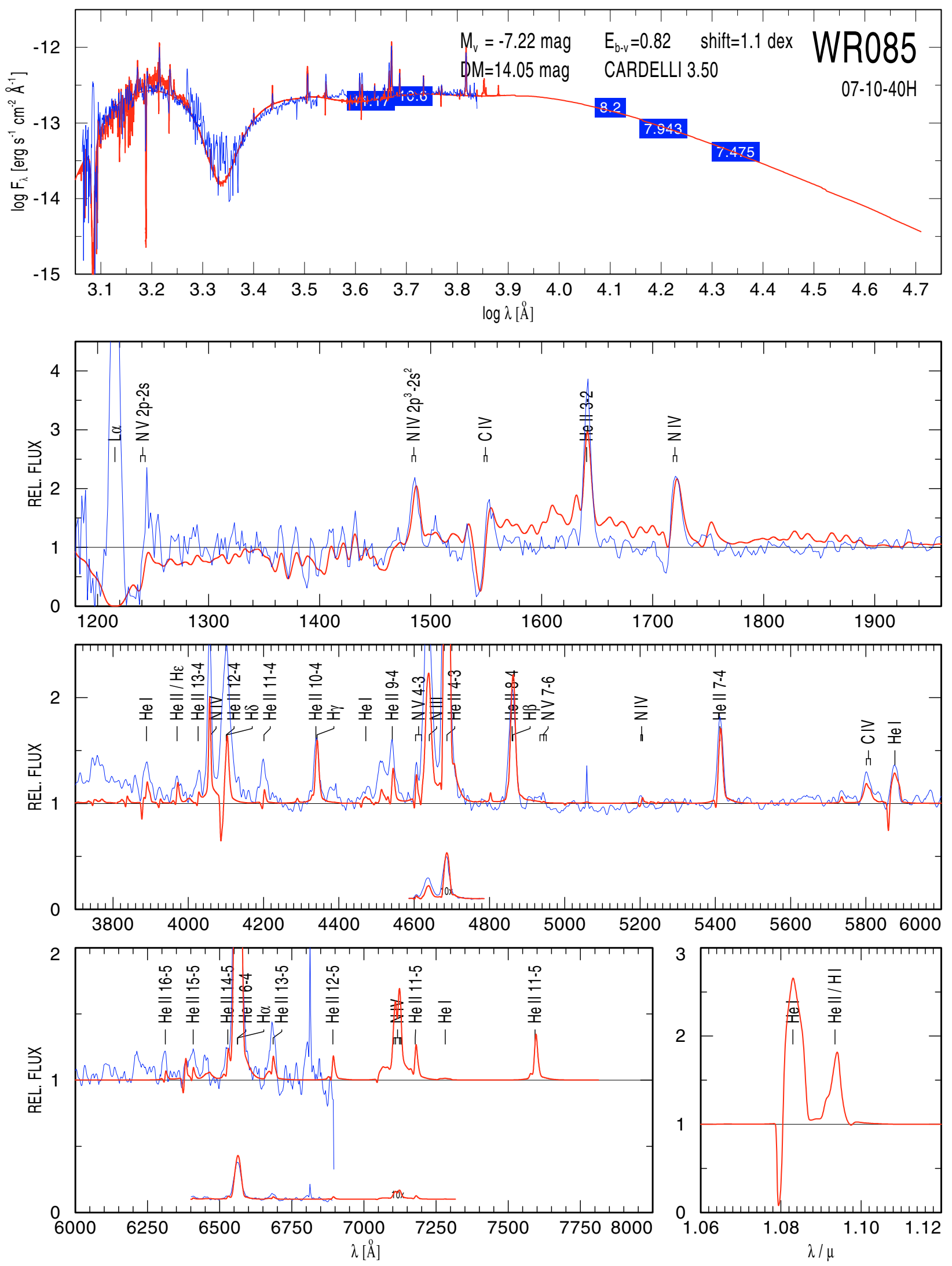

Fig. 48. Individual Model: $07-10, T_{*}=50 \mathrm{kK}, \log \left(R_{\mathrm{t}} / R_{\odot}\right)=1.1$ with $v_{\infty}=1000 \mathrm{~km} \mathrm{~s}^{-1}$ and $X_{\mathrm{H}}=0.40$. 
W.-R. Hamann et al.: The Galactic WN stars, Online Material p 40

MODEL START 11/21/03 16:50:35 44668/1.30D/1000 L5.3 H20 N1.5\% C1E-4 Fe0.14\% D4 WNL-NODR 06-08 AFTER JOB NO.156
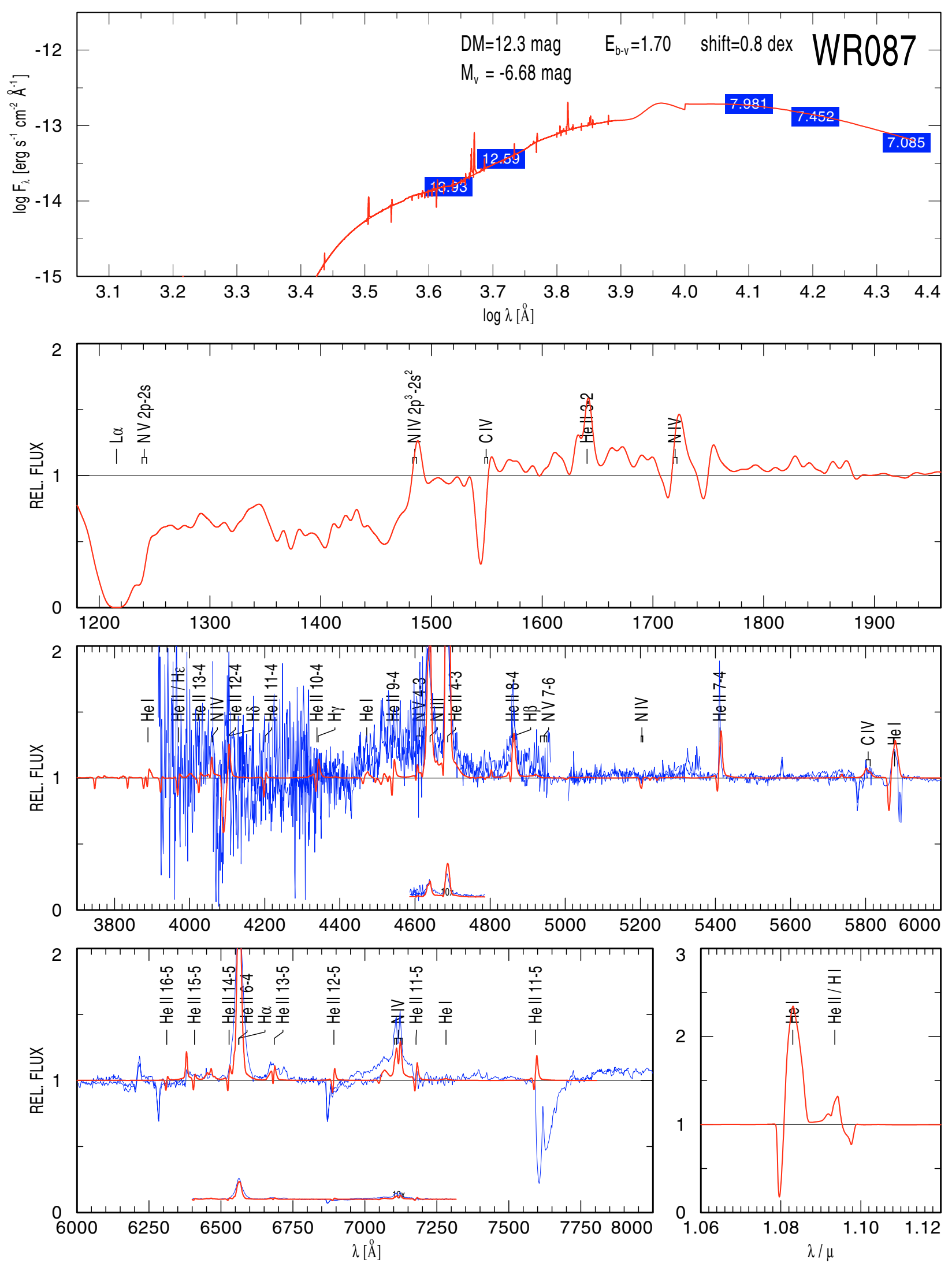

Fig. 49. Model: WNL 06-08, $T_{*}=45 \mathrm{kK}, \log \left(R_{\mathrm{t}} / R_{\odot}\right)=1.3$. 
W.-R. Hamann et al.: The Galactic WN stars, Online Material p 41

MODEL START 11/28/03 11:55:59 39811/1.40D/1000 L5.3 H20 N1.5\% C1E-4 Fe0.14\% D4 WNL-NODR 5-07 AFTER JOB NO.566
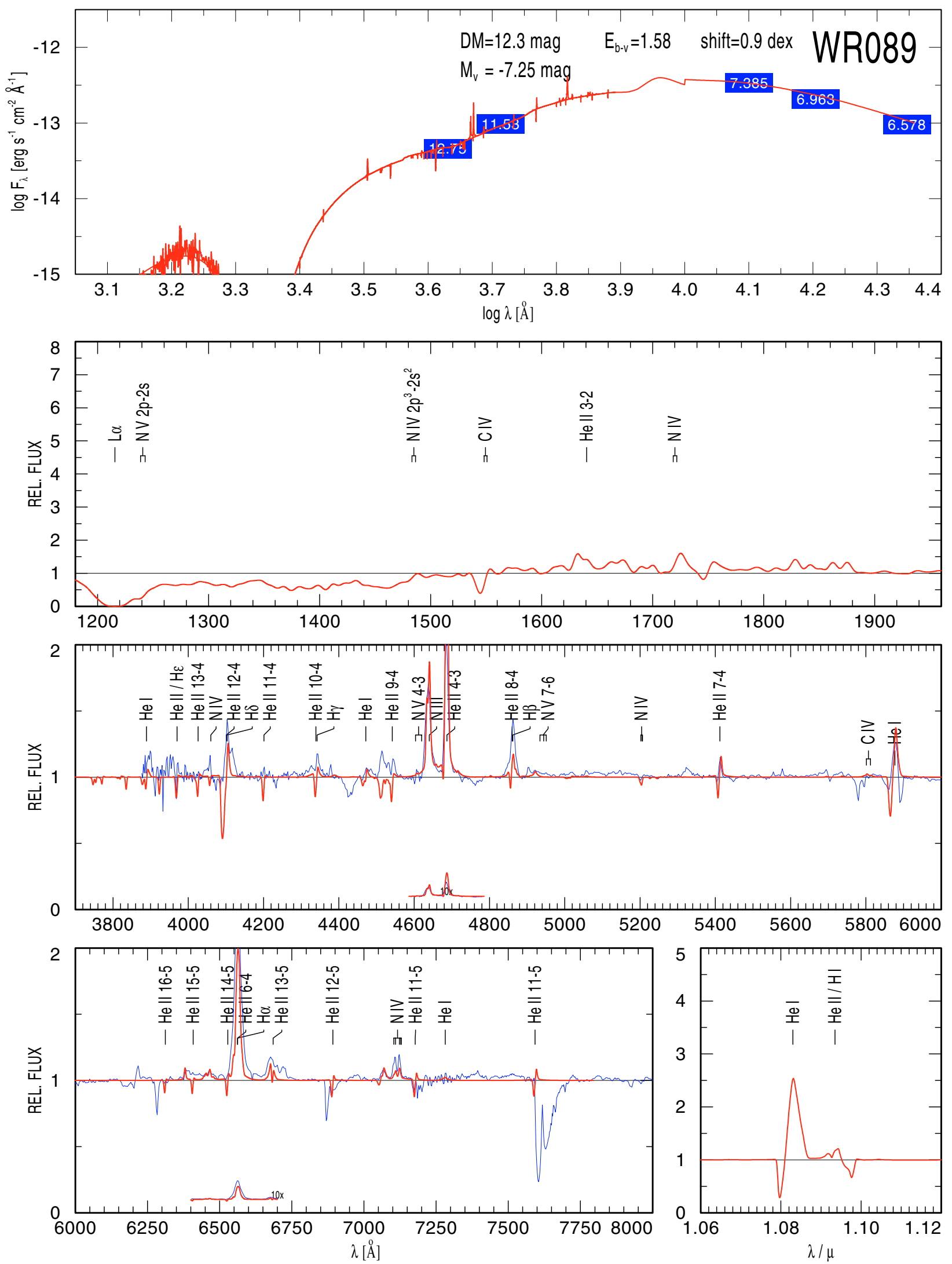

Fig. 50. Model: WNL 05- $07, T_{*}=40 \mathrm{kK}, \log \left(R_{\mathrm{t}} / R_{\odot}\right)=1.4$. 
W.-R. Hamann et al.: The Galactic WN stars, Online Material p 42

MODEL START 03/19/03 09:03:38 70795/0.4D/1600 L=5.3 N=1.5\% C=1E-4 Fe=1.4E-3 D4 WN-NODIEL 10-17 AFTER JOB NO.413
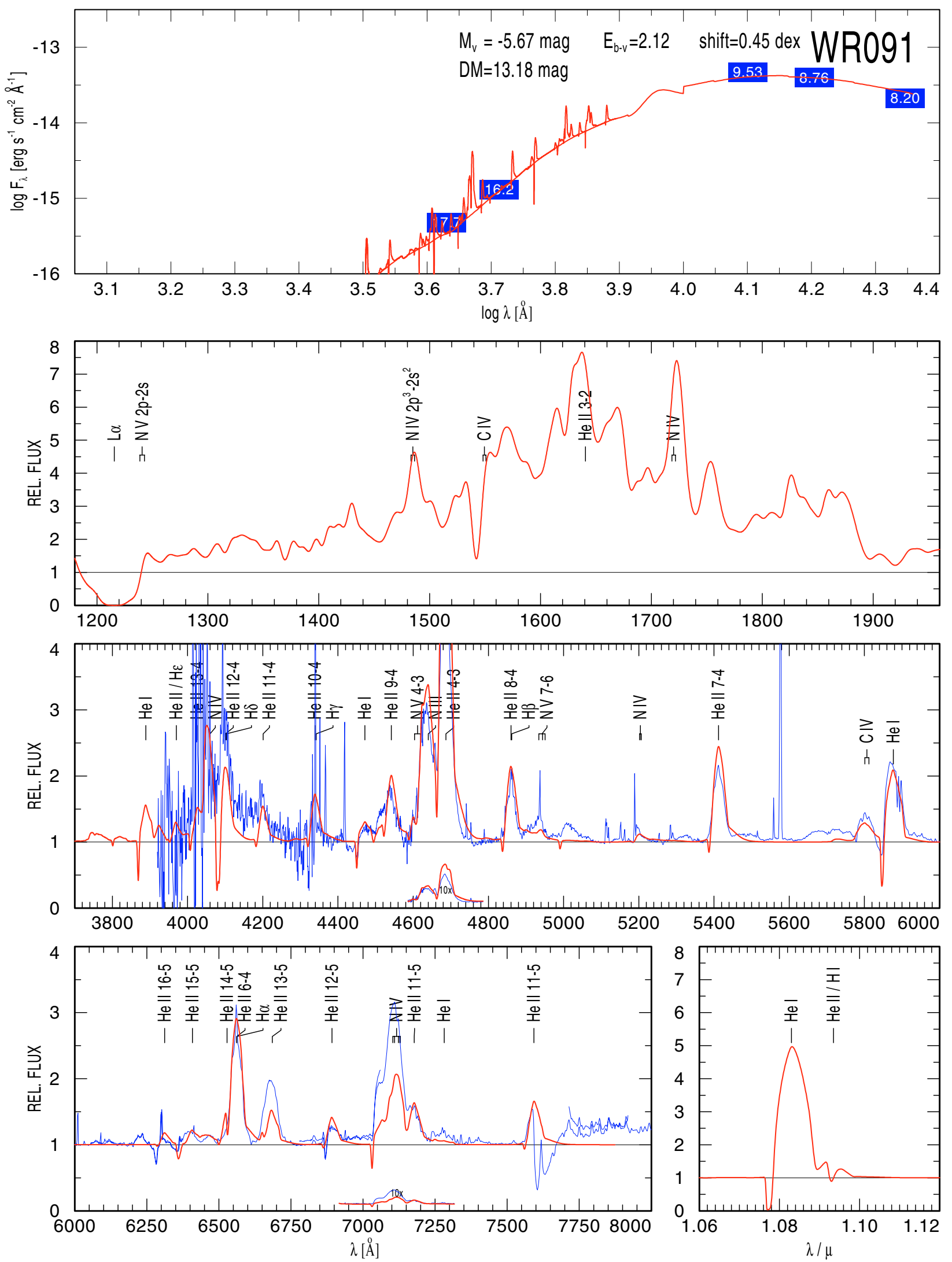

Fig. 51. Model: WNE $10-17, T_{*}=71 \mathrm{kK}, \log \left(R_{\mathrm{t}} / R_{\odot}\right)=0.4$. 
W.-R. Hamann et al.: The Galactic WN stars, Online Material p 43

MODEL START 04/20/03 00:18:54 56234/0.90D/1600 L=5.3 N=1.5\% C=1E-4 Fe=1.4E-3 D4 WN-NODIEL 08-12 AFTER JOB NO.172
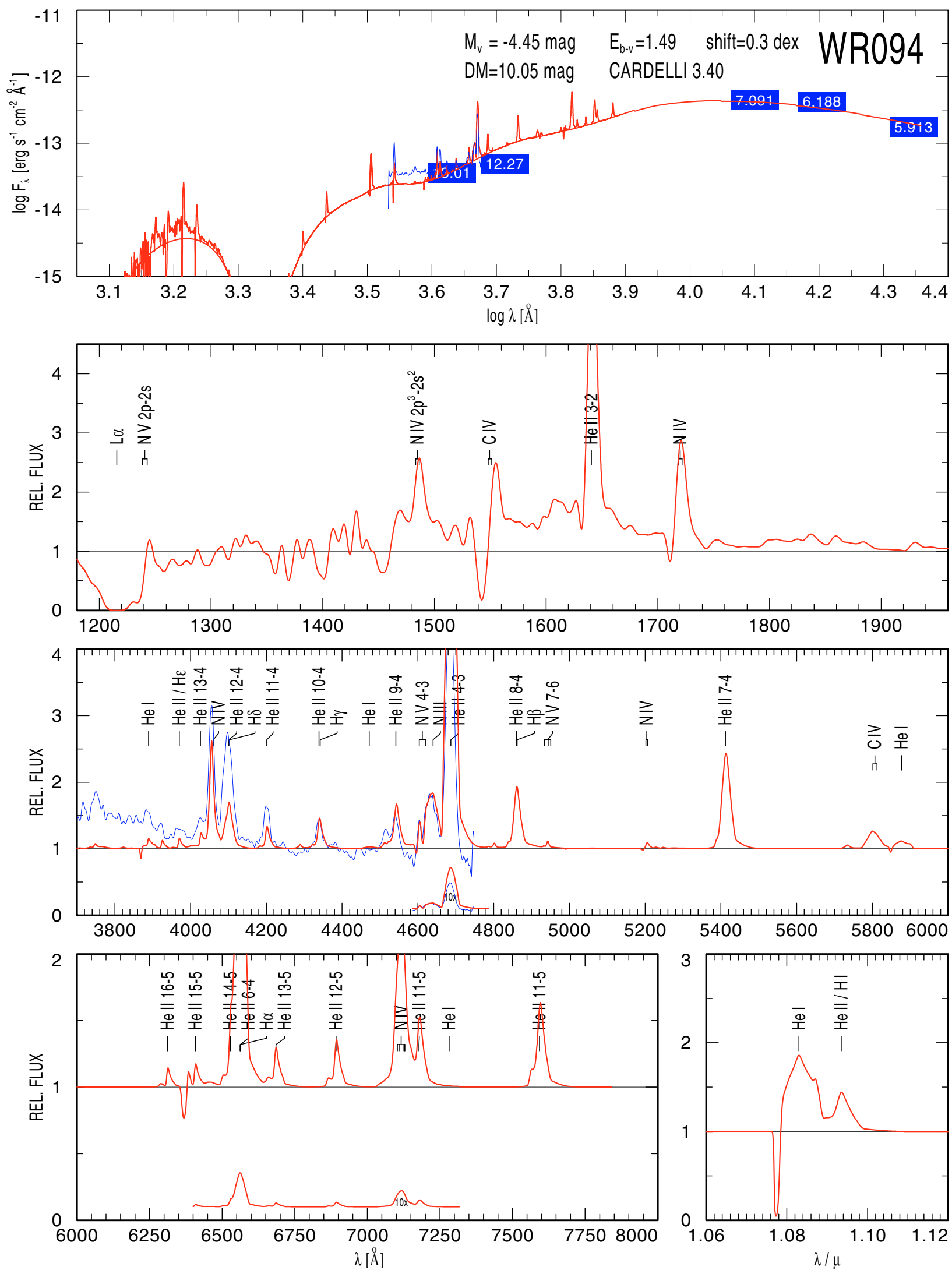

Fig. 52. Model: WNE 08-12, $T_{*}=56 \mathrm{kK}, \log \left(R_{\mathrm{t}} / R_{\odot}\right)=0.9$. 
W.-R. Hamann et al.: The Galactic WN stars, Online Material p 44

MODEL START 03/13/03 16:12:08 79433/0.3D/1600 L=5.3 N=1.5\% C=1E-4 Fe=1.4E-3 D4 WN-NODIEL 11-18 AFTER JOB NO.147
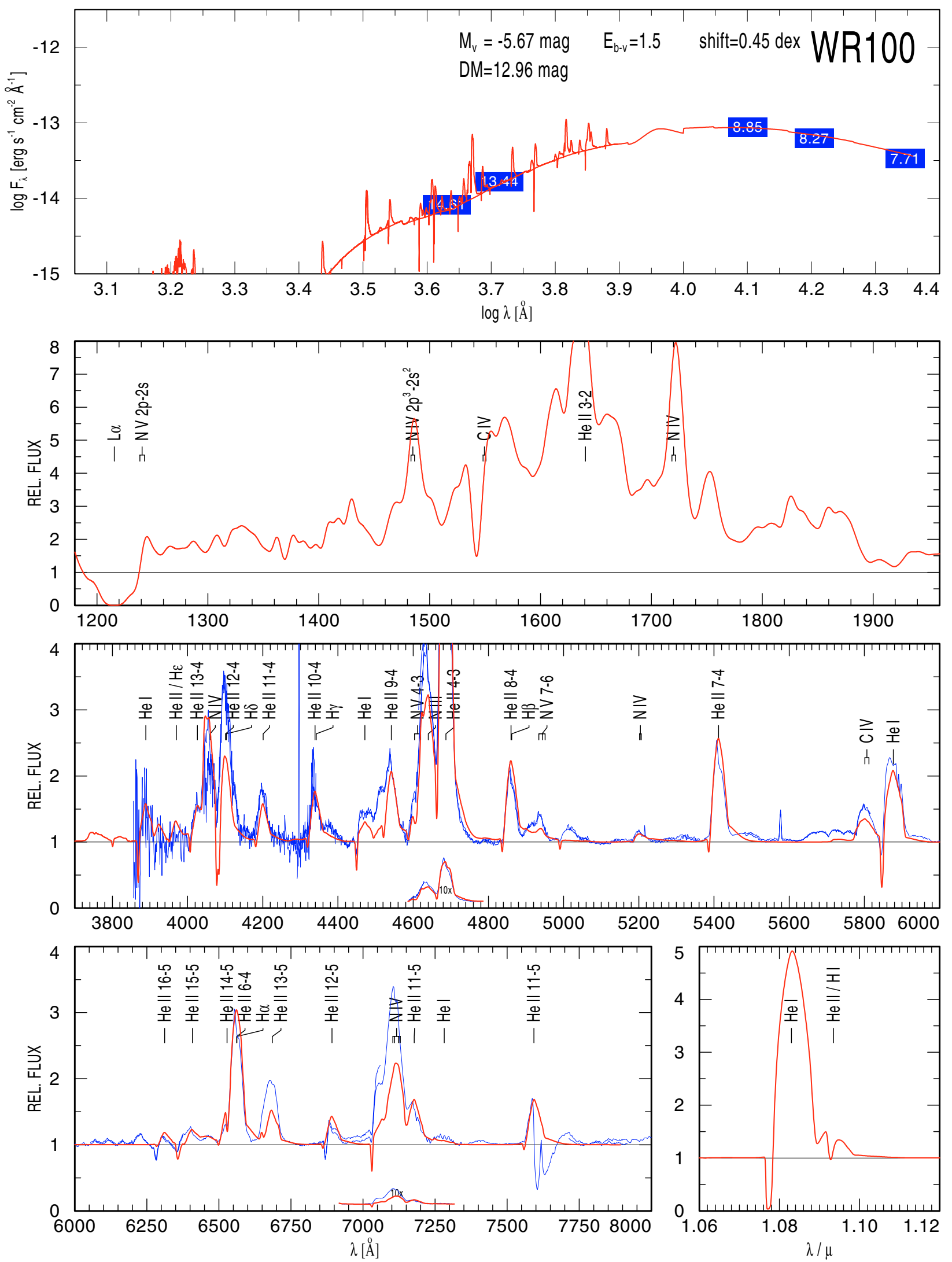

Fig. 53. Model: WNE $11-18, T_{*}=79 \mathrm{kK}, \log \left(R_{\mathrm{t}} / R_{\odot}\right)=0.3$. 
W.-R. Hamann et al.: The Galactic WN stars, Online Material p 45

MODEL START 12/08/03 10:01:14 35481/1.1D/1000 L5.3 H20 N1.5\% C1E-4 Fe0.14\% D4 WNL-NODR 04-10 AFTER JOB NO.454
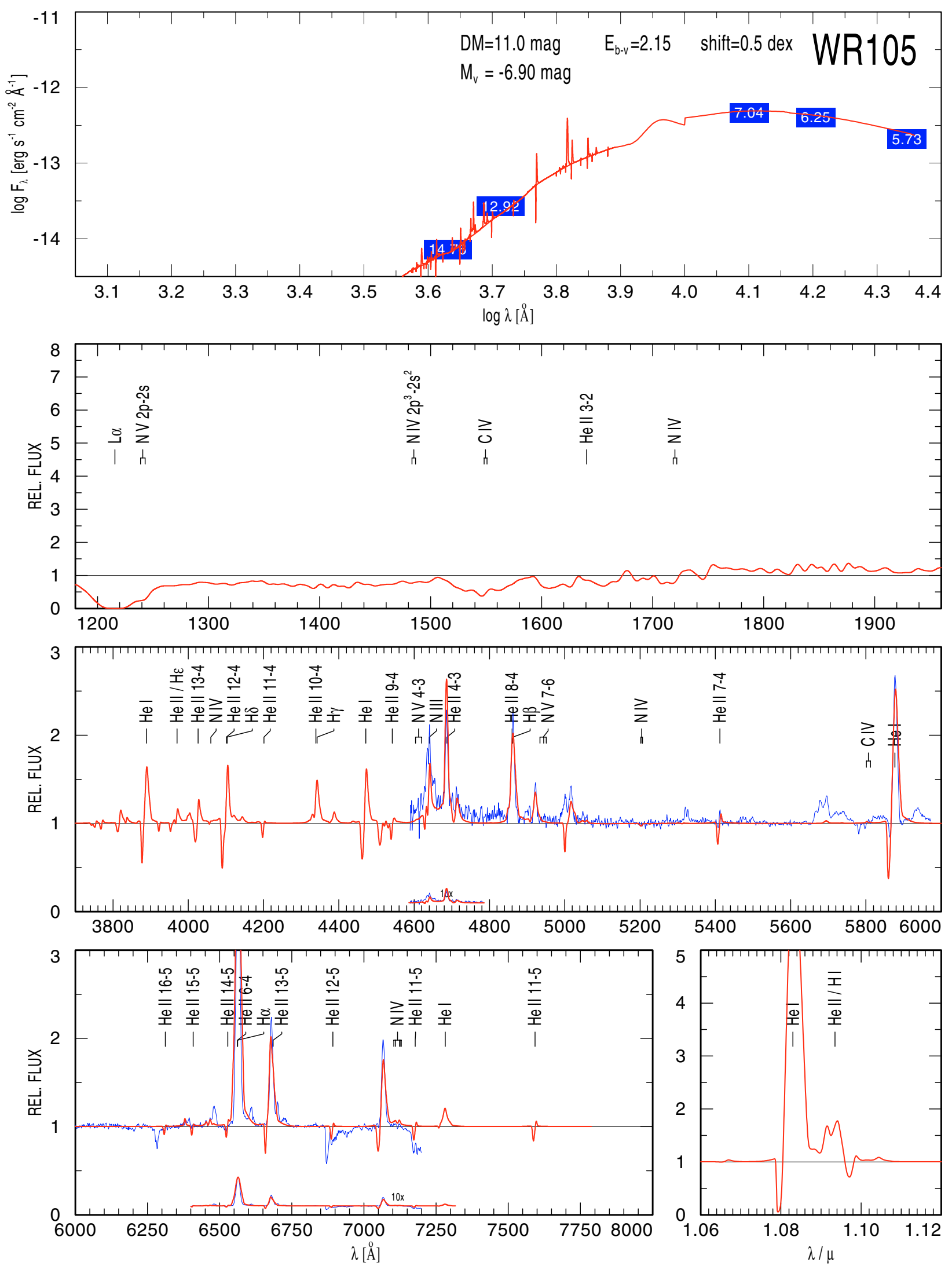

Fig. 54. Model: WNL 04-10, $T_{*}=35 \mathrm{kK}, \log \left(R_{\mathrm{t}} / R_{\odot}\right)=1.1$. 
W.-R. Hamann et al.: The Galactic WN stars, Online Material p 46

MODEL START 10/13/03 10:17:15 50119/0.80D/1000 L=5.3 H20 N1.5\% C1E-4 Fe0.14\% D4 WNL-NODR 07-13 AFTER JOB NO.438
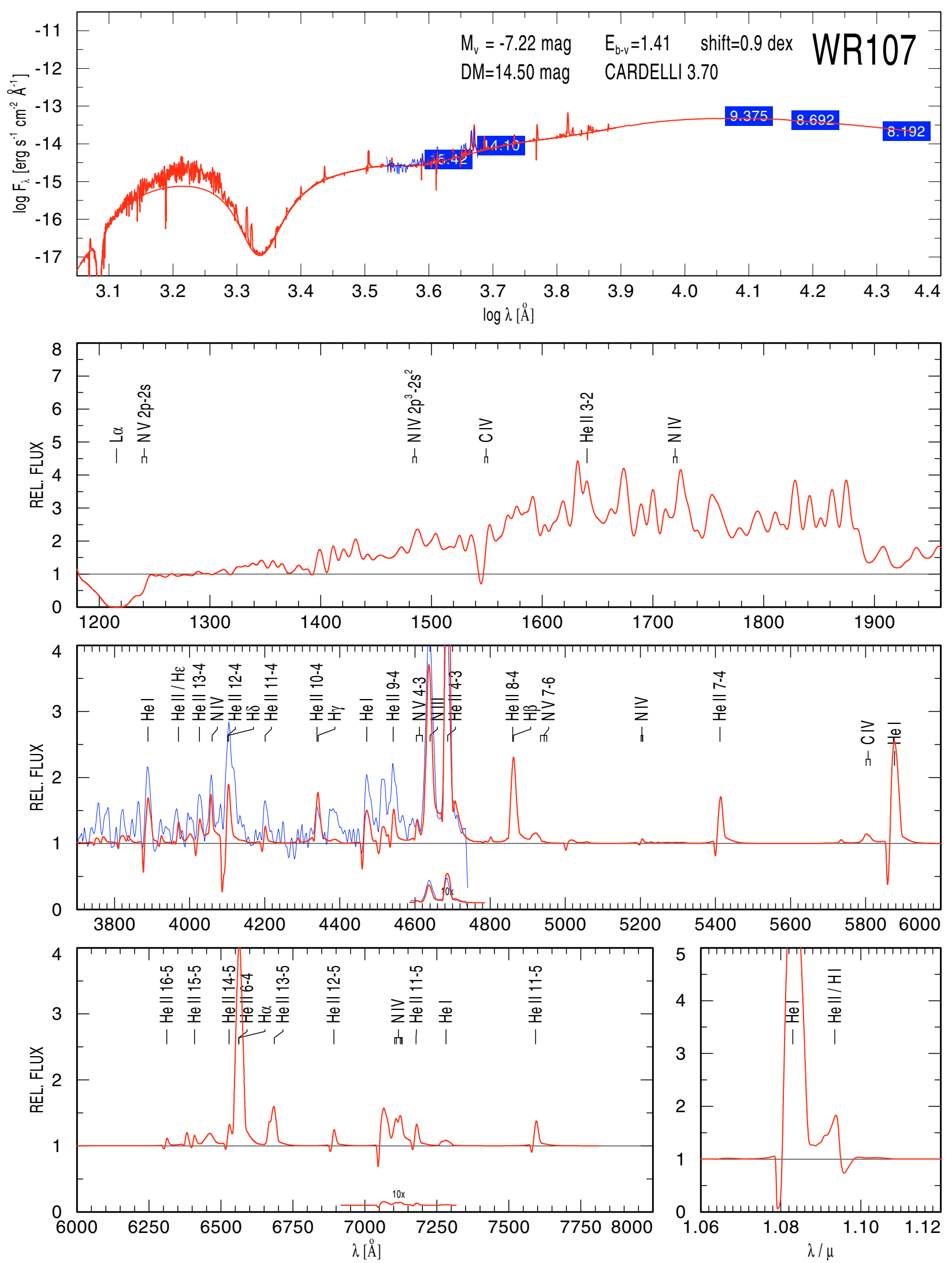

Fig. 55. Model: WNL 07-13, $T_{*}=50 \mathrm{kK}, \log \left(R_{\mathrm{t}} / R_{\odot}\right)=0.8$. 
W.-R. Hamann et al.: The Galactic WN stars, Online Material p 47

MODEL START 11/28/03 11:55:59 39811/1.40D/1000 L5.3 H20 N1.5\% C1E-4 Fe0.14\% D4 WNL-NODR 5-07 AFTER JOB N0.566
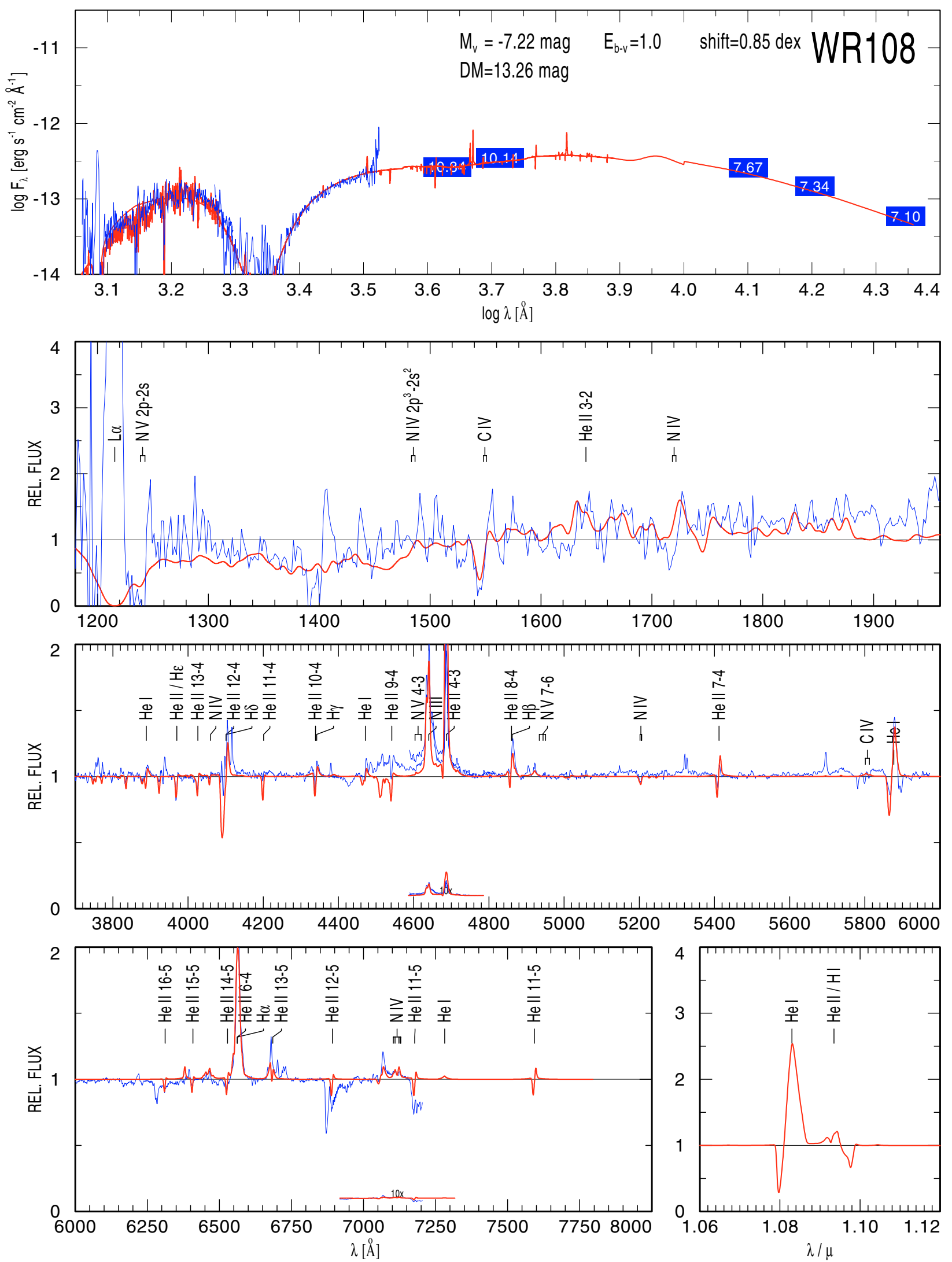

Fig. 56. Model: WNL 05-07, $T_{*}=40 \mathrm{kK}, \log \left(R_{\mathrm{t}} / R_{\odot}\right)=1.4$. 
W.-R. Hamann et al.: The Galactic WN stars, Online Material p 48

MODEL START 03/23/03 17:19:09 70795/0.5D/1600 L=5.3 N=1.5\% C=1E-4 Fe=1.4E-3 D4 WN-NODIEL 10-16 AFTER JOB N0.912
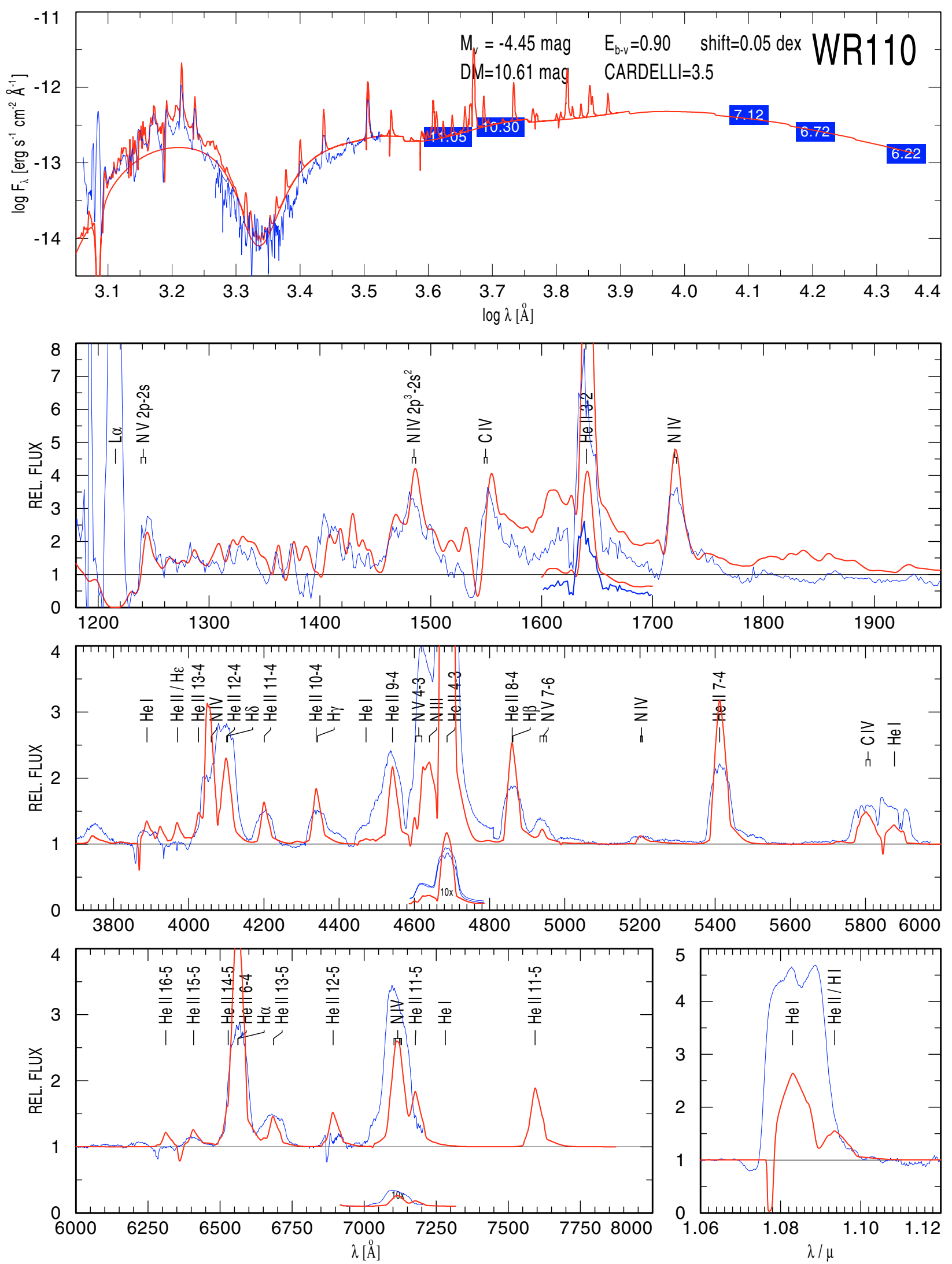

Fig. 57. Model: WNE 10-16, $T_{*}=71 \mathrm{kK}, \log \left(R_{\mathrm{t}} / R_{\odot}\right)=0.5$. 
W.-R. Hamann et al.: The Galactic WN stars, Online Material p 49

MODEL START 05/05/03 21:33:49 50119/0.90D/1600 L=5.3 N=1.5\% C=1E-4 Fe=1.4E-3 D4 WN-NODIEL 07-12 AFTER JOB NO.802
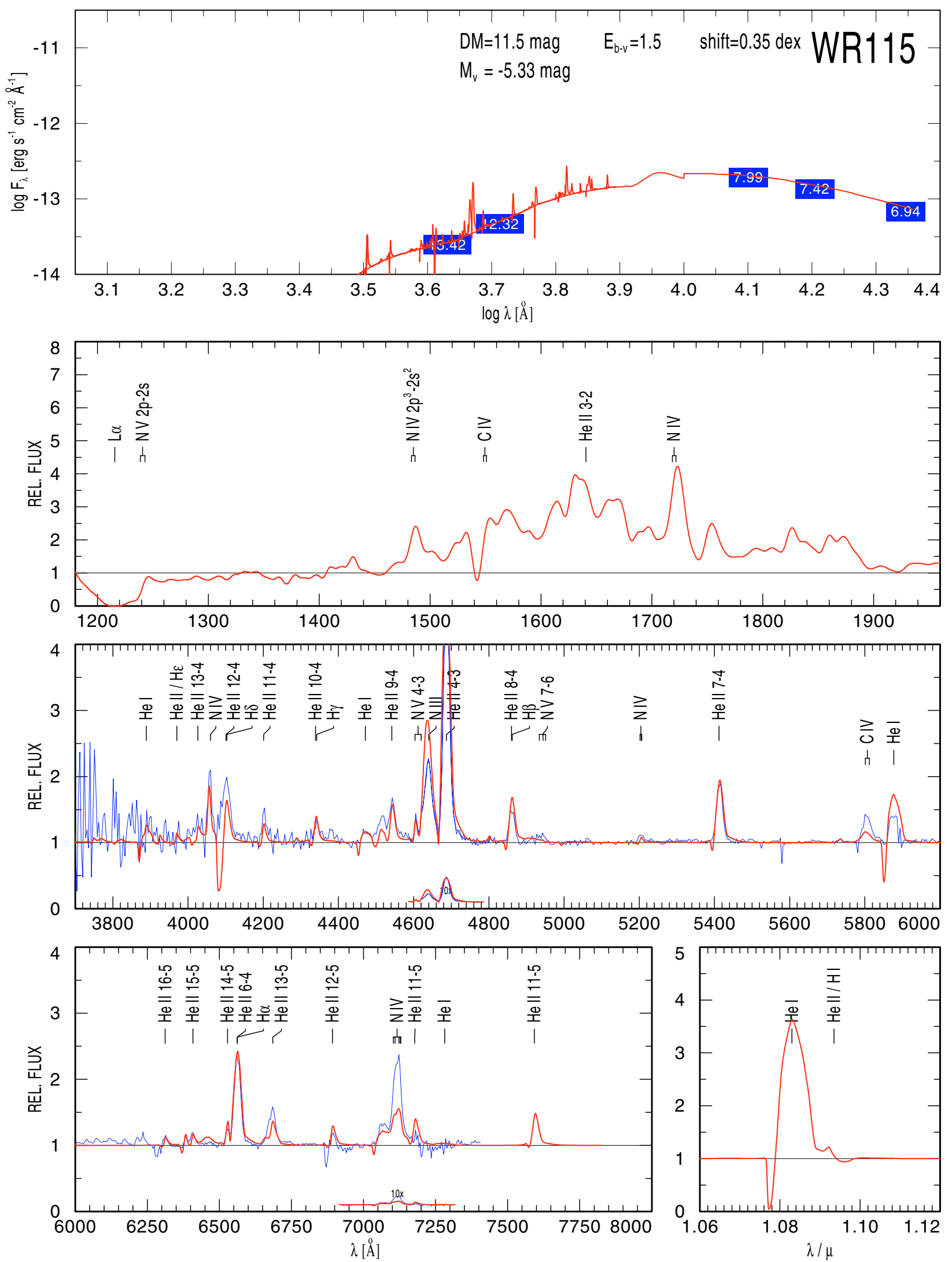

Fig. 58. Model: WNE 07-12, $T_{*}=50 \mathrm{kK}, \log \left(R_{\mathrm{t}} / R_{\odot}\right)=0.9$. 
W.-R. Hamann et al.: The Galactic WN stars, Online Material p 50

MODEL START 12/01/03 10:57:28 39811/0.80D/1000 L5.3 H20 N1.5\% C1E-4 Fe0.14\% D4 WNL-NODR 5-13 AFTER JOB NO.813
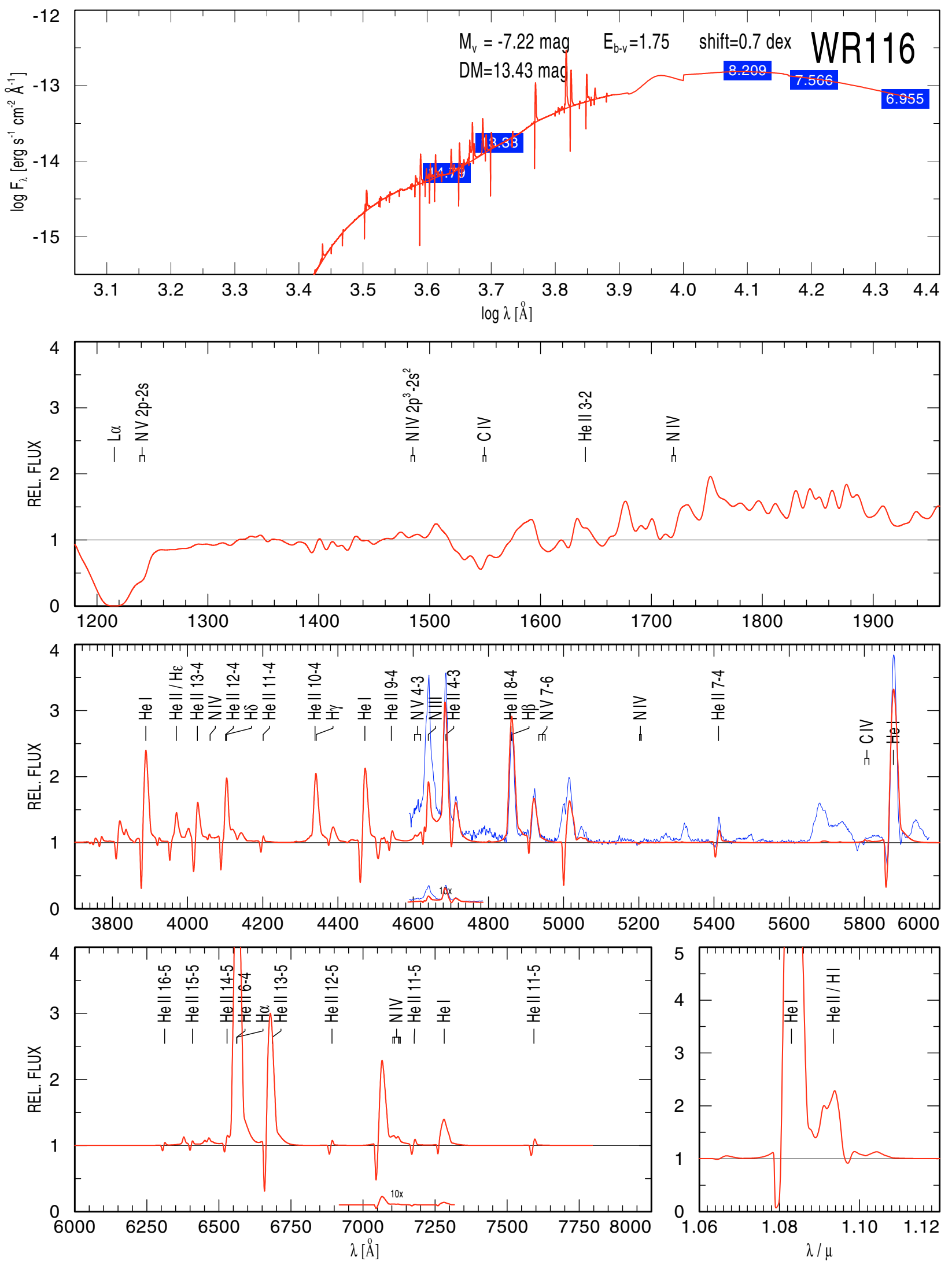

Fig. 59. Model: WNL 05-13, $T_{*}=40 \mathrm{kK}, \log \left(R_{\mathrm{t}} / R_{\odot}\right)=0.8$. 
W.-R. Hamann et al.: The Galactic WN stars, Online Material p 51

MODEL START 04/23/03 21:14:03 50119/0.80D/1600 L=5.3 N=1.5\% C=1E-4 Fe=1.4E-3 D4 WN-NODIEL 07-13 AFTER JOB NO.283
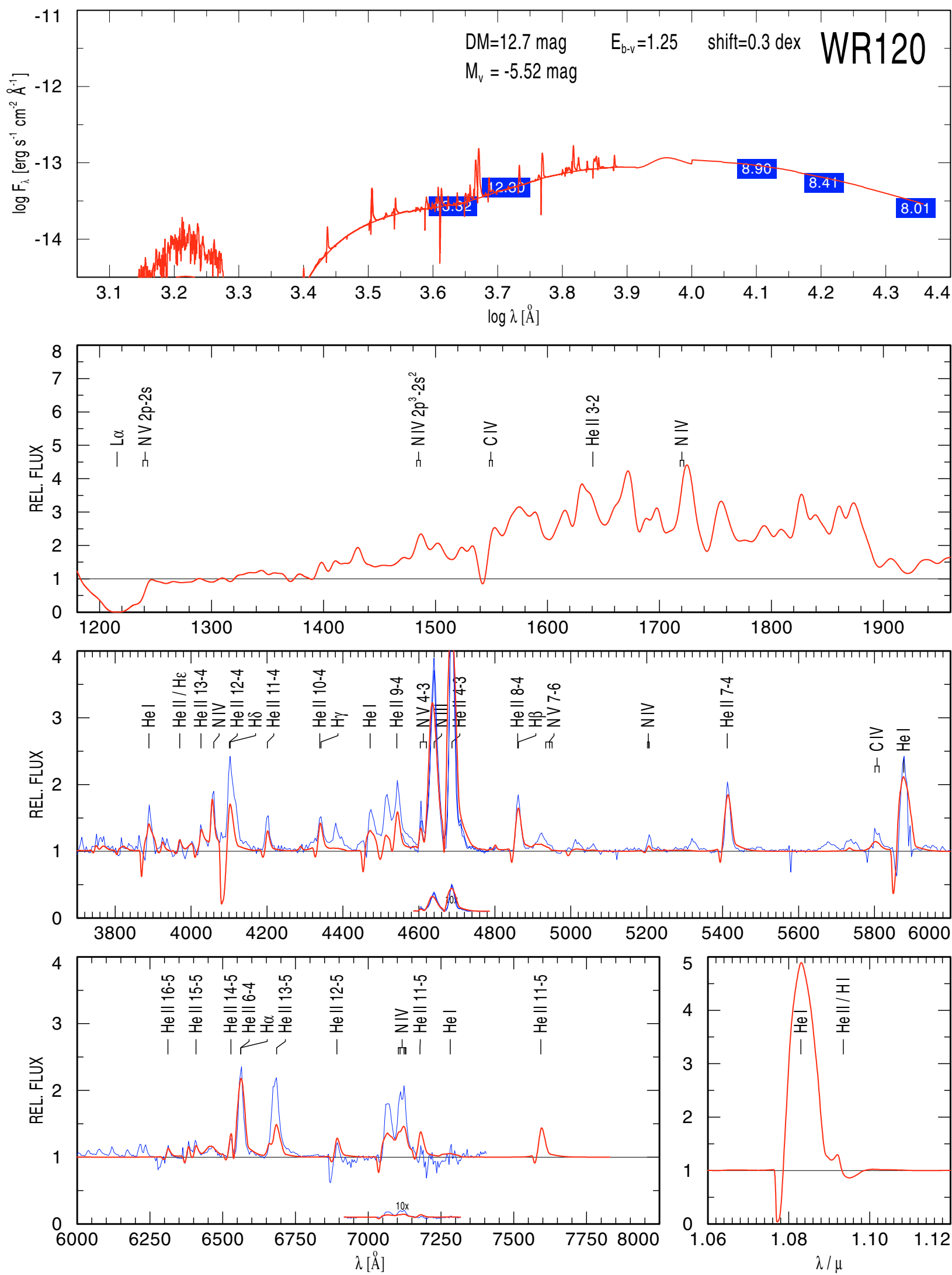

Fig. 60. Model: WNE 07-13, $T_{*}=50 \mathrm{kK}, \log \left(R_{\mathrm{t}} / R_{\odot}\right)=0.8$. 
W.-R. Hamann et al.: The Galactic WN stars, Online Material p 52

MODEL START 05/24/03 09:23:29 44668/0.70D/1600 L=5.3 N=1.5\% C=1E-4 Fe=1.4E-3 D4 WN-NODIEL 06-14 AFTER JOB NO.908
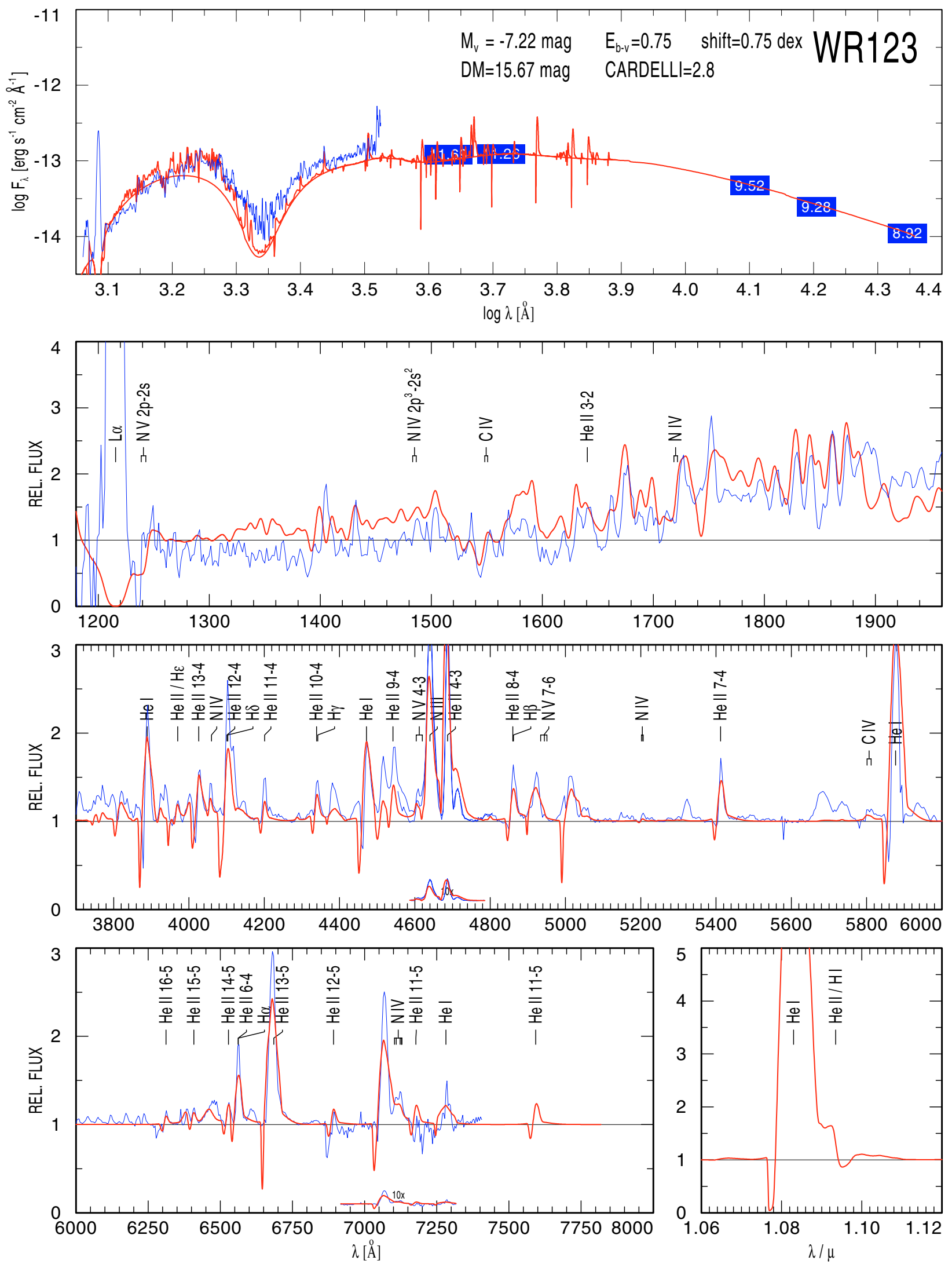

Fig. 61. Model: WNE 06-14, $T_{*}=45 \mathrm{kK}, \log \left(R_{\mathrm{t}} / R_{\odot}\right)=0.7$. 
W.-R. Hamann et al.: The Galactic WN stars, Online Material p 53

MODEL START 10/01/03 16:06:27 44668/0.70D/1000 L=5.3 H20 N1.5\% C1E-4 Fe0.14\% D4 WNL-NODR 06-14 AFTER JOB NO.514
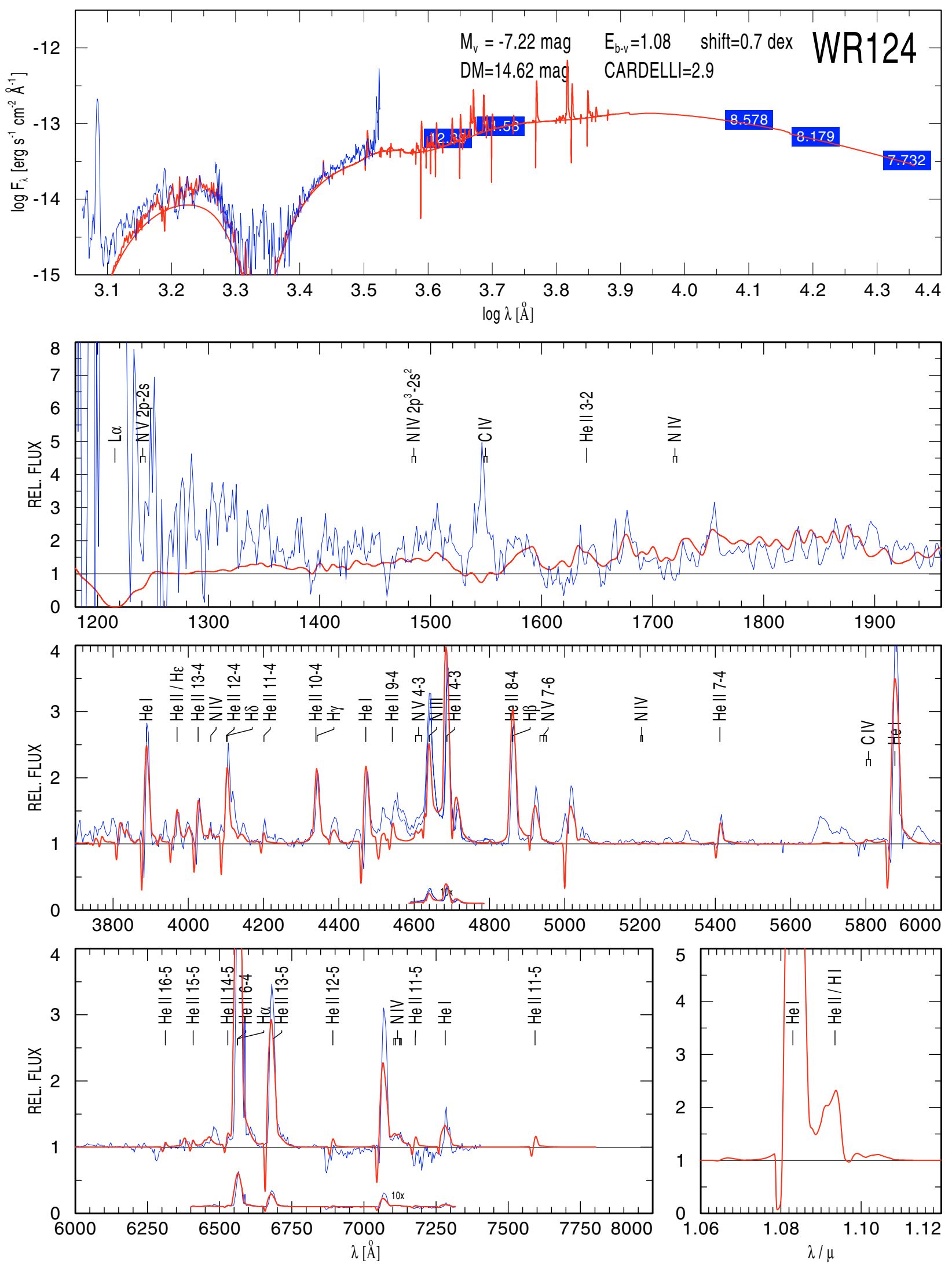

Fig. 62. Model: WNL 06-14, $T_{*}=45 \mathrm{kK}, \log \left(R_{\mathrm{t}} / R_{\odot}\right)=0.7$. 
W.-R. Hamann et al.: The Galactic WN stars, Online Material p 54

MODEL START 11/03/03 09:45:57 70795/1.1D/1000 L=5.3 H20 N1.5\% C1E-4 Fe0.14\% D4 WNL-NODR 10-10 AFTER JOB NO.631
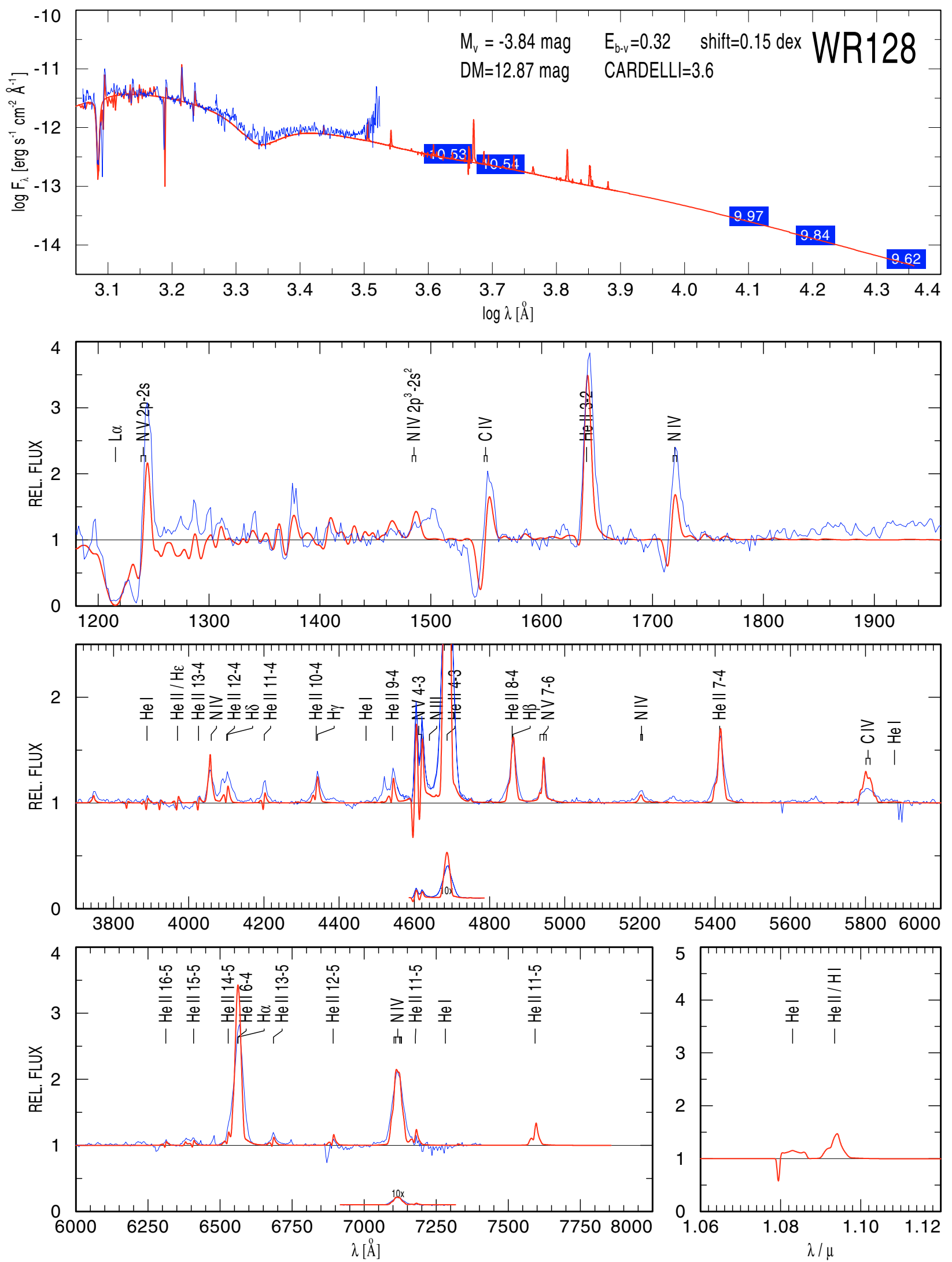

Fig. 63. Model: WNL $10-10, T_{*}=71 \mathrm{kK}, \log \left(R_{\mathrm{t}} / R_{\odot}\right)=1.1$. 
W.-R. Hamann et al.: The Galactic WN stars, Online Material p 55

MODEL START 04/04/03 12:29:14 63096/0.9D/1600 L=5.3 N=1.5\% C=1E-4 Fe=1.4E-3 D4 WN-NODIEL 09-12 AFTER JOB NO.946
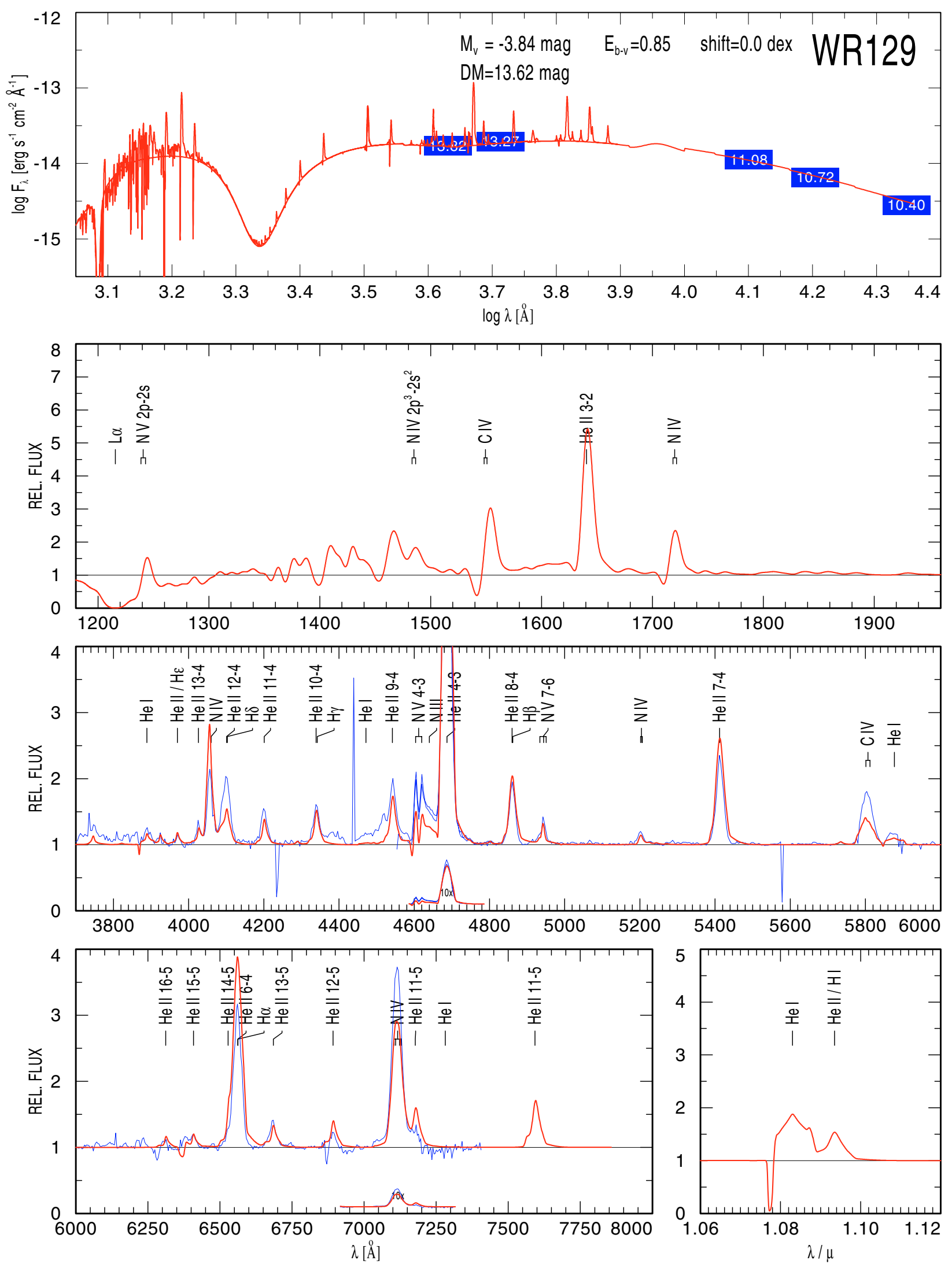

Fig. 64. Model: WNE 09-12, $T_{*}=63 \mathrm{kK}, \log \left(R_{\mathrm{t}} / R_{\odot}\right)=0.9$. 
W.-R. Hamann et al.: The Galactic WN stars, Online Material p 56

MODEL START 11/24/03 09:56:15 44668/1.0D/1000 L5.3 H20 N1.5\% C1E-4 Fe0.14\% D4 WNL-NODR 06-11 AFTER JOB N0.727
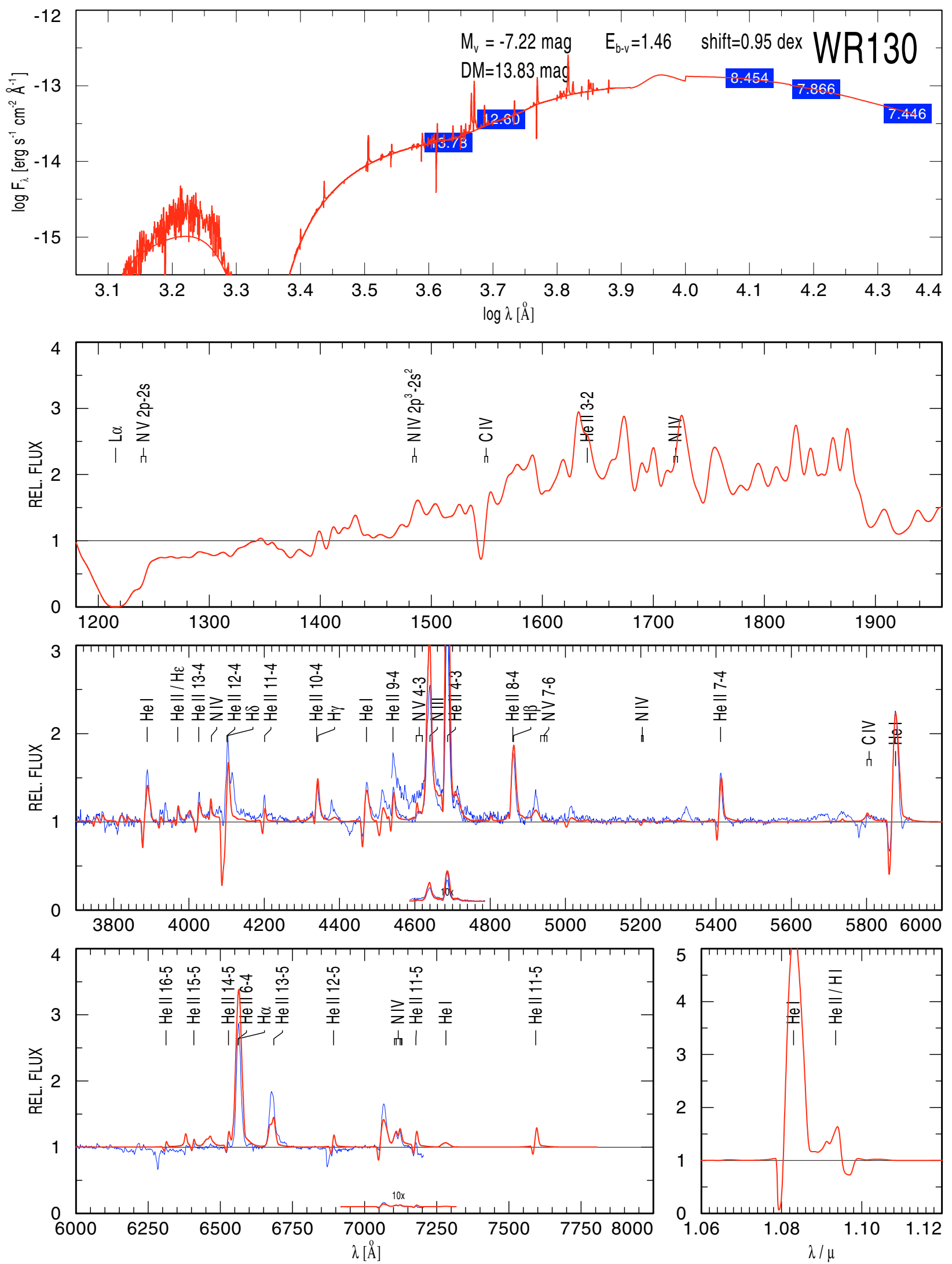

Fig. 65. Model: WNL 06-11, $T_{*}=45 \mathrm{kK}, \log \left(R_{\mathrm{t}} / R_{\odot}\right)=1.0$. 
W.-R. Hamann et al.: The Galactic WN stars, Online Material p 57

MODEL START 11/21/03 16:50:35 44668/1.30D/1000 L5.3 H20 N1.5\% C1E-4 Fe0.14\% D4 WNL-NODR 06-08 AFTER JOB N0.156
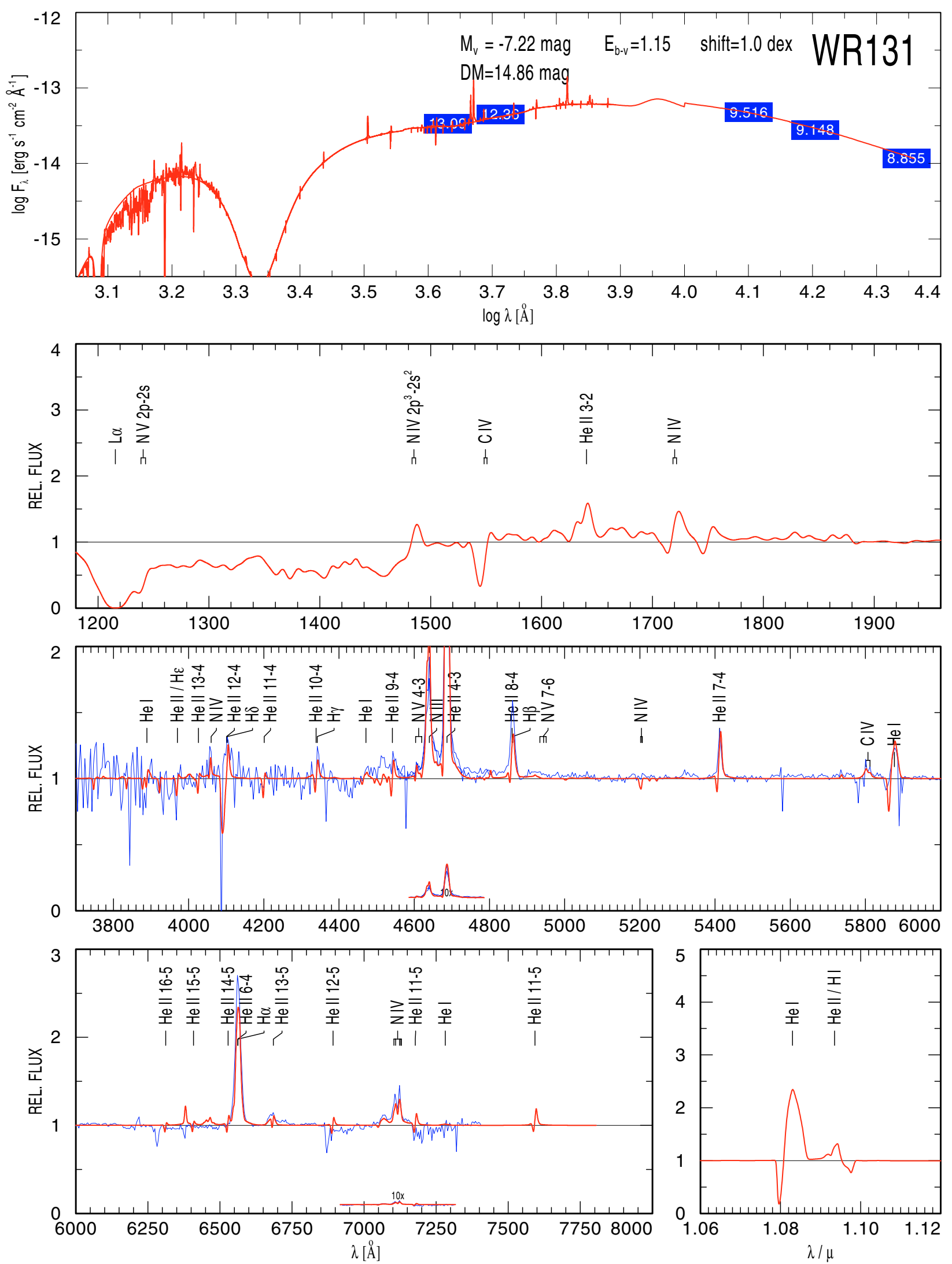

Fig. 66. Model: WNL 06-08, $T_{*}=45 \mathrm{kK}, \log \left(R_{\mathrm{t}} / R_{\odot}\right)=1.3$. 
W.-R. Hamann et al.: The Galactic WN stars, Online Material p 58

MODEL START 03/31/03 23:18:48 63096/0.7D/1600 L=5.3 N=1.5\% C=1E-4 Fe=1.4E-3 D4 WN-NODIEL 9-14 AFTER JOB NO.425
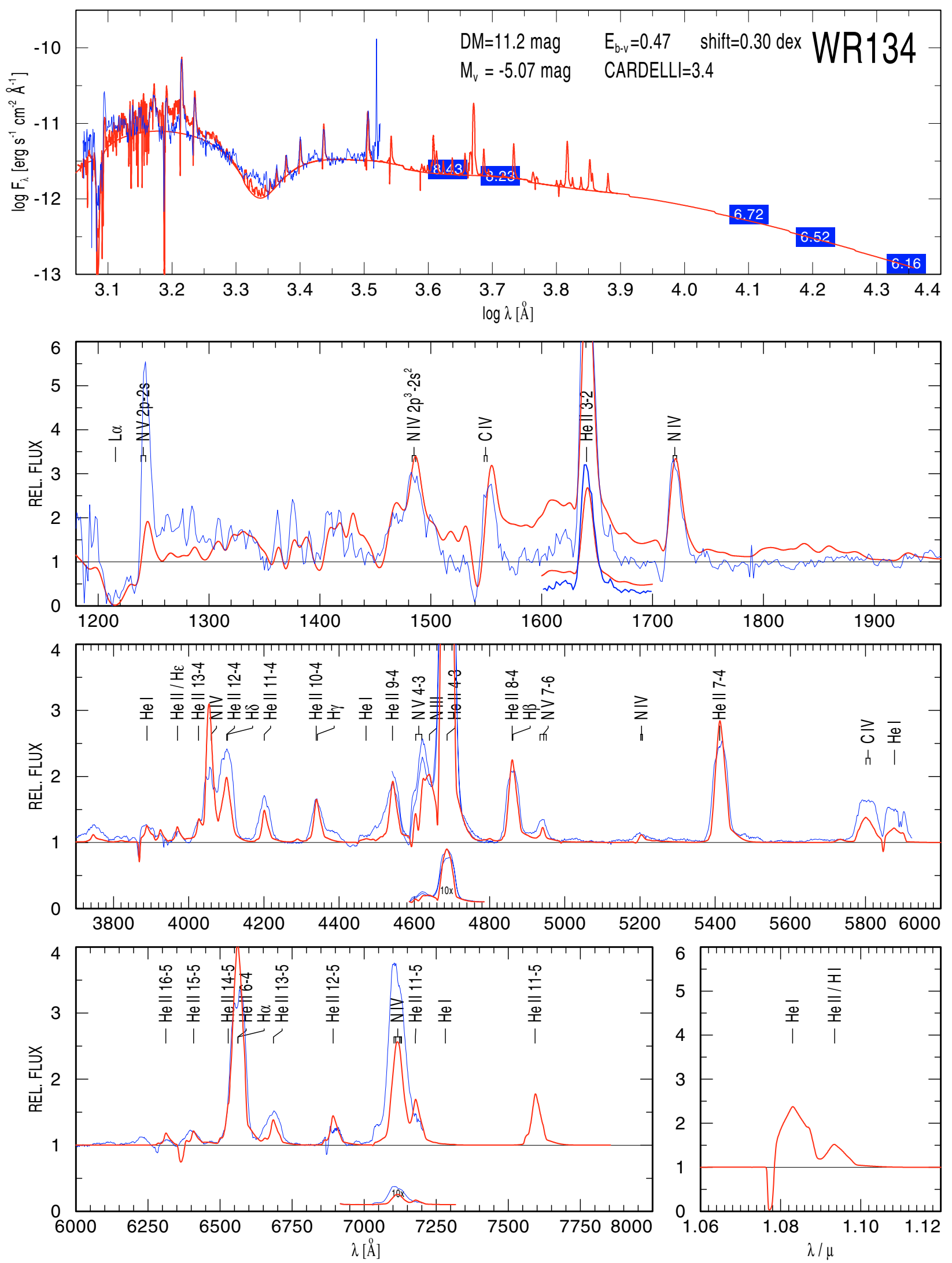

Fig. 67. Model: WNE 09-14, $T_{*}=63 \mathrm{kK}, \log \left(R_{\mathrm{t}} / R_{\odot}\right)=0.7$. 
W.-R. Hamann et al.: The Galactic WN stars, Online Material p 59

MODEL START 11/05/03 09:21:16 70795/0.5D/1000 L=5.3 H20 N1.5\% C1E-4 Fe0.14\% D4 WNL-NODR 10-16 AFTER JOB NO.368
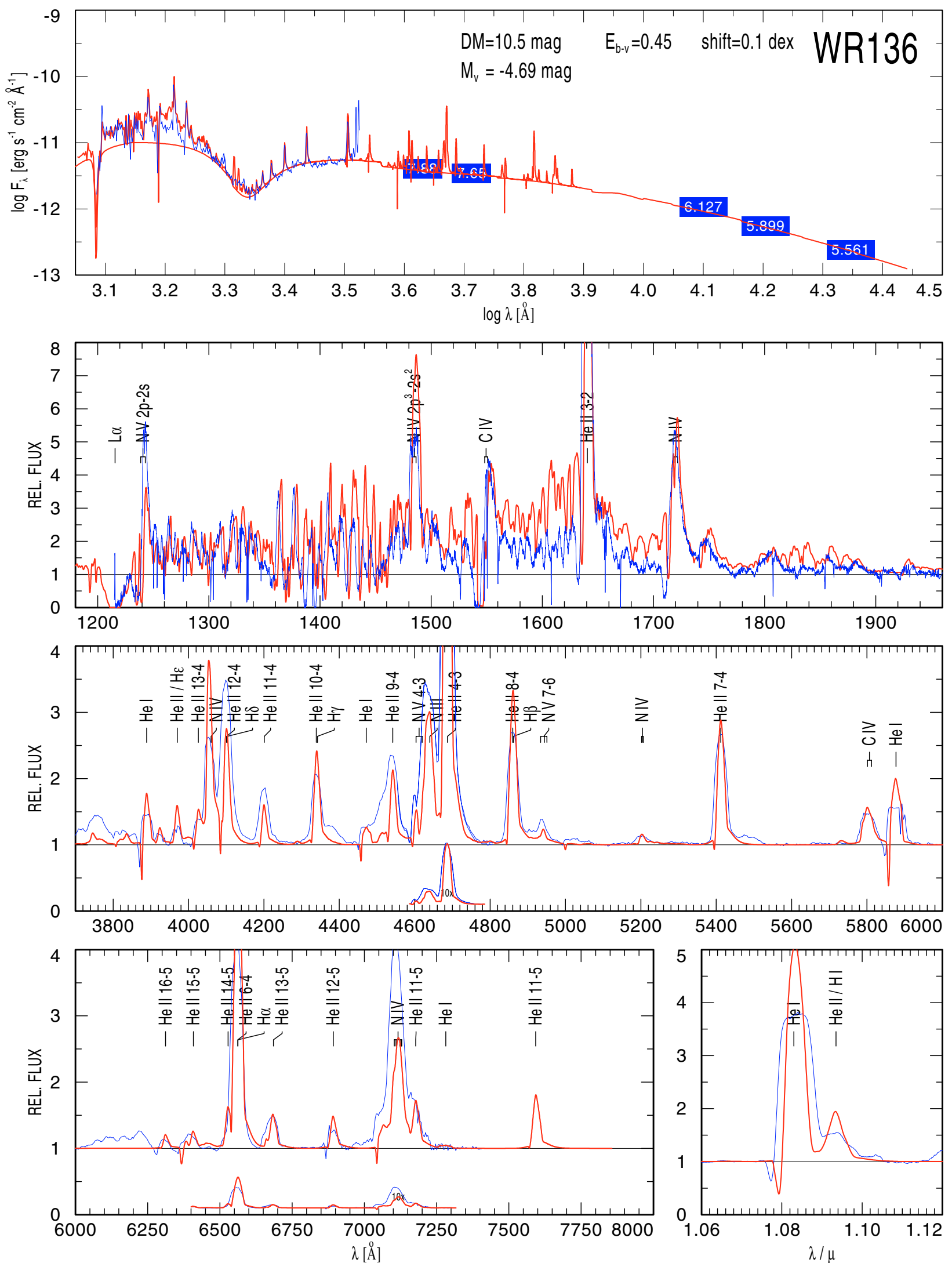

Fig. 68. Model: WNL 10-16, $T_{*}=71 \mathrm{kK}, \log \left(R_{\mathrm{t}} / R_{\odot}\right)=0.5$. 
W.-R. Hamann et al.: The Galactic WN stars, Online Material p 60

MODEL START 12/01/03 10:54:18 39811/0.9D/1000 L5.3 H20 N1.5\% C1E-4 Fe0.14\% D4 WNL-NODR 05-12 AFTER JOB NO.178
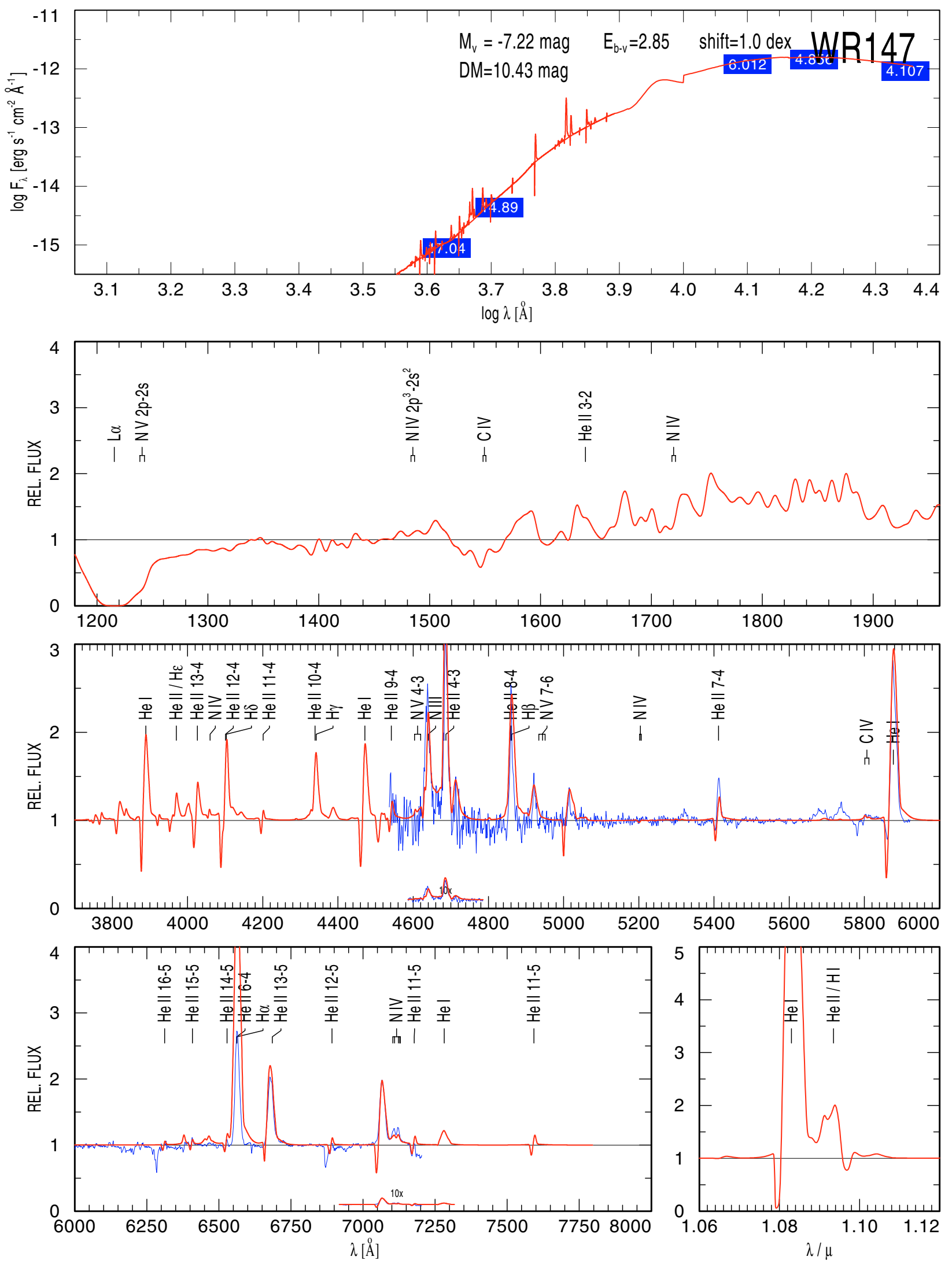

Fig. 69. Model: WNL 05-12, $T_{*}=40 \mathrm{kK}, \log \left(R_{\mathrm{t}} / R_{\odot}\right)=0.9$. 
W.-R. Hamann et al.: The Galactic WN stars, Online Material p 61

MODEL START 11/28/03 13:08:07 39811/1.30D/1000 L5.3 H20 N1.5\% C1E-4 Fe0.14\% D4 WNL-NODR 5-08 AFTER JOB N0.436
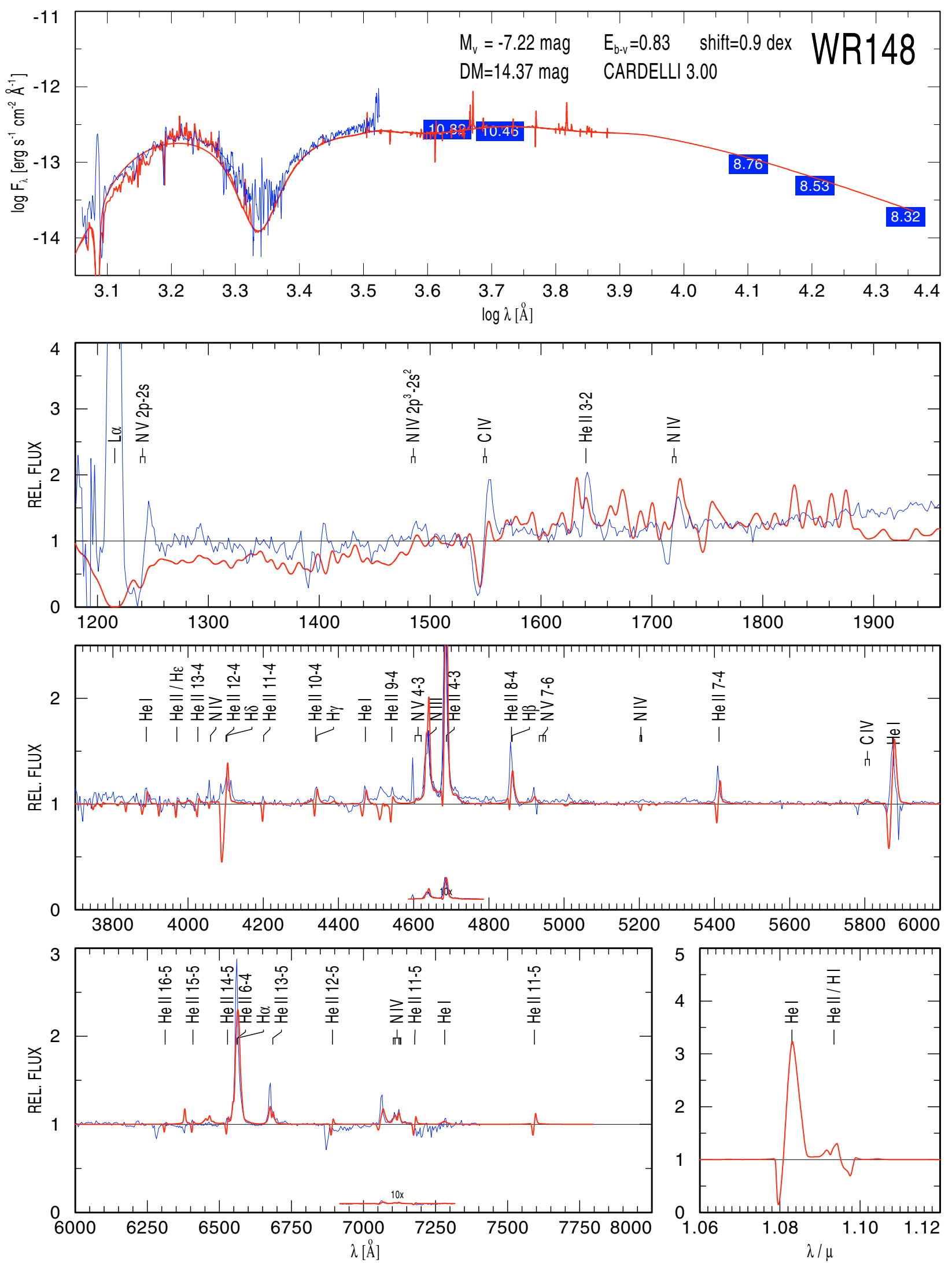

Fig. 70. Model: WNL 05-08, $T_{*}=40 \mathrm{kK}, \log \left(R_{\mathrm{t}} / R_{\odot}\right)=1.3$. 
W.-R. Hamann et al.: The Galactic WN stars, Online Material p 62

MODEL START 03/31/03 23:18:48 63096/0.7D/1600 L=5.3 N=1.5\% C=1E-4 Fe=1.4E-3 D4 WN-NODIEL 9-14 AFTER JOB NO.425
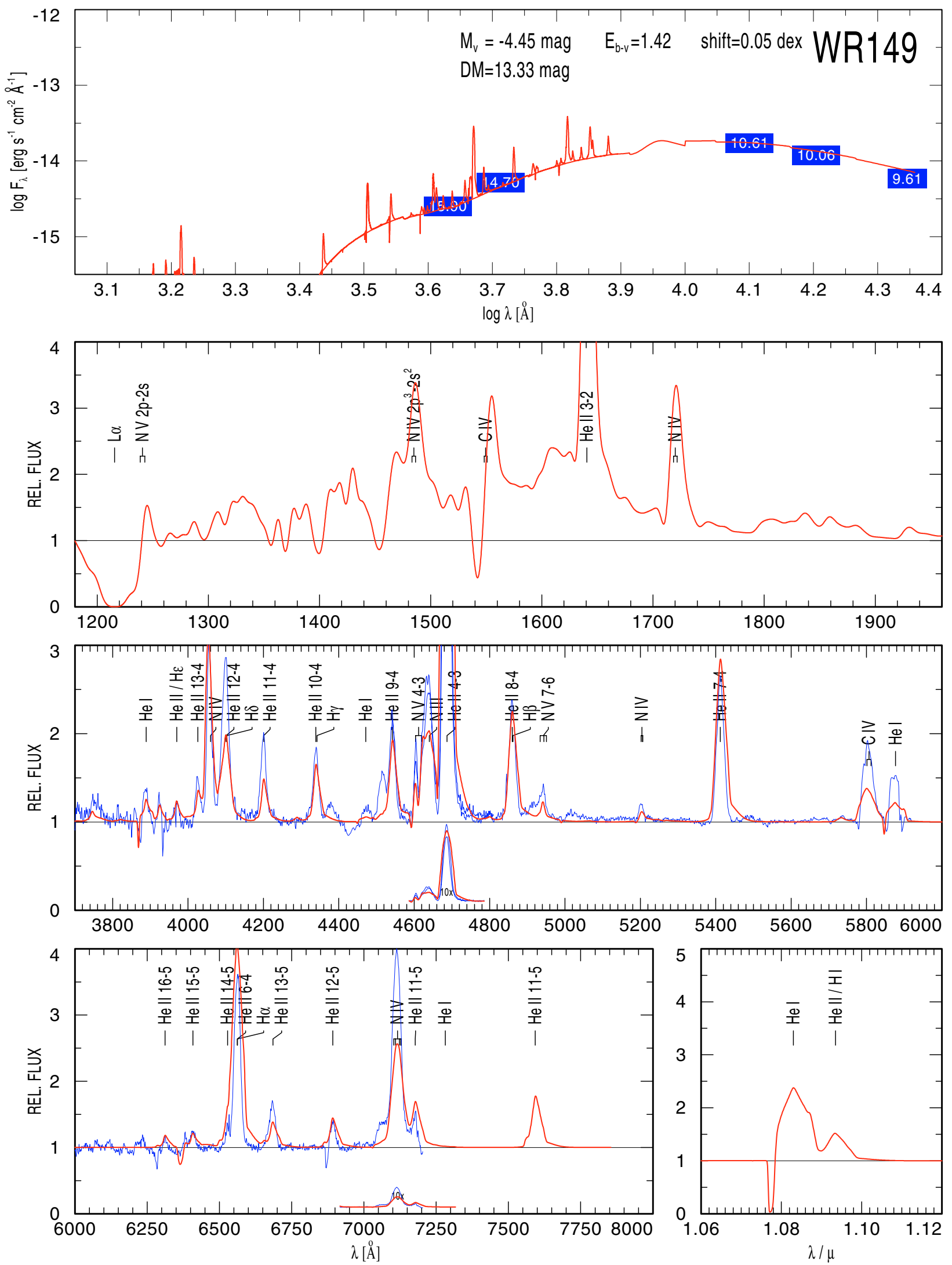

Fig. 71. Model: WNE 09-14, $T_{*}=63 \mathrm{kK}, \log \left(R_{\mathrm{t}} / R_{\odot}\right)=0.7$. 
W.-R. Hamann et al.: The Galactic WN stars, Online Material p 63

MODEL START 11/07/03 09:58:49 79433/1.1D/1000 L=5.3 H20 N1.5\% C1E-4 Fe0.14\% D4 WNL-NODR 11-10 AFTER JOB N0.414
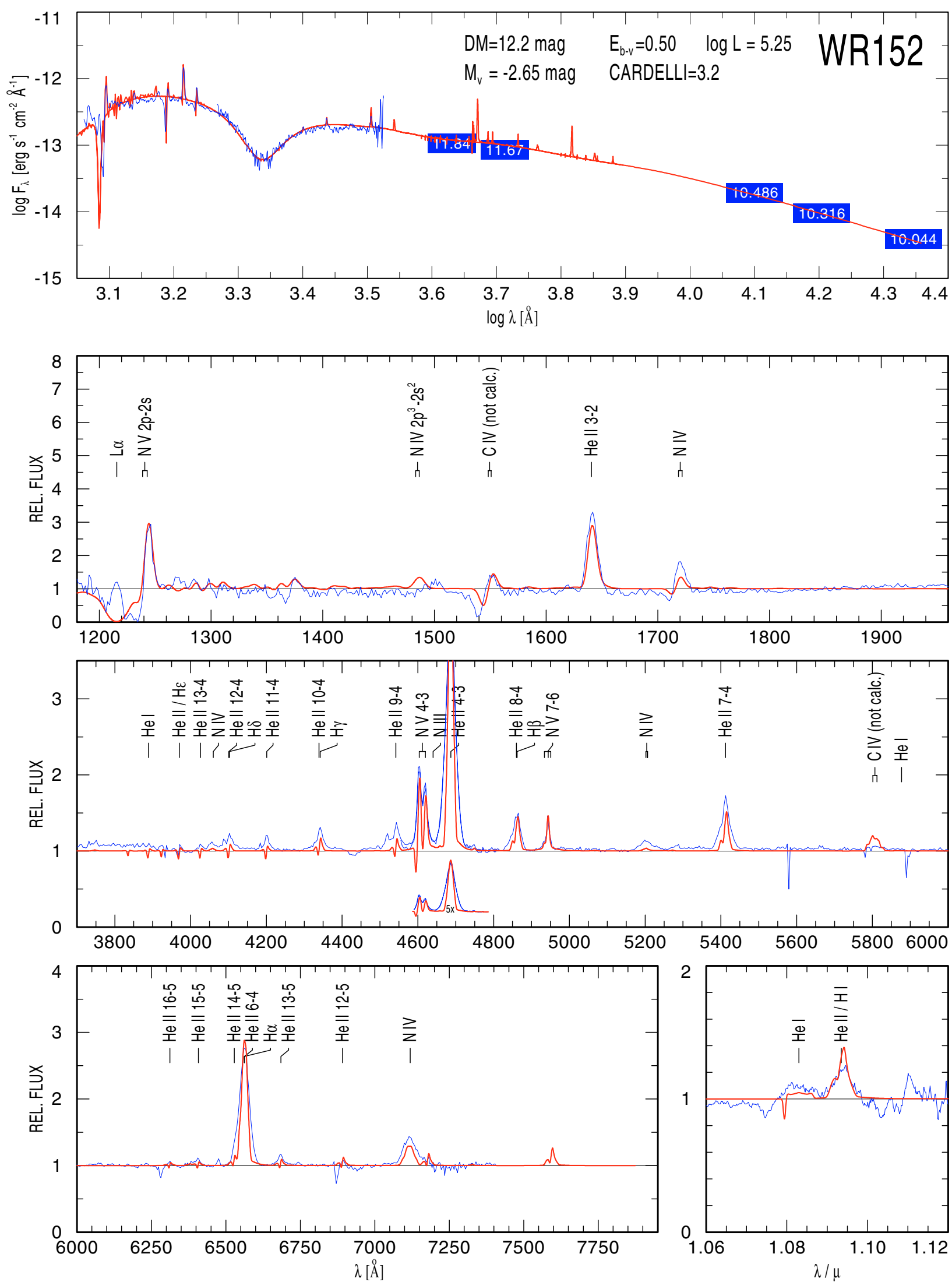

Fig. 72. Model: WNL 11-10, $T_{*}=79 \mathrm{kK}, \log \left(R_{\mathrm{t}} / R_{\odot}\right)=1.1$. 
W.-R. Hamann et al.: The Galactic WN stars, Online Material p 64

MODEL START 12/01/03 10:41:44 39811/1.1D/1000 L5.3 H20 N1.5\% C1E-4 Fe0.14\% D4 WNL-NODR 5-10 AFTER JOB N0.572
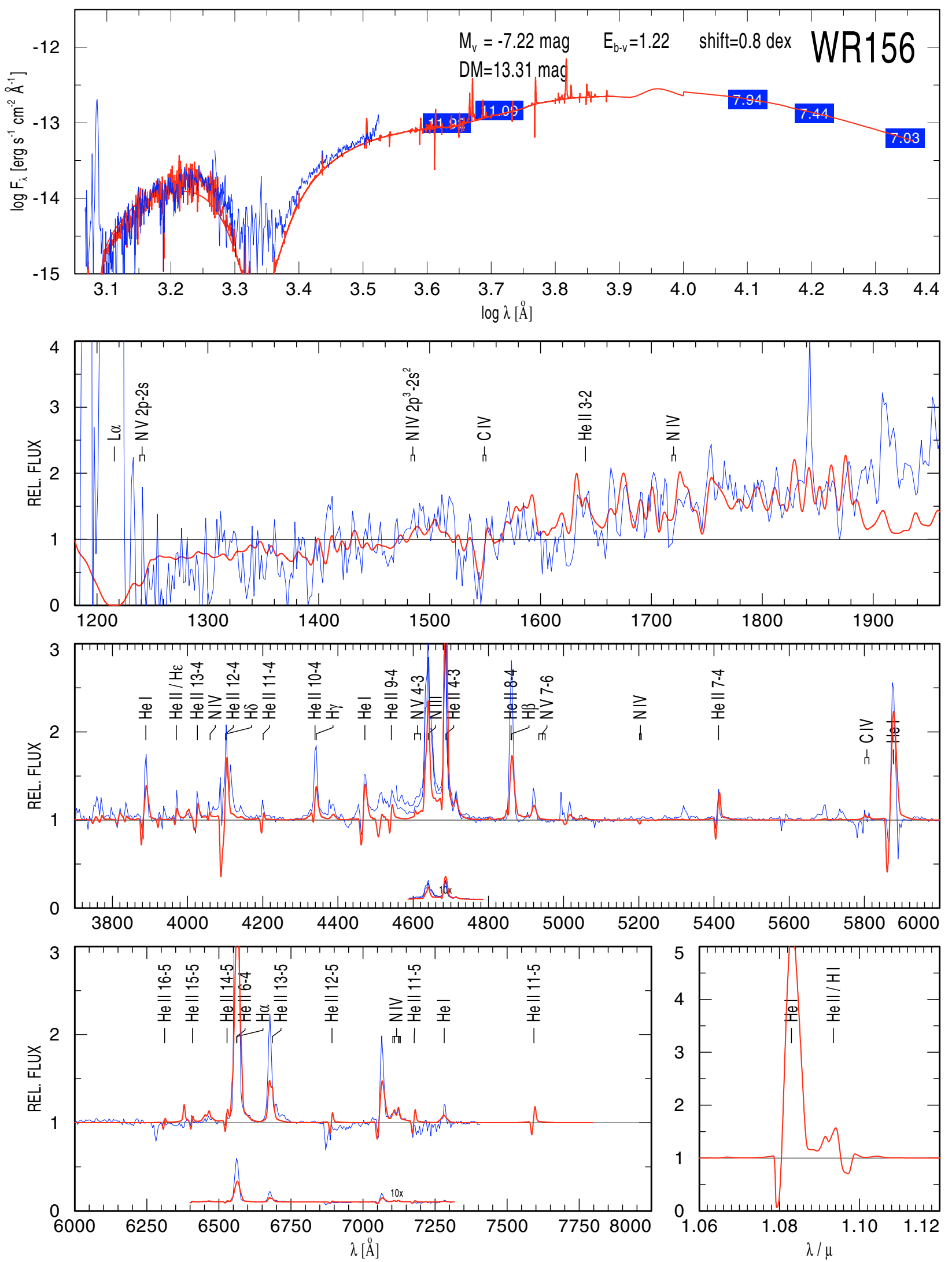

Fig. 73. Model: WNL 05-10, $T_{*}=40 \mathrm{kK}, \log \left(R_{\mathrm{t}} / R_{\odot}\right)=1.1$. 
W.-R. Hamann et al.: The Galactic WN stars, Online Material p 65

MODEL START 11/24/03 09:20:04 44668/1.2D/1000 L5.3 H20 N1.5\% C1E-4 Fe0.14\% D4 WNL-NODR 06-09 AFTER JOB N0.780
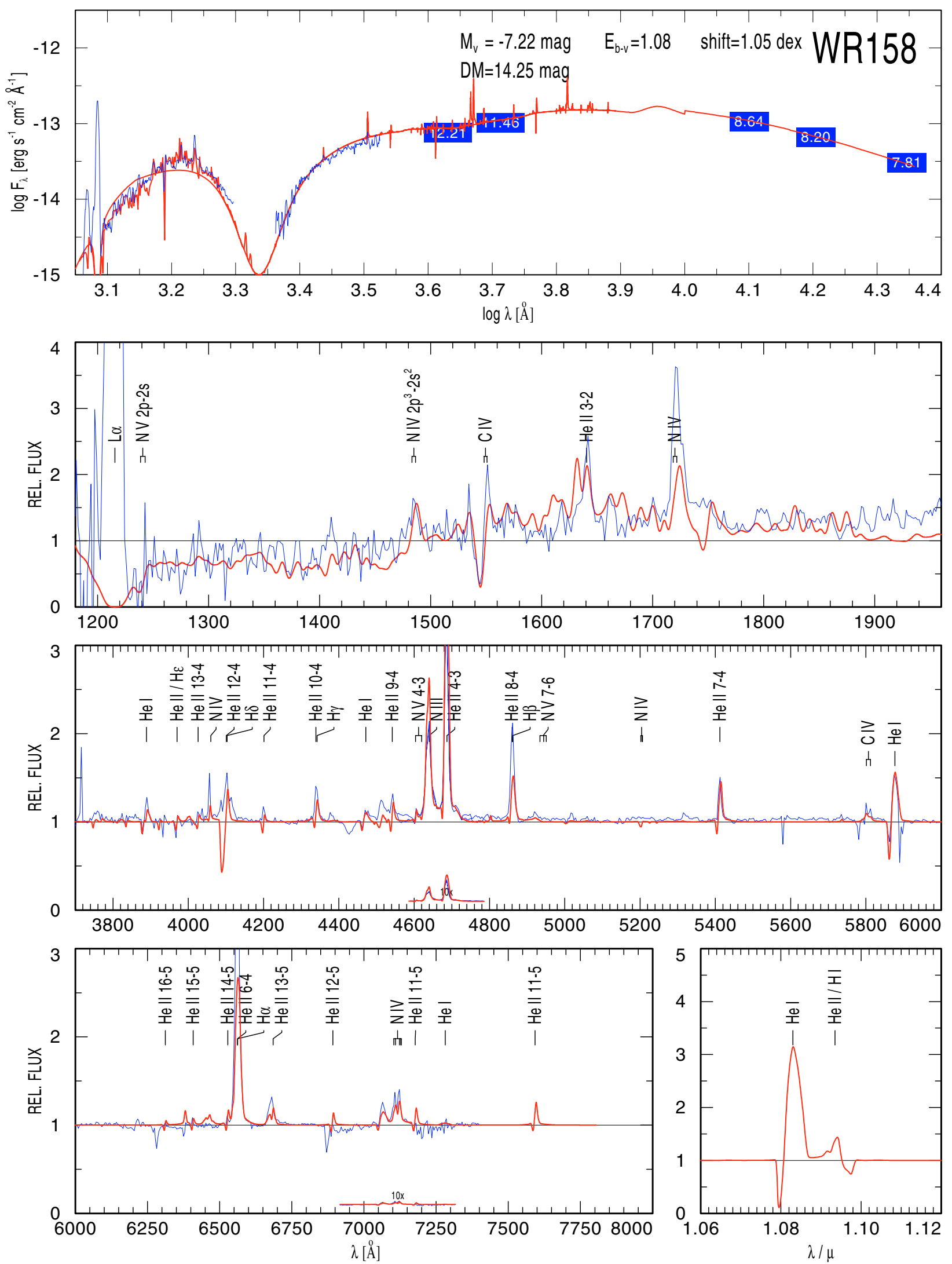

Fig. 74. Model: WNL 06-09, $T_{*}=45 \mathrm{kK}, \log \left(R_{\mathrm{t}} / R_{\odot}\right)=1.2$. 